\title{
A computational framework for polyconvex large strain elasticity
}

\author{
Javier Bonet ${ }^{1}$, Antonio J. Gil ${ }^{2}$, Rogelio Ortigosa \\ Zienkiewicz Centre for Computational Engineering, College of Engineering \\ Swansea University, Singleton Park, SA2 8PP, United Kingdom
}

\begin{abstract}
This paper presents a new computational formulation for large strain polyconvex elasticity. The formulation is based on using the deformation gradient (the fibre map), its adjoint (the area map) and its determinant (the volume map) as independent kinematic variables of a convex strain energy function. Compatibility relationships between these variables and the deformed geometry are enforced by means of a multi-field variational principle with additional constraints. This process allows the use of different approximation spaces for each variable. The paper also introduces conjugate stresses to these kinematic variables which can be used to define a generalised convex complementary energy function and a corresponding complementary energy principle of the Hellinger-Reissner type, where the new conjugate stresses are primary variables together with the deformed geometry. Both compressible and incompressible or nearly incompressible elastic models are considered. A key element to the developments presented in the paper is the definition of a new tensor cross product which facilitates the algebra associated with the adjoint of the deformation gradient. Two particular choices of interpolation spaces are considered to illustrate the formulation. The first is based on quadratic displacements, constant pressure discontinuous across element edges and linear discontinuous element stresses conjugate to the deformation gradient and its adjoint. The second choice involves linear displacement and continuous linear pressure stabilised using a Petrov-Galerkin technique.
\end{abstract}

Keywords: Large strain elasticity, polyconvex elasticity, mixed variational

\footnotetext{
${ }^{1}$ Corresponding author: j.bonet@swansea.ac.uk

${ }^{2}$ Corresponding author: a.j.gil@swansea.ac.uk
} 
principle, complementary energy variational principle, incompressible elasticity, finite elements

\section{Introduction}

Large strain elastic and inelastic analysis by finite elements or other computational techniques is now well established for many engineering applications [1-8]. Often elasticity is described by means of a hyperelastic model defined in terms a stored energy functional which depends on the deformation gradient of the mapping between initial and final configurations [1,9-17]. It has also been shown that for the model to be well defined in a mathematical sense, this dependency with respect to the deformation gradient has to satisfy certain convexity criteria $[1,12,13]$. The most well-established of these criteria is the concept of polyconvexity [14-20] whereby the strain energy function must be expressed as a convex function of the components of deformation gradient, its determinant and the components of its adjoint or cofactor. Numerous authors have previously incorporated this concept into computational models for both isotropic and non- isotropic materials for a variety of applications [21-26].

The standard approach consist of ensuring that the stored energy function satisfies the polyconvexity condition first but then proceed towards a computational solution by re-expressing the energy function in terms of the deformation gradient alone. More recently, a mixed formulation has been proposed in which the deformation gradient, its adjoint and its determinant are retained as fundamental problem variables by means of a $\mathrm{Hu}$-Washizu type of mixed variational principle [24, 27]. The resulting formulation opens up new interesting possibilities in terms of using various interpolation spaces for different variables [28-31], leading to enhanced type of formulations [24]. There is extensive literature in the field of mixed and enhanced formulations for large strain solid mechanics by numerous authors [32-43].

The present contribution aims to present a systematic framework for developing computational approaches for hyperelastic (or hyperelastic-plastic) polyconvex materials. Similarly to reference [24, 44, 45], the framework proposed is based on maintaining as independent variables the fundamental variables on which the strain energy is expressed as a convex function, namely, the deformation gradient, its adjoint and its determinant. These variables are, of course, not independent from each other but their relationships can 
be enforced by appropriate constraints in a multi-field variational principle leading to an extended $\mathrm{Hu}$-Washizu type of variational principle [1, 34]. In contrast with previous work, the paper proposes a novel algebra to deal with the additional kinematic variables based on the definition of the tensor cross product. This new notation not only greatly simplifies the formulation but it also provides new expressions for operators such as the tangent elasticity tensor which are useful from a computational and theoretical point of view.

The paper also introduces a set of stress variables work conjugate to the extended set of independent kinematic variables [46]. As a result of convexity with respect to this extended set, it is possible to define a complementary strain energy function which is convex with respect to this extended set of conjugate stresses. This definition makes it possible to introduce HellingerReissner [34, 39] type of mixed variational principles in the context of large strain elasticity, with a significantly reduced set of variables over a more traditional $\mathrm{Hu}$-Washizu type of functional.

In order to ensure that the new concepts proposed are presented in as clear a manner as possible, the paper only considers a Mooney-Rivlin type of constitutive model, both in the compressible and incompressible regime. This is the simplest model available which contains all the features of general polyconvex elasticity strain energy functions, that is a dependency with respect to the deformation gradient, its adjoint and its determinant in a convex manner. Extending the proposed formulation to more complex models is a simple algebraic exercise.

Note that it is not the primary aim of this paper to propose specific choices of interpolation spaces. Nevertheless, two examples of the use of the framework are provided. First, an application of the complementary energy functional with quadratic displacements and linear stresses is presented. This is followed by a model for incompressible elasticity using stabilised linear tetrahedral elements.

The paper is organised as follows. Section 2 introduces the novel tensor cross product notation in the context of large strain deformation. This definition is used to re-express the adjoint of the deformation gradient and its directional derivatives in a novel, simple and convenient manner. Section 3 reviews the definition of polyconvex elastic strain energy functions and defines a new set of stresses conjugate to the main kinematic variables. The relationships between these stresses and standard stress tensors such as the Piola-Kirchhoff stresses and Cauchy stresses are provided. The section also derives complementary strain energy functions in terms of the new conju- 
gate stresses. The fourth order elasticity tensors are derived in this section taking advantage of the new tensor cross product notation, which greatly simplifies the algebra involved and leads to interesting insights into the consequences of convexity. Both compressible and nearly incompressible cases are discussed in the context of Mooney-Rivlin models, although the extension to more general strain energy functions is straight forward. Section 4 presents a range of multi-field variational principles based on the extended set of kinematic and stress variables. This includes displacement based principles, Hu-Washizu type of mixed principles including additional kinematic and stress variables and Hellinger-Reissner principles including geometry and stress variables. Section 5 illustrates the use of the above principles in the context of finite element interpolations. The resulting discretised equations are presented for two particular choices of interpolation spaces. A number of benchmark examples are used in Section 6 in order to demonstrate the validity and convergence characteristics of the formulation proposed. Finally Section 7 provides some concluding remarks and a summary of the key contributions of this paper.

\section{Definitions and notation}

\subsection{Motion and deformation}

Consider the 3-dimensional deformation of an elastic body from its initial configuration occupying a volume $V$, of boundary $\partial V$, into a final configuration at volume $v$, of boundary $\partial v$ (see Figure 1 ). The standard notation and definitions for the deformation gradient and its determinant are used:

$$
\boldsymbol{F}=\frac{\partial \boldsymbol{x}}{\partial \boldsymbol{X}}=\nabla_{0} \boldsymbol{x} ; \quad J=\operatorname{det} \boldsymbol{F}=\frac{d v}{d V}
$$

where $\boldsymbol{x}$ represents the current position of a particle originally at $\boldsymbol{X}$ and $\boldsymbol{\nabla}_{0}$ denotes the gradient with respect to material coordinates. Virtual or incremental variations of $\boldsymbol{x}$ will be denoted $\delta \boldsymbol{v}$ and $\boldsymbol{u}$, respectively. It will be assumed that $\boldsymbol{x}, \delta \boldsymbol{v}$ and $\boldsymbol{u}$ satisfy appropriate displacement based boundary conditions in $\partial_{u} V$. Additionally, the body is under the action of certain body forces per unit undeformed volume $\boldsymbol{f}_{0}$ and traction per unit undeformed area $\boldsymbol{t}_{0}$ in $\partial_{t} V$, where $\partial_{t} V \cup \partial_{u} V=\partial V$ and $\partial_{t} V \cap \partial_{u} V=\emptyset$.

The element area vector is mapped from initial $d \boldsymbol{A}$ to final $d \boldsymbol{a}$ configuration by means of the two-point tensor $\boldsymbol{H}$, which is related to the deformation 


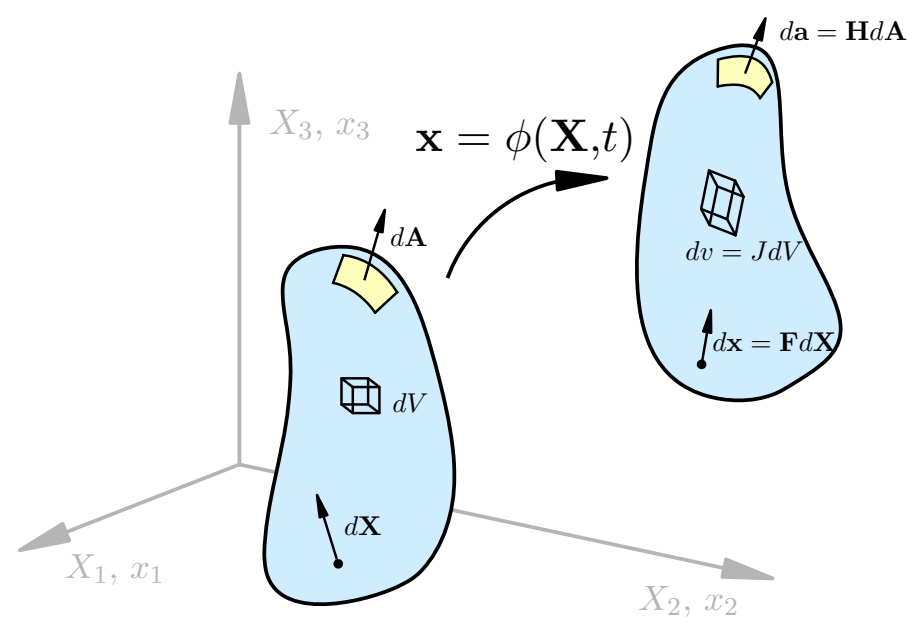

Figure 1: Deformation mapping of a continuum and associated kinematics magnitudes: $\boldsymbol{F}, \boldsymbol{H}, J$.

gradient via Nanson's rule [1]:

$$
d \boldsymbol{a}=\boldsymbol{H} d \boldsymbol{A} ; \quad \boldsymbol{H}=J \boldsymbol{F}^{-T} .
$$

Clearly, the components of this tensor are the order 2 minors of the deformation gradient and it is often referred to as the co-factor or adjoint tensor. This tensor and its derivatives will feature heavily in the formulation that follows as it is a key variable for polyconvex elastic models. Its evaluation and, more importantly, the evaluation of its derivatives using equation (2) is not ideal, and a more convenient formula can be derived for 3-dimensional applications. This relies on the definition of the tensor cross product given in the next section.

\subsection{Tensor cross product}

One of the key elements of the framework proposed is the extension of the standard vector cross product to define the cross product between second order tensors and between tensors and vectors. For instance, the left cross product of a vector $\boldsymbol{v}$ and a second order tensor $\boldsymbol{A}$ to give a second order tensor denoted $\boldsymbol{v} \times \boldsymbol{A}$ is defined so that when applied to a general vector $\boldsymbol{w}$ gives:

$$
(\boldsymbol{v} \times \boldsymbol{A}) \boldsymbol{w}=\boldsymbol{v} \times(\boldsymbol{A} \boldsymbol{w}) ; \quad(\boldsymbol{v} \times \boldsymbol{A})_{i j}=\mathcal{E}_{i k l} v_{k} A_{l j}
$$


where $\mathcal{E}_{i k l}$ denote the standard third order alternating tensor components, repeated indices indicate summation and $\times$ is the standard vector cross product. The effect of the above operation is to replace the columns of $\boldsymbol{A}$ by the cross products between $\boldsymbol{v}$ and the original columns of $\boldsymbol{A}$. Similarly, the right cross product of a second order tensor $\boldsymbol{A}$ by a vector $\boldsymbol{v}$ to give a second order tensor denoted $\boldsymbol{A} \times \boldsymbol{v}$ is defined so that for every vector $\boldsymbol{w}$ the following relationship applies:

$$
(\boldsymbol{A} \times \boldsymbol{v}) \boldsymbol{w}=\boldsymbol{A}(\boldsymbol{v} \times \boldsymbol{w}) ; \quad(\boldsymbol{A} \times \boldsymbol{v})_{i j}=\mathcal{E}_{j k l} A_{i k} v_{l} .
$$

The effect is now to replace the rows of $\boldsymbol{A}$ by the cross products of its original rows by $\boldsymbol{v}$.

Finally, the cross product of two second order tensors $\boldsymbol{A}$ and $\boldsymbol{B}$ to give a new second order tensor denoted $\boldsymbol{A} \times \boldsymbol{B}$ is defined so that for any arbitrary vectors $\boldsymbol{v}$ and $\boldsymbol{w}$ gives:

$$
\boldsymbol{v} \cdot(\boldsymbol{A} \times \boldsymbol{B}) \boldsymbol{w}=(\boldsymbol{v} \times \boldsymbol{A}):(\boldsymbol{B} \times \boldsymbol{w}) ; \quad(\boldsymbol{A} \times \boldsymbol{B})_{i j}=\mathcal{E}_{i k l} \mathcal{E}_{j m n} A_{k m} B_{l n} .
$$

In the framework developed in this paper the tensor cross product will be mostly applied between two-point tensors. For this purpose the above definition can be readily particularised to second order two-point tensors or material tensors as,

$$
(\boldsymbol{A} \times \boldsymbol{B})_{i I}=\mathcal{E}_{i j k} \mathcal{E}_{I J K} A_{j J} B_{k K} ; \quad(\boldsymbol{A} \times \boldsymbol{B})_{I J}=\mathcal{E}_{I K L} \mathcal{E}_{J M N} A_{K M} B_{L N}
$$

Box 1 shows the practical evaluation of these products.

Remark 1: It is easy to show using simply algebraic manipulations based on the permutation properties of $\mathcal{E}$ or the fact that $\mathcal{E}_{i j k} \mathcal{E}_{k l n}=\delta_{i l} \delta_{j n}-\delta_{i n} \delta_{j l}$, that the above tensor cross products satisfy the following properties (note that $\boldsymbol{v}$, $\boldsymbol{v}_{1}, \boldsymbol{v}_{2}, \boldsymbol{w}, \boldsymbol{w}_{1}$ and $\boldsymbol{w}_{2}$ denote arbitrary vectors and $\boldsymbol{A}, \boldsymbol{A}_{1}, \boldsymbol{A}_{2}, \boldsymbol{B}, \boldsymbol{B}_{1}, \boldsymbol{B}_{2}$ and $\boldsymbol{C}$ are second order tensors):

$$
\begin{gathered}
\boldsymbol{A} \times \boldsymbol{B}=\boldsymbol{B} \times \boldsymbol{A} \\
(\boldsymbol{A} \times \boldsymbol{B})^{T}=\boldsymbol{A}^{T} \times \boldsymbol{B}^{T} \\
\boldsymbol{A} \times\left(\boldsymbol{B}_{1}+\boldsymbol{B}_{2}\right)=\boldsymbol{A} \times \boldsymbol{B}_{1}+\boldsymbol{A} \times \boldsymbol{B}_{2} \\
\alpha(\boldsymbol{A} \times \boldsymbol{B})=(\alpha \boldsymbol{A}) \times \boldsymbol{B}=\boldsymbol{A} \times(\alpha \boldsymbol{B})
\end{gathered}
$$




$$
\begin{gathered}
(\boldsymbol{A} \times \boldsymbol{B}): \boldsymbol{C}=(\boldsymbol{B} \times \boldsymbol{C}): \boldsymbol{A}=(\boldsymbol{A} \times \boldsymbol{C}): \boldsymbol{B} \\
\boldsymbol{A} \times \boldsymbol{I}=(\operatorname{tr} \boldsymbol{A}) \boldsymbol{I}-\boldsymbol{A}^{T} \\
\boldsymbol{I} \times \boldsymbol{I}=2 \boldsymbol{I} \\
(\boldsymbol{A} \times \boldsymbol{A}): \boldsymbol{A}=6 \operatorname{det} \boldsymbol{A} \\
\operatorname{Cof} \boldsymbol{A}=\frac{1}{2} \boldsymbol{A} \times \boldsymbol{A} \\
\left(\boldsymbol{v}_{1} \otimes \boldsymbol{v}_{2}\right) \times\left(\boldsymbol{w}_{1} \otimes \boldsymbol{w}_{2}\right)=\left(\boldsymbol{v}_{1} \times \boldsymbol{w}_{1}\right) \otimes\left(\boldsymbol{v}_{2} \times \boldsymbol{w}_{2}\right) \\
\boldsymbol{v} \times(\boldsymbol{A} \times \boldsymbol{w})=(\boldsymbol{v} \times \boldsymbol{A}) \times \boldsymbol{w}=\boldsymbol{v} \times \boldsymbol{A} \times \boldsymbol{w} \\
\boldsymbol{A} \times(\boldsymbol{v} \otimes \boldsymbol{w})=-\boldsymbol{v} \times \boldsymbol{A} \times \boldsymbol{w} \\
(\boldsymbol{A} \times \boldsymbol{B})(\boldsymbol{v} \times \boldsymbol{w})=(\boldsymbol{A} \boldsymbol{v}) \times(\boldsymbol{B} \boldsymbol{w})+(\boldsymbol{B} \boldsymbol{v}) \times(\boldsymbol{A} \boldsymbol{w}) \\
\left(\boldsymbol{A}_{1} \times \boldsymbol{A}_{2}\right)\left(\boldsymbol{B}_{1} \times \boldsymbol{B}_{2}\right)=\left(\boldsymbol{A}_{1} \boldsymbol{B}_{1} \times \boldsymbol{A}_{2} \boldsymbol{B}_{2}\right)+\left(\boldsymbol{A}_{1} \boldsymbol{B}_{2} \times \boldsymbol{A}_{2} \boldsymbol{B}_{1}\right) \\
\boldsymbol{A}_{1} \boldsymbol{B} \times \boldsymbol{A}_{2} \boldsymbol{B}=\left(\boldsymbol{A}_{1} \times \boldsymbol{A}_{2}\right)(\mathrm{Cof} \boldsymbol{B})
\end{gathered}
$$




\section{Box 1. Enumeration of tensor cross products:}

$$
\begin{aligned}
{[\boldsymbol{v} \times \boldsymbol{A}] } & =\left[\begin{array}{lll}
v_{y} A_{z x}-v_{z} A_{y x} & v_{y} A_{z y}-v_{z} A_{y y} & v_{y} A_{z z}-v_{z} A_{y z} \\
v_{z} A_{x x}-v_{x} A_{z x} & v_{z} A_{x y}-v_{x} A_{z y} & v_{z} A_{x z}-v_{x} A_{z z} \\
v_{x} A_{y x}-v_{y} A_{x x} & v_{x} A_{y y}-v_{y} A_{x y} & v_{x} A_{y z}-v_{y} A_{x z}
\end{array}\right] \\
{[\boldsymbol{A} \times \boldsymbol{w}] } & =\left[\begin{array}{lll}
A_{x y} v_{z}-A_{x z} v_{y} & A_{x z} v_{x}-A_{x x} v_{z} & A_{x x} v_{y}-A_{x y} v_{x} \\
A_{y y} v_{z}-A_{y z} v_{x} & A_{y z} v_{x}-A_{y x} v_{z} & A_{y x} v_{y}-A_{y y} v_{x} \\
A_{y x} v_{z}-A_{x x} v_{y} & A_{y y} v_{x}-A_{x y} v_{z} & A_{y z} v_{y}-A_{x z} v_{x}
\end{array}\right] \\
{[\boldsymbol{A} \times \boldsymbol{B}] } & =\left[\begin{array}{ll}
{[\boldsymbol{A} \times \boldsymbol{B}]_{x x}} & {[\boldsymbol{A} \times \boldsymbol{B}]_{x y}[\boldsymbol{A} \times \boldsymbol{B}]_{x z}} \\
{[\boldsymbol{A} \times \boldsymbol{B}]_{y x}} & {[\boldsymbol{A} \times \boldsymbol{B}]_{y y}[\boldsymbol{A} \times \boldsymbol{B}]_{y z}} \\
{[\boldsymbol{A} \times \boldsymbol{B}]_{z x}} & {[\boldsymbol{A} \times \boldsymbol{B}]_{z y}[\boldsymbol{A} \times \boldsymbol{B}]_{z z}}
\end{array}\right] \\
{[\boldsymbol{A} \times \boldsymbol{B}]_{x x} } & =A_{y y} B_{z z}-A_{y z} B_{z y}+A_{z z} B_{y y}-A_{z y} B_{y z} \\
{[\boldsymbol{A} \times \boldsymbol{B}]_{x y} } & =A_{y z} B_{z x}-A_{y x} B_{z z}+A_{z x} B_{y z}-A_{z z} B_{y x} \\
{[\boldsymbol{A} \times \boldsymbol{B}]_{x z} } & =A_{y x} B_{z y}-A_{y y} B_{z x}+A_{z y} B_{y x}-A_{z x} B_{y y} \\
{[\boldsymbol{A} \times \boldsymbol{B}]_{y x} } & =A_{x z} B_{z y}-A_{x y} B_{z z}+A_{z y} B_{x z}-A_{z z} B_{x y} \\
{[\boldsymbol{A} \times \boldsymbol{B}]_{y y} } & =A_{z z} B_{x x}-A_{z x} B_{x z}+A_{x x} B_{z z}-A_{x z} B_{z x} \\
{[\boldsymbol{A} \times \boldsymbol{B}]_{y z} } & =A_{z x} B_{x y}-A_{z y} B_{x x}+A_{x y} B_{z x}-A_{x x} B_{z y} \\
{[\boldsymbol{A} \times \boldsymbol{B}]_{z x} } & =A_{x y} B_{y z}-A_{x z} B_{y y}+A_{y z} B_{x y}-A_{y y} B_{x z} \\
{[\boldsymbol{A} \times \boldsymbol{B}]_{z y} } & =A_{x z} B_{y x}-A_{x x} B_{y z}+A_{y x} B_{x z}-A_{y z} B_{x x} \\
{[\boldsymbol{A} \times \boldsymbol{B}]_{z z} } & =A_{x x} B_{y y}-A_{x y} B_{y x}+A_{y y} B_{x x}-A_{y x} B_{x y}
\end{aligned}
$$

\subsection{Alternative expressions for the area and volume maps}

Using the ninth property in Remark 1, equation (15), it is now possible to express the area map tensor $\boldsymbol{H}$ as:

$$
\boldsymbol{H}=\frac{1}{2} \boldsymbol{F} \times \boldsymbol{F}
$$

Moreover, the first and second directional derivatives of $\boldsymbol{H}$ with respect 
to geometry changes are now easily evaluated as:

$$
\begin{gathered}
D \boldsymbol{H}[\delta \boldsymbol{v}]=\boldsymbol{F} \times D \boldsymbol{F}[\delta \boldsymbol{v}]=\boldsymbol{F} \times \boldsymbol{\nabla}_{0} \delta \boldsymbol{v} \\
D^{2} \boldsymbol{H}[\delta \boldsymbol{v}, \boldsymbol{u}]=D \boldsymbol{F}[\boldsymbol{u}] \times D \boldsymbol{F}[\delta \boldsymbol{v}]=\boldsymbol{\nabla}_{0} \delta \boldsymbol{v} \times \boldsymbol{\nabla}_{0} \boldsymbol{u}
\end{gathered}
$$

Similarly, the derivatives of the volume ratio $J$ are easily expressed with the help of (14) and (11) and the definition of $\boldsymbol{H}$ given by equation (22):

$$
D J[\delta \boldsymbol{v}]=\boldsymbol{H}: \boldsymbol{\nabla}_{0} \delta \boldsymbol{v} ; \quad D^{2} \boldsymbol{J}[\delta \boldsymbol{v}, \boldsymbol{u}]=\boldsymbol{F}:\left(\boldsymbol{\nabla}_{0} \delta \boldsymbol{v} \times \boldsymbol{\nabla}_{0} \boldsymbol{u}\right)
$$

The above formulas simplify the manipulation of the derivatives of $\boldsymbol{H}$ and $J$ by avoiding differentiating the inverse of the deformation gradient. They will be key to the development of the framework presented below.

It is also possible to derive alternative expressions for both $\boldsymbol{H}$ and $J$. For instance, combining equation (22) with equation (1) and noting that the derivatives of $\boldsymbol{F}$ are second derivatives of $\boldsymbol{x}$ and therefore symmetric, gives, after simple use of the product rule:

$$
\boldsymbol{H}=\frac{1}{2} \mathrm{CURL}(\boldsymbol{x} \times \boldsymbol{F})
$$

where the material CURL of a second order two point tensor is defined in the usual fashion by:

$$
(\mathrm{CURL} \boldsymbol{A})_{i I}=\mathcal{E}_{I J K} \frac{\partial A_{i K}}{\partial X_{J}}
$$

It is clear from equation (26) that the material divergence of $\boldsymbol{H}$ vanishes, as does the material CURL of $\boldsymbol{F}$, that is:

$$
\mathrm{DIV} \boldsymbol{H}=\mathbf{0} ; \quad \mathrm{CURL} \boldsymbol{F}=\mathbf{0}
$$

where the material divergence is defined by the contraction:

$$
(\operatorname{DIV} \boldsymbol{A})_{i}=\frac{\partial A_{i I}}{\partial X_{I}}
$$

Combining equations (1) and (2b) an alternative equation for $J$ emerges as:

$$
J=\frac{1}{3} \boldsymbol{H}: \nabla_{0} \boldsymbol{x}=\frac{1}{3} \operatorname{DIV}\left(\boldsymbol{H}^{T} \boldsymbol{x}\right) .
$$




\section{Polyconvex elasticity}

\subsection{The strain energy}

Polyconvexity is now well accepted as a fundamental mathematical requirement that must be satisfied by admissible strain energy functions used to describe elastic materials in the large strain regime. Essentially, the strain energy $\Psi$ per unit undeformed volume must be a function of the deformation gradient $\boldsymbol{F}$ via a convex multi-valued function $W$ as:

$$
\Psi\left(\boldsymbol{\nabla}_{0} \boldsymbol{x}\right)=W(\boldsymbol{F}, \boldsymbol{H}, J)
$$

where $W$ is convex with respect to its 19 variables, namely, $J$ and the $3 \times 3$ components of $\boldsymbol{F}$ and $\boldsymbol{H}$. Moreover, invariance with respect to rotations in the material configuration implies that $W$ must be independent of the rotational components of $\boldsymbol{F}$ and $\boldsymbol{H}$. This is typically achieved by ensuring that $W$ depends on $\boldsymbol{F}$ and $\boldsymbol{H}$ via the symmetric tensors $\boldsymbol{C}=\boldsymbol{F}^{T} \boldsymbol{F}$ and $\boldsymbol{G}=\boldsymbol{H}^{T} \boldsymbol{H}$, respectively. In fact, for isotropic materials, this dependency can be further simplified through the use of invariants and the observation that:

$$
I_{\boldsymbol{C}}=\boldsymbol{F}: \boldsymbol{F} ; \quad I I_{\boldsymbol{C}}=\boldsymbol{H}: \boldsymbol{H} ; \quad I I I_{\boldsymbol{C}}=J^{2} .
$$

For example, a general compressible Mooney-Rivlin material can be described by an energy function of the type:

$$
W_{M R}=\alpha \boldsymbol{F}: \boldsymbol{F}+\beta \boldsymbol{H}: \boldsymbol{H}+f(J)
$$

where $\alpha$ and $\beta$ are positive material parameters and $f$ denotes a convex function of $J$. It is clear therefore that $W_{M R}$ is convex with respect to all of its variables. The condition of vanishing energy at the initial reference configuration can be established by ensuring that $f(1)=-(3 \alpha+3 \beta)$ or by adding an appropriate constant to $W_{M R}$. Doing this, however, has no practical effect on the resulting formulation as this will be driven by derivatives of the strain energy. Appropriate values for $\alpha$ and $\beta$ and suitable functions $f$ will be found in the sections below.

\subsection{Conjugate stresses and the first Piola-kirchhoff tensor}

The three strain measures $\boldsymbol{F}, \boldsymbol{H}$, and $J$ have work conjugate stresses $\boldsymbol{\Sigma}_{\boldsymbol{F}}$, $\boldsymbol{\Sigma}_{\boldsymbol{H}}$, and $\Sigma_{J}$ defined by:

$$
\boldsymbol{\Sigma}_{\boldsymbol{F}}(\boldsymbol{F}, \boldsymbol{H}, J)=\frac{\partial W}{\partial \boldsymbol{F}} ; \quad \boldsymbol{\Sigma}_{\boldsymbol{H}}(\boldsymbol{F}, \boldsymbol{H}, J)=\frac{\partial W}{\partial \boldsymbol{H}} ; \quad \Sigma_{J}(\boldsymbol{F}, \boldsymbol{H}, J)=\frac{\partial W}{\partial J} .
$$


For instance, for the case of a Mooney-Rivlin material, these stresses become:

$$
\boldsymbol{\Sigma}_{\boldsymbol{F}}=2 \alpha \boldsymbol{F} ; \quad \boldsymbol{\Sigma}_{\boldsymbol{H}}=2 \beta \boldsymbol{F} ; \quad \Sigma_{J}=f^{\prime}(J) .
$$

The set of conjugate stresses defined in equation (34) enables the directional derivative of the stain energy to be expressed as:

$$
D W[\delta \boldsymbol{F}, \delta \boldsymbol{H}, \delta J]=\boldsymbol{\Sigma}_{\boldsymbol{F}}: \delta \boldsymbol{F}+\boldsymbol{\Sigma}_{\boldsymbol{H}}: \delta \boldsymbol{H}+\Sigma_{J} \delta J .
$$

In order to develop a relationship between these conjugate stresses and the more standard first Piola-Kirchhoff, recall first that the first Piola-Kirchoff tensor is defined by the equation:

$$
D \Psi[\delta \boldsymbol{v}]=\boldsymbol{P}: \nabla_{0} \delta \boldsymbol{v} ; \quad \boldsymbol{P}=\left.\frac{\partial \Psi(\boldsymbol{F})}{\partial \boldsymbol{F}}\right|_{\boldsymbol{F}=\nabla_{0} \boldsymbol{x}} .
$$

With the help of the last two equations, the chain rule and equations (23) and (25a) it is possible to express the virtual internal work as:

$$
\begin{aligned}
\boldsymbol{P}: \boldsymbol{\nabla}_{0} \delta \boldsymbol{v} & =D \Psi[\delta \boldsymbol{v}] \\
& =D W[D \boldsymbol{F}[\delta \boldsymbol{v}], D \boldsymbol{H}[\delta \boldsymbol{v}], D J[\delta \boldsymbol{v}]] \\
& =\boldsymbol{\Sigma}_{\boldsymbol{F}}: D \boldsymbol{F}[\delta \boldsymbol{v}]+\boldsymbol{\Sigma}_{\boldsymbol{H}}: D \boldsymbol{H}[\delta \boldsymbol{v}]+\Sigma_{J} D J[\delta \boldsymbol{v}] \\
& =\boldsymbol{\Sigma}_{\boldsymbol{F}}: \boldsymbol{\nabla}_{0} \delta \boldsymbol{v}+\boldsymbol{\Sigma}_{\boldsymbol{H}}:\left(\boldsymbol{F} \times \boldsymbol{\nabla}_{0} \delta \boldsymbol{v}\right)+\Sigma_{J}\left(\boldsymbol{H}: \boldsymbol{\nabla}_{0} \delta \boldsymbol{v}\right) \\
& =\left(\boldsymbol{\Sigma}_{\boldsymbol{F}}+\boldsymbol{\Sigma}_{\boldsymbol{H}} \times \boldsymbol{F}+\Sigma_{J} \boldsymbol{H}\right): \boldsymbol{\nabla}_{0} \delta \boldsymbol{v}
\end{aligned}
$$

which leads to the evaluation to the first Piola-Kirchhoff tensor as:

$$
\boldsymbol{P}=\boldsymbol{\Sigma}_{\boldsymbol{F}}+\boldsymbol{\Sigma}_{\boldsymbol{H}} \times \boldsymbol{F}+\Sigma_{J} \boldsymbol{H}
$$

For instance, for the simple compressible Mooney-Rivlin material defined above, this expression becomes:

$$
\boldsymbol{P}=2 \alpha \boldsymbol{F}+2 \beta \boldsymbol{H} \times \boldsymbol{F}+f^{\prime}(J) \boldsymbol{H} .
$$

The condition of a stress-free initial configuration, where $\boldsymbol{F}=\boldsymbol{H}=\boldsymbol{I}$ and $J=1$, together with property (13) of the tensor cross product leads to the following constraint on the material parameters $\alpha, \beta$ and the function $f(J)$ :

$$
2 \alpha+4 \beta+f^{\prime}(1)=0 .
$$




\subsection{Complementary energy}

The convexity of the function $W(\boldsymbol{F}, \boldsymbol{H}, J)$ with respect to its variables ensures that the relationships between $\{\boldsymbol{F}, \boldsymbol{H}, J\}$ and $\left\{\boldsymbol{\Sigma}_{\boldsymbol{F}}, \boldsymbol{\Sigma}_{\boldsymbol{H}}, \Sigma_{J}\right\}$ is one to one and invertible. It is therefore possible to define a complementary energy function by means of a Legendre transform as:

$$
\Upsilon\left(\boldsymbol{\Sigma}_{\boldsymbol{F}}, \boldsymbol{\Sigma}_{\boldsymbol{H}}, \Sigma_{J}\right)=\max _{\boldsymbol{F}, \boldsymbol{H}, J}\left\{\boldsymbol{\Sigma}_{\boldsymbol{F}}: \boldsymbol{F}+\boldsymbol{\Sigma}_{\boldsymbol{H}}: \boldsymbol{H}+\Sigma_{J} J-W(\boldsymbol{F}, \boldsymbol{H}, J)\right\} .
$$

For instance, in the particular case of a Mooney-Rivlin material, the complementary energy is easily evaluated as:

$$
\Upsilon_{M R}\left(\boldsymbol{\Sigma}_{\boldsymbol{F}}, \boldsymbol{\Sigma}_{\boldsymbol{H}}, \Sigma_{J}\right)=\frac{1}{4 \alpha} \boldsymbol{\Sigma}_{\boldsymbol{F}}: \boldsymbol{\Sigma}_{\boldsymbol{F}}+\frac{1}{4 \beta} \boldsymbol{\Sigma}_{\boldsymbol{H}}: \boldsymbol{\Sigma}_{\boldsymbol{H}}+g\left(\Sigma_{J}\right)
$$

where the complementary function $g$ is defined by the Legendre transform expression:

$$
g\left(\Sigma_{J}\right)=\Sigma_{J} J\left(\Sigma_{J}\right)-f\left(J\left(\Sigma_{J}\right)\right)
$$

and the relationship $J\left(\Sigma_{J}\right)$ is obtained inverting equation (35c), that is, $J\left(f^{\prime}(x)\right)=x$. Note that if either $\alpha$ or $\beta$ is zero, the corresponding term in the complementary energy also vanishes. For instance, the case $\beta=0$ corresponds to a compressible neo-Hookean material, for which:

$$
\Upsilon_{N H}\left(\boldsymbol{\Sigma}_{\boldsymbol{F}}, J\right)=\frac{1}{4 \alpha} \boldsymbol{\Sigma}_{\boldsymbol{F}}: \boldsymbol{\Sigma}_{\boldsymbol{F}}+g\left(\Sigma_{J}\right) ; \quad \boldsymbol{\Sigma}_{\boldsymbol{H}}=\mathbf{0} .
$$

\subsection{Tangent elasticity operator}

A tangent elasticity operator will be required in order to ensure quadratic convergence of a Newton-Raphson type of solution process. This is typically evaluated in terms of a fourth order tangent elasticity tensor defined by:

$$
D^{2} \Psi[\delta \boldsymbol{v} ; \boldsymbol{u}]=\nabla_{0} \delta \boldsymbol{v}: D \boldsymbol{P}[u]=\nabla_{0} \delta \boldsymbol{v}: \mathcal{C}: \nabla_{0} \boldsymbol{u} ; \quad \mathcal{C}=\frac{\partial \boldsymbol{P}}{\partial \boldsymbol{F}}=\frac{\partial^{2} \Psi}{\partial \boldsymbol{F} \partial \boldsymbol{F}}
$$

Using equation (39) for the first Piola-Kirchhoff tensor and following a chain rule derivation similar to equation (38) and making use of equations (23) and (24) for the derivatives of $\boldsymbol{H}$, yields after simple algebra:

$$
\begin{aligned}
D^{2} \Psi[\delta \boldsymbol{v} ; \boldsymbol{u}] & =\boldsymbol{\nabla}_{0} \delta \boldsymbol{v}: D \boldsymbol{P}[u] \\
& =\boldsymbol{\nabla}_{0} \delta \boldsymbol{v}: D \boldsymbol{\Sigma}_{\boldsymbol{F}}[u]+\left(\boldsymbol{\nabla}_{0} \delta \boldsymbol{v} \times \boldsymbol{F}\right): D \boldsymbol{\Sigma}_{\boldsymbol{H}}[u]+\left(\boldsymbol{\nabla}_{0} \delta \boldsymbol{v}: \boldsymbol{H}\right) D \Sigma_{J}[u] \\
& +\left(\boldsymbol{\Sigma}_{\boldsymbol{H}}+\Sigma_{J} \boldsymbol{F}\right):\left(\boldsymbol{\nabla}_{0} \delta \boldsymbol{v} \times \boldsymbol{\nabla}_{0} \boldsymbol{u}\right) .
\end{aligned}
$$


In general, the conjugate stresses $\left\{\boldsymbol{\Sigma}_{\boldsymbol{F}}, \boldsymbol{\Sigma}_{\boldsymbol{H}}, \Sigma_{J}\right\}$ will be functions of each of the strain variables $\{\boldsymbol{F}, \boldsymbol{H}, J\}$ and the resulting tangent operator can be represented as:

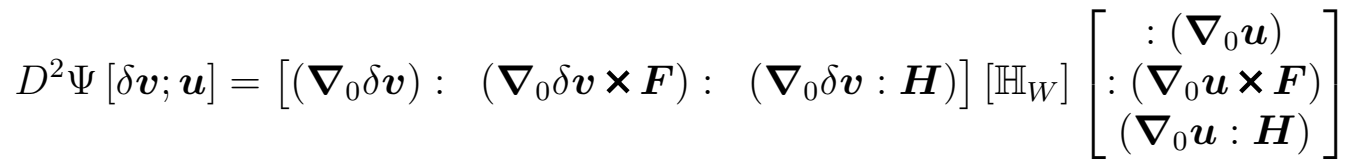

$$
\begin{aligned}
& +\left(\boldsymbol{\Sigma}_{\boldsymbol{H}}+\Sigma_{J} \boldsymbol{F}\right):\left(\boldsymbol{\nabla}_{0} \delta \boldsymbol{v} \times \boldsymbol{\nabla}_{0} \boldsymbol{u}\right)
\end{aligned}
$$

where the Hessian operator $\mathbb{H}_{W}$ denotes the symmetric positive definite operator containing the second derivatives of $W(\boldsymbol{F}, \boldsymbol{H}, J)$ :

$$
\left[\mathbb{H}_{W}\right]=\left[\begin{array}{ccc}
\frac{\partial^{2} W}{\partial \boldsymbol{F} \partial \boldsymbol{F}} & \frac{\partial^{2} W}{\partial \boldsymbol{F} \partial \boldsymbol{H}} & \frac{\partial^{2} W}{\partial \boldsymbol{F} \partial J} \\
\frac{\partial^{2} W}{\partial \boldsymbol{H} \partial \boldsymbol{F}} & \frac{\partial^{2} W}{\partial \boldsymbol{H} \partial \boldsymbol{H}} & \frac{\partial^{2} W}{\partial \boldsymbol{H} \partial J} \\
\frac{\partial^{2} W}{\partial J \partial \boldsymbol{F}} & \frac{\partial^{2} W}{\partial J \partial \boldsymbol{H}} & \frac{\partial^{2} W}{\partial J \partial J}
\end{array}\right] .
$$

Note that the first term in equation (48) is necessarily positive for $\delta \boldsymbol{v}=$ $\boldsymbol{u}$ and therefore buckling can only be induced by the "initial stress" term $\left(\boldsymbol{\Sigma}_{\boldsymbol{H}}+\Sigma_{J} \boldsymbol{F}\right):\left(\boldsymbol{\nabla}_{0} \delta \boldsymbol{v} \times \boldsymbol{\nabla}_{0} \boldsymbol{u}\right)$.

Remark 2: Equation (48) makes it easy to highlight the relationship between policonvexity and ellipticity. Ellipticity requires that the double contraction of the elasticity tensor by an arbitrary rank-one tensor $\boldsymbol{v} \otimes \boldsymbol{V}$ should be positive, that is,

$$
(\boldsymbol{v} \otimes \boldsymbol{V}): \mathcal{C}:(\boldsymbol{v} \otimes \boldsymbol{V})>0 .
$$

Taking $\boldsymbol{\nabla}_{0} \delta \boldsymbol{v}=\boldsymbol{\nabla}_{0} \boldsymbol{u}=\boldsymbol{v} \otimes \boldsymbol{V}$ in equation (48) makes the initial stress term vanish since,

$$
\nabla_{0} \delta \boldsymbol{v} \times \nabla_{0} \boldsymbol{u}=(\boldsymbol{v} \otimes \boldsymbol{V}) \times(\boldsymbol{v} \otimes \boldsymbol{V})=(\boldsymbol{v} \times \boldsymbol{v}) \otimes(\boldsymbol{V} \times \boldsymbol{V})=\mathbf{0} .
$$

This leaves only the contribution from the first positive definite term in equation (48). It is therefore easy to note that polyconvexity implies ellipticity [13]. 
It is helpful to consider the simple case of a compressible Mooney-Rivlin material for which the off-diagonal terms of the Hessian matrix vanish and the tangent elastic operator becomes:

$$
\begin{aligned}
D^{2} \Psi_{M R}[\delta \boldsymbol{v} ; \boldsymbol{u}] & =2 \alpha \boldsymbol{\nabla}_{0} \delta \boldsymbol{v}: \boldsymbol{\nabla}_{0} \boldsymbol{u}+2 \beta\left(\boldsymbol{\nabla}_{0} \delta \boldsymbol{v} \times \boldsymbol{F}\right):\left(\boldsymbol{\nabla}_{0} \boldsymbol{u} \times \boldsymbol{F}\right) \\
& +f^{\prime \prime}(J)\left(\boldsymbol{\nabla}_{0} \delta \boldsymbol{v}: \boldsymbol{H}\right)\left(\boldsymbol{\nabla}_{0} \boldsymbol{u}: \boldsymbol{H}\right)+\left(\boldsymbol{\Sigma}_{\boldsymbol{H}}+\Sigma_{J} \boldsymbol{F}\right):\left(\boldsymbol{\nabla}_{0} \delta \boldsymbol{v} \times \boldsymbol{\nabla}_{0} \boldsymbol{u}\right) .
\end{aligned}
$$

It is now possible to derive appropriate values for the material parameters $\alpha, \beta$ and the function $f(J)$ by ensuring that at the reference configuration the above operator coincides with the classic linear elasticity operator, which is typically expressed in terms of the Lame coefficients $\{\lambda, \mu\}$ as:

$D^{2} \Psi_{L I N}[\delta \boldsymbol{v} ; \boldsymbol{u}]=\lambda\left(\boldsymbol{\nabla}_{0} \delta \boldsymbol{v}: \boldsymbol{I}\right)\left(\boldsymbol{\nabla}_{0} \boldsymbol{u}: \boldsymbol{I}\right)+\mu\left(\boldsymbol{\nabla}_{0} \delta \boldsymbol{v}: \boldsymbol{\nabla}_{0} \boldsymbol{u}+\boldsymbol{\nabla}_{0} \delta \boldsymbol{v}^{T}: \boldsymbol{\nabla}_{0} \boldsymbol{u}\right)$

Substituting $\boldsymbol{F}=\boldsymbol{H}=\boldsymbol{I} ; J=1$ into equation (52), making repeated use of property (12) for the tensor cross product and taking into account the zero initial stress condition (41) gives after lengthy but simple algebra:

$$
\begin{aligned}
\left.D^{2} \Psi_{M R}[\delta \boldsymbol{v} ; \boldsymbol{u}]\right|_{\boldsymbol{I}} & =(2 \alpha+2 \beta)\left(\boldsymbol{\nabla}_{0} \delta \boldsymbol{v}: \boldsymbol{\nabla}_{0} \boldsymbol{u}+\nabla_{0} \delta \boldsymbol{v}^{T}: \boldsymbol{\nabla}_{0} \boldsymbol{u}\right) \\
& +\left(f^{\prime \prime}(1)-2 \alpha\right)\left(\boldsymbol{\nabla}_{0} \delta \boldsymbol{v}: \boldsymbol{I}\right)\left(\boldsymbol{\nabla}_{0} \boldsymbol{u}: \boldsymbol{I}\right)
\end{aligned}
$$

Identifying coefficients leads to the simple condition relating $\alpha, \beta$ to the shear modulus $\mu$ :

$$
\alpha+\beta=\frac{\mu}{2}
$$

and the conditions for the first two derivatives of $f$ at the origin:

$$
\begin{aligned}
& f^{\prime}(1)=-2 \alpha-4 \beta \\
& f^{\prime \prime}(1)=\lambda+2 \alpha .
\end{aligned}
$$

A commonly used convex expression for $f$ that satisfies these requirements is:

$$
f(J)=-4 \beta J-2 \alpha \ln J+\frac{\lambda}{2 \varepsilon^{2}}\left(J^{\varepsilon}+J^{-\varepsilon}\right) ; \quad \varepsilon \geq 1 .
$$

\subsection{Nearly incompressible Mooney-Rivlin material}

Very often it is convenient or even necessary to separate the distortional component from the volumetric response of the material. This is invariably the case when attempting to model either nearly-incompressible materials 
or truly incompressible solids using approximate finite element spaces. Typically, this is achieved by separating the strain energy into isochoric and volumetric components, $\hat{\Psi}$ and $U$ respectively, as:

$$
\Psi\left(\boldsymbol{\nabla}_{0} \boldsymbol{x}\right)=\hat{\Psi}\left(\boldsymbol{\nabla}_{0} \boldsymbol{x}\right)+U\left(\operatorname{det} \boldsymbol{\nabla}_{0} \boldsymbol{x}\right) ; \quad \hat{\Psi}\left(\boldsymbol{\nabla}_{0} \boldsymbol{x}\right)=\Psi\left(\left(\operatorname{det} \boldsymbol{\nabla}_{0} \boldsymbol{x}\right)^{-1 / 3} \boldsymbol{\nabla}_{0} \boldsymbol{x}\right) .
$$

The first term in this energy expression leads to the deviatoric component of the Piola-Kirchhoff tensor and the derivative of the function $U$ accounts for the pressure $p$. In the context of polyconvex elasticity, it is also possible to construct a similar decomposition in the form:

$$
W(\boldsymbol{F}, \boldsymbol{H}, J)=\hat{W}(\boldsymbol{F}, \boldsymbol{H}, J)+U(J) .
$$

For the purpose of deriving the conditions that needs to satisfy in order to ensure that alone accounts for the pressure, recall first that the pressure is obtained from the first Piola-kirchhoff tensor via the contraction:

$$
p=\frac{1}{3} J^{-1} \boldsymbol{P}: \boldsymbol{F} \text {. }
$$

Note that the sign convention used above is positive pressure in tension, negative in compression. Substituting the relationship between the PiolaKirchhoff stress tensor and the conjugate stresses given by equation (39), yields a relationship between the pressure and the conjugate stresses as:

$$
\begin{aligned}
p & =\frac{1}{3} J^{-1} \boldsymbol{P}: \boldsymbol{F} \\
& =\frac{1}{3} J^{-1}\left(\boldsymbol{\Sigma}_{\boldsymbol{F}}+\boldsymbol{\Sigma}_{\boldsymbol{H}} \times \boldsymbol{F}+\Sigma_{J} \boldsymbol{H}\right): \boldsymbol{F} \\
& =\frac{1}{3} J^{-1}\left(\boldsymbol{\Sigma}_{\boldsymbol{F}}: \boldsymbol{F}+2 \boldsymbol{\Sigma}_{\boldsymbol{H}}: \boldsymbol{H}+3 \Sigma_{J} J\right) .
\end{aligned}
$$

Substituting the constitutive relationships (34) and decomposition (59) into this equation for the pressure gives:

$$
p=\frac{1}{3} J^{-1}\left(\frac{\partial \hat{W}}{\partial \boldsymbol{F}}: \boldsymbol{F}+2 \frac{\partial \hat{W}}{\partial \boldsymbol{H}}: \boldsymbol{H}+3 J \frac{\partial \hat{W}}{\partial J}\right)+U^{\prime}(J) .
$$

Therefore the condition that $\hat{W}$ needs to satisfy in order to ensure a correct decomposition into volumetric and deviatoric components is:

$$
\frac{\partial \hat{W}}{\partial \boldsymbol{F}}: \boldsymbol{F}+2 \frac{\partial \hat{W}}{\partial \boldsymbol{H}}: \boldsymbol{H}+3 J \frac{\partial \hat{W}}{\partial J}=0 .
$$


In order to satisfy this requirement, it is sufficient for $\hat{W}$ to satisfy the following mixed homogeneous condition:

$$
\hat{W}\left(\alpha \boldsymbol{F}, \alpha^{2} \boldsymbol{H}, \alpha^{3} J\right)=\hat{W}(\boldsymbol{F}, \boldsymbol{H}, J) .
$$

Differentiating this equation with respect to $\alpha$ at $\alpha=1$ quickly leads to condition (62). A simple way to ensure that this requirement is satisfied would be to construct $\hat{W}$ in terms of the isochoric components of $\boldsymbol{F}$ and $\boldsymbol{H}$ as:

$$
\hat{W}(\boldsymbol{F}, \boldsymbol{H})=W(\hat{\boldsymbol{F}}, \hat{\boldsymbol{H}}, 1)
$$

where the isochoric components could be defined in the usual fashion [1]:

$$
\hat{\boldsymbol{F}}=(\operatorname{det} \boldsymbol{F})^{-1 / 3} \boldsymbol{F} ; \quad \hat{\boldsymbol{H}}=(\operatorname{det} \boldsymbol{H})^{-1 / 3} \boldsymbol{H} .
$$

Unfortunately, the resulting strain energy function constructed in this manner will not be convex with respect to $\boldsymbol{F}$ and $\boldsymbol{H}$. Alternative expressions can be derived by re-defining the iscochoric components of $\boldsymbol{F}$ and $\boldsymbol{H}$ as:

$$
\hat{\boldsymbol{F}}=J^{-1 / 3} \boldsymbol{F} ; \quad \hat{\boldsymbol{H}}=J^{-2 / 3} \boldsymbol{H} .
$$

Or, alternatively, noting that $\boldsymbol{F}: \boldsymbol{H}=3 J$, as

$$
\hat{\boldsymbol{F}}=\left(\frac{1}{3} \boldsymbol{F}: \boldsymbol{H}\right)^{-1 / 3} \boldsymbol{F} ; \quad \hat{\boldsymbol{H}}=\left(\frac{1}{3} \boldsymbol{F}: \boldsymbol{H}\right)^{-2 / 3} \boldsymbol{H}
$$

For instance, in the case of the Mooney-Rivlin material an equivalent polyconvex isochoric energy function is obtained as [24]:

$$
\hat{W}(\boldsymbol{F}, \boldsymbol{H}, J)=\eta J^{-2 / 3}(\boldsymbol{F}: \boldsymbol{F})+\gamma J^{-2}(\boldsymbol{H}: \boldsymbol{H})^{3 / 2} .
$$

The most commonly used expression for the volumetric strain energy component $U(J)$ is given by:

$$
U(J)=\frac{1}{2} \kappa(J-1)^{2} .
$$

Note that the dependency of the isochoric strain energy function $\hat{W}$ with respect to $J$ implies that the pressure $p$ and the conjugate stress $\Sigma_{J}$ are not identical. They are in fact related by:

$$
\Sigma_{J}=\hat{\Sigma}_{J}+p ; \quad \hat{\Sigma}_{J}=\frac{\partial \hat{W}}{\partial J} ; \quad p=U^{\prime}(J) .
$$


Finally, the tangent elastic operator of this nearly incompressible model can be derived in a manner similar to equation (48) to give:

$$
\begin{aligned}
& D^{2} \Psi[\delta \boldsymbol{v} ; \boldsymbol{u}]=D^{2} \hat{\Psi}[\delta \boldsymbol{v} ; \boldsymbol{u}]+U^{\prime \prime}\left(\nabla_{0} \delta \boldsymbol{v}: \boldsymbol{H}\right)\left(\nabla_{0} \boldsymbol{u}: \boldsymbol{H}\right)
\end{aligned}
$$

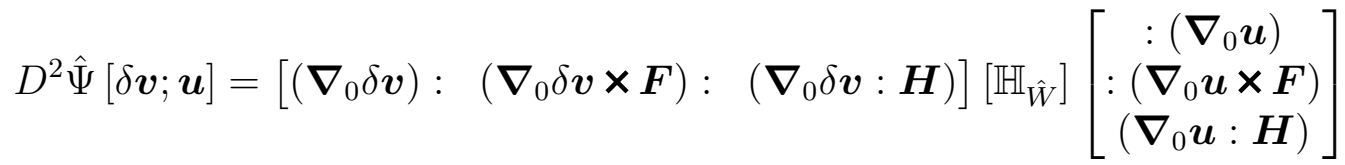

$$
\begin{aligned}
& +\left(\boldsymbol{\Sigma}_{\boldsymbol{H}}+\Sigma_{J} \boldsymbol{F}\right):\left(\boldsymbol{\nabla}_{0} \delta \boldsymbol{v} \times \boldsymbol{\nabla}_{0} \boldsymbol{u}\right)
\end{aligned}
$$

\subsection{The Second Piola-Kirchhoff, Kirchhoff and Cauchy stress tensors}

The formulation developed so far has been expressed in terms of the main kinematic variables $\boldsymbol{F}, \boldsymbol{H}$ and $J$. However, material frame indifference implies that the dependency of the strain energy with respect to $\boldsymbol{F}, \boldsymbol{H}$ must be via the right Cauchy-Green tensor $\boldsymbol{C}=\boldsymbol{F}^{T} \boldsymbol{F}$ and its cofactor $\boldsymbol{G}=$

$\boldsymbol{H}^{T} \boldsymbol{H}=\frac{1}{2} \boldsymbol{C} \times \boldsymbol{C}$. It is therefore possible to express the strain energy as a function of these symmetric tensors as:

$$
\Psi\left(\boldsymbol{\nabla}_{0} \boldsymbol{x}\right)=\tilde{W}(\boldsymbol{C}, \boldsymbol{G}, C)
$$

where, for consistency, $C=\operatorname{det} \boldsymbol{C}=J^{2}$ is being used instead of $J$ as the variable describing the volumetric change. Note, however, that the function $\tilde{W}$ need not to be strictly convex with respect to its variables. For instance, in the case of a Mooney-Rivlin material, $\tilde{W}$ is linear with respect to both $\boldsymbol{C}$ and $\boldsymbol{G}$ as,

$$
\tilde{W}_{M R}(\boldsymbol{C}, \boldsymbol{G}, C)=\alpha \boldsymbol{C}: \boldsymbol{I}+\beta \boldsymbol{G}: \boldsymbol{I}+\tilde{f}(C) ; \quad \tilde{f}(C)=f(\sqrt{C}) .
$$

Using the work conjugacy expresion between the second Piola-Kirchhoff $\boldsymbol{S}$ and the right Cauchy-Green tensor $\boldsymbol{C}$ given by:

$$
D \Psi[\delta \boldsymbol{v}]=\boldsymbol{S}: \frac{1}{2} D \boldsymbol{C}[\delta \boldsymbol{v}] ; \quad \boldsymbol{S}=\left.2 \frac{\partial \Psi(\boldsymbol{C})}{\partial \boldsymbol{C}}\right|_{\boldsymbol{C}=\left(\boldsymbol{\nabla}_{0} \boldsymbol{x}\right)^{T}\left(\boldsymbol{\nabla}_{0} \boldsymbol{x}\right)}
$$

and defining the conjugate stresses to $\boldsymbol{C}, \boldsymbol{G}$ and $C$ as:

$$
\boldsymbol{\Sigma}_{\boldsymbol{C}}=2 \frac{\partial \tilde{W}}{\partial \boldsymbol{C}} ; \quad \boldsymbol{\Sigma}_{\boldsymbol{G}}=2 \frac{\partial \tilde{W}}{\partial \boldsymbol{G}} ; \quad \Sigma_{C}=2 \frac{\partial \tilde{W}}{\partial C}
$$


enables an expression for the second Piola-Kirchhoff tensor to be derived using the same steps employed in equation (38) for the derivation of the first Piola-Kirchhoff tensor to give:

$$
S=\Sigma_{C}+\Sigma_{G} \times C+\Sigma_{C} G .
$$

It is also possible to derive the total Lagrangian elasticity tensor in terms of the Hessian matrix of $\tilde{W}$ following similar steps to those employed in previous sections. This derivation will not be pursued here as it will not be used in the computational framework proposed later.

In addition to the first and second Piola-Kirchhoff stresses, it is necessary to derive expressions for the Cauchy and Kirchhoff stresses as often these tensors are needed in order to express plasticity models or simply to display solution results. Such expressions can be relatively easily derived from the standard relationship between these tensors [1]:

$$
J \boldsymbol{\sigma}=\boldsymbol{\tau}=\boldsymbol{P} \boldsymbol{F}^{T}
$$

Substituting equation (39) for the first Piola-Kirchhoff tensor and recalling that $\boldsymbol{H} \boldsymbol{F}^{T}=J \boldsymbol{I}$ gives,

$$
J \boldsymbol{\sigma}=\boldsymbol{\tau}=\boldsymbol{\Sigma}_{\boldsymbol{F}} \boldsymbol{F}^{T}+\left(\boldsymbol{\Sigma}_{\boldsymbol{H}} \times \boldsymbol{F}\right) \boldsymbol{F}^{T}+J \Sigma_{J} \boldsymbol{I}
$$

The middle term in this expression can be transformed with the help of property (21) of the tensor cros product by taking $\boldsymbol{B}=\boldsymbol{F}, \boldsymbol{A}_{1}=J^{-1} \boldsymbol{\Sigma}_{\boldsymbol{H}} \boldsymbol{H}^{T}$ and $\boldsymbol{A}_{2}=\boldsymbol{I}$ to give,

$$
\left(\boldsymbol{\Sigma}_{\boldsymbol{H}} \times \boldsymbol{F}\right) \boldsymbol{F}^{T}=\boldsymbol{\Sigma}_{\boldsymbol{H}} \boldsymbol{H}^{T} \times \boldsymbol{I}
$$

Thus giving an expression for the Kirchhoff stresses as:

$$
J \boldsymbol{\sigma}=\boldsymbol{\tau}=\boldsymbol{\Sigma}_{\boldsymbol{F}} \boldsymbol{F}^{T}+\left(\boldsymbol{\Sigma}_{\boldsymbol{H}} \boldsymbol{H}^{T} \times \boldsymbol{I}\right)+J \Sigma_{J} \boldsymbol{I} .
$$

Alternatively, introducing the notation:

$$
\boldsymbol{\tau}_{\boldsymbol{F}}=\boldsymbol{\Sigma}_{\boldsymbol{F}} \boldsymbol{F}^{T} ; \quad \boldsymbol{\tau}_{\boldsymbol{H}}=\boldsymbol{\Sigma}_{\boldsymbol{H}} \boldsymbol{H}^{T} ; \quad \tau_{J}=J \Sigma_{J}
$$

gives,

$$
J \boldsymbol{\sigma}=\boldsymbol{\tau}=\boldsymbol{\tau}_{\boldsymbol{F}}+\tau_{\boldsymbol{H}} \times \boldsymbol{I}+\tau_{J} \boldsymbol{I}
$$




\section{Variational formulations}

This section presents several possible variational formulations. The section starts reviewing the standard displacement based variational principle. For this case the present framework does not provide any practical advantages, other than the evaluation of the first Piola-Kirchhoff stress and the tangent modulus via equations (39) and (48), respectively. It is simply presented here in order to provide a useful background for comparison with mixed and complementary energy variational principles presented later in the section. The aim of the section is to present the concepts in as simple a manner as possible, rather than to provide precise mathematical statements given that, in practice, the concepts presented here will be implemented in well defined discrete finite element spaces described in the following section.

\subsection{Standard displacement based variational principle}

The solution of large strain elastic problems is often expressed by means of the total energy minimisation variational principle as:

$$
\Pi\left(\boldsymbol{x}^{*}\right)=\min _{\boldsymbol{x}}\left\{\int_{V} \Psi\left(\boldsymbol{\nabla}_{0} \boldsymbol{x}\right) d V-\int_{V} \boldsymbol{f}_{0} \cdot \boldsymbol{x} d V-\int_{\partial_{t} V} \boldsymbol{t}_{0} \cdot \boldsymbol{x} d A\right\}
$$

where $\boldsymbol{x}^{*}$ denotes the exact solution. The stationary condition of this functional leads to the principle of virtual work (or power), commonly written as:

$$
D \Pi[\delta \boldsymbol{v}]=\int_{V} \boldsymbol{P}_{\boldsymbol{x}}: \nabla_{0} \delta \boldsymbol{v} d V-\int_{V} \boldsymbol{f}_{0} \cdot \delta \boldsymbol{v} d V-\int_{\partial_{t} V} \boldsymbol{t}_{0} \cdot \delta \boldsymbol{v} d A=0 .
$$

In this expression, the first Piola-Kirchhoff tensor $\boldsymbol{P}_{\boldsymbol{x}}$ is evaluated in the standard fashion using equation (39) in terms of the gradient of the deformation $\boldsymbol{\nabla}_{0} \boldsymbol{x}$. For convenience and in order to simplify the notation, it is useful to introduce the definition of the geometrically compatible strain measures as:

$$
\boldsymbol{F}_{\boldsymbol{x}} \equiv \boldsymbol{\nabla}_{0} \boldsymbol{x} ; \quad \boldsymbol{H}_{\boldsymbol{x}} \equiv \frac{1}{2} \boldsymbol{\nabla}_{0} \boldsymbol{x} \times \boldsymbol{\nabla}_{0} \boldsymbol{x} ; \quad J_{\boldsymbol{x}} \equiv \operatorname{det} \boldsymbol{\nabla}_{0} \boldsymbol{x} .
$$

In this manner, the first Piola-Kirchhoff tensor $\boldsymbol{P}_{\boldsymbol{x}}$ becomes:

$$
\boldsymbol{P}_{x}=\Sigma_{F}^{x}+\Sigma_{H}^{x} \times \boldsymbol{F}_{x}+\Sigma_{J}^{x} H_{x}
$$


where the superscript $\boldsymbol{x}$ in the above stresses indicates that they are evaluated in terms of the geometric deformation gradient as:

$$
\begin{aligned}
\Sigma_{\boldsymbol{F}}^{\boldsymbol{x}} & =\boldsymbol{\Sigma}_{\boldsymbol{F}}\left(\boldsymbol{F}_{\boldsymbol{x}}, \boldsymbol{H}_{\boldsymbol{x}}, J_{\boldsymbol{x}}\right) \\
\boldsymbol{\Sigma}_{\boldsymbol{H}}^{\boldsymbol{x}} & =\boldsymbol{\Sigma}_{\boldsymbol{H}}\left(\boldsymbol{F}_{\boldsymbol{x}}, \boldsymbol{H}_{\boldsymbol{x}}, J_{\boldsymbol{x}}\right) \\
\Sigma_{J}^{\boldsymbol{x}} & =\Sigma_{J}\left(\boldsymbol{F}_{\boldsymbol{x}}, \boldsymbol{H}_{\boldsymbol{x}}, J_{\boldsymbol{x}}\right) .
\end{aligned}
$$

An iterative Newton-Raphson process to converge towards the solution is usually established by solving a linearized system for the increment $\boldsymbol{u}$ as:

$$
D^{2} \Pi[\delta \boldsymbol{v} ; \boldsymbol{u}]=-D \Pi\left(\boldsymbol{x}_{k}\right)[\delta \boldsymbol{v}] ; \quad \boldsymbol{x}_{k+1}=\boldsymbol{x}_{k}+\boldsymbol{u}
$$

where, in the absence of follower forces, the second derivative of the total energy functional is given by:

$$
D^{2} \Pi[\delta \boldsymbol{v} ; \boldsymbol{u}]=\int_{V} D^{2} \Psi\left[\boldsymbol{\nabla}_{0} \delta \boldsymbol{v}, \boldsymbol{\nabla}_{0} \boldsymbol{u}\right] d V
$$

The tangent operator is evaluated using equation (48), taking $\boldsymbol{F}=\boldsymbol{\nabla}_{0} \boldsymbol{x}$, $\boldsymbol{H}=\frac{1}{2} \boldsymbol{\nabla}_{0} \boldsymbol{x} \times \boldsymbol{\nabla}_{0} \boldsymbol{x}$ and $J=\operatorname{det} \boldsymbol{\nabla}_{0} \boldsymbol{x}$.

\subsection{Mixed Variational Principle}

An equivalent but alternative expression for the total energy variational principle can be written in terms of the geometry and strain variables as a constrained minimisation problem in the form:

$$
\Pi\left(\boldsymbol{x}^{*}\right)=\min _{\substack{\boldsymbol{x}, \boldsymbol{F}, \boldsymbol{H}, J, \text { s.t. } \\ \boldsymbol{F}=\boldsymbol{F}, \\ \text {, } \boldsymbol{H}_{x}, J=J_{x}}}\left\{\int_{V} W(\boldsymbol{F}, \boldsymbol{H}, J) d V-\int_{V} \boldsymbol{f}_{0} \cdot \boldsymbol{x} d V-\int_{\partial_{t} V} \boldsymbol{t}_{0} \cdot \boldsymbol{x} d A\right\} .
$$

Using a standard Lagrange multiplier approach to enforce the compati- 
bility constraints gives the following augmented mixed variational principle:

$$
\begin{aligned}
& \Pi_{M}\left(\boldsymbol{x}^{*}, \boldsymbol{F}^{*}, \boldsymbol{H}^{*}, J^{*}, \boldsymbol{\Sigma}_{\boldsymbol{F}}^{*}, \boldsymbol{\Sigma}_{\boldsymbol{H}}^{*}, \Sigma_{J}^{*}\right)=\min _{\boldsymbol{x}, \boldsymbol{F}, \boldsymbol{H}, J}\left\{\operatorname { m a x } _ { \boldsymbol { \Sigma } _ { \boldsymbol { F } , \boldsymbol { \Sigma } _ { \boldsymbol { H } } , \Sigma _ { J } } } \left\{\int_{V} W(\boldsymbol{F}, \boldsymbol{H}, J) d V\right.\right. \\
& +\int_{V} \boldsymbol{\Sigma}_{\boldsymbol{F}}:\left(\boldsymbol{F}_{\boldsymbol{x}}-\boldsymbol{F}\right) d V+\int_{V} \boldsymbol{\Sigma}_{\boldsymbol{H}}:\left(\boldsymbol{H}_{\boldsymbol{x}}-\boldsymbol{H}\right) d V+\int_{J}\left(J_{\boldsymbol{x}}-J\right) d V \\
& \left.\left.-\int_{V} \boldsymbol{f}_{0} \cdot \boldsymbol{x} d V-\int_{\partial_{t} V} \boldsymbol{t}_{0} \cdot \boldsymbol{x} d A\right\}\right\} .
\end{aligned}
$$

This expression belongs to the general class of $\mathrm{Hu}$-Washizu type of mixed variational principles which have been widely used for the development of enhanced finite element formulations [1]. Note that the stress variables $\left\{\boldsymbol{\Sigma}_{\boldsymbol{F}}, \boldsymbol{\Sigma}_{\boldsymbol{H}}, \Sigma_{J}\right\}$ in this expression, at this stage, are simply Lagrange multipliers and are as yet unconnected to the strain variables.

The stationary condition of the above augmented Lagrangian with respect to the first variable enforces equilibrium in the form of the principle of virtual work as:

$$
D_{1} \Pi_{M}[\delta \boldsymbol{v}]=\int_{V} \boldsymbol{P}_{M}: \nabla_{0} \delta \boldsymbol{v} d V-\int_{V} \boldsymbol{f}_{0} \cdot \delta \boldsymbol{v} d V-\int_{\partial_{t} V} \boldsymbol{t}_{0} \cdot \delta \boldsymbol{v} d A=0
$$

where the first Piola-Kirchhoff stress now emerges as:

$$
\boldsymbol{P}_{M}=\boldsymbol{\Sigma}_{\boldsymbol{F}}+\boldsymbol{\Sigma}_{\boldsymbol{H}} \times \boldsymbol{F}_{\boldsymbol{x}}+\Sigma_{J} \boldsymbol{H}_{\boldsymbol{x}}
$$

The stationary conditions with respect to the three strain variables enforce the constitute relationships between the stresses and the derivatives of the strain energy in a weak form:

$$
\begin{aligned}
D_{2,3,4} \Pi_{M}[\delta \boldsymbol{F}, \delta \boldsymbol{H}, \delta J] & =\int_{V}\left(\frac{\partial W}{\partial \boldsymbol{F}}-\boldsymbol{\Sigma}_{\boldsymbol{F}}\right): \delta \boldsymbol{F} d V+\int_{V}\left(\frac{\partial W}{\partial \boldsymbol{H}}-\boldsymbol{\Sigma}_{\boldsymbol{H}}\right): \delta \boldsymbol{H} d V \\
& +\int_{V}\left(\frac{\partial W}{\partial J}-\Sigma_{J}\right) \delta J d V=0 .
\end{aligned}
$$


Finally, the stationary conditions with respect to the stress variables enforce the geometric compatibility conditions between strains and geometry:

$$
\begin{aligned}
D_{5,6,7} \Pi_{M}\left[\delta \boldsymbol{\Sigma}_{\boldsymbol{F}}, \delta \boldsymbol{\Sigma}_{\boldsymbol{H}}, \delta \Sigma_{J}\right] & =\int_{V} \delta \boldsymbol{\Sigma}_{\boldsymbol{F}}:\left(\boldsymbol{F}_{\boldsymbol{x}}-\boldsymbol{F}\right) d V+\int_{V} \delta \boldsymbol{H}:\left(\boldsymbol{H}_{\boldsymbol{x}}-\boldsymbol{H}\right) d V \\
& +\int_{V} \delta J\left(J_{\boldsymbol{x}}-J\right) d V .
\end{aligned}
$$

Remark 3. It is important to note that for, certain choices of interpolation spaces insufficiently rich, the above variational formulation may not ensure that the kinematic compatibility constraints are enforced at every point in the domain. In fact, in cases such as incompressible elasticity, the interpolation spaces will be chosen for this very reason. However, this weak enforcement of compatibility can have unwanted consequences such as the lack of symmetry of Cauchy (or Kirchhoff) stresses if these are calculated by the product:

$$
\begin{aligned}
\boldsymbol{\tau}_{M} & =\boldsymbol{P}_{M} \boldsymbol{F}_{x}^{T} \\
& =\boldsymbol{\Sigma}_{\boldsymbol{F}} \boldsymbol{F}_{x}^{T}+\left(\boldsymbol{\Sigma}_{\boldsymbol{H}} \times \boldsymbol{F}_{x}\right) \boldsymbol{F}_{x}^{T}+\Sigma_{J} \boldsymbol{H}_{x} \boldsymbol{F}_{x}^{T} \\
& =\boldsymbol{\Sigma}_{\boldsymbol{F}} \boldsymbol{F}_{x}^{T}+\left(\boldsymbol{\Sigma}_{\boldsymbol{H}} \boldsymbol{H}_{x}^{T}\right) \times \boldsymbol{F}_{x}^{T}+J_{x} \Sigma_{J} \boldsymbol{I} .
\end{aligned}
$$

In the above expression it is very unlikely that the terms $\boldsymbol{\Sigma}_{\boldsymbol{F}} \boldsymbol{F}_{x}^{T}$ and $\boldsymbol{\Sigma}_{\boldsymbol{H}} \boldsymbol{H}_{x}^{T}$ will be symmetric given the weak enforcement of the compatibility constraints. Note, however, that this Kirchhoff stress tensor is not part of the solution process. Nevertheless, it will often be required so that appropriate stress fields, such as Cauchy stresses, can be given as output of the computation. For this purpose, it is important that these stresses are evaluated using equation (82) directly, where $\boldsymbol{\tau}_{\boldsymbol{F}}$ and $\boldsymbol{\tau}_{\boldsymbol{H}}$ are evaluated independently using fully compatible kinematic-stress pairs. For instance, by taking $\boldsymbol{\tau}_{\boldsymbol{F}}=\boldsymbol{\Sigma}_{\boldsymbol{F}}(\boldsymbol{F}) \boldsymbol{F}^{T}$ and $\boldsymbol{\tau}_{\boldsymbol{H}}=\boldsymbol{\Sigma}_{\boldsymbol{H}}(\boldsymbol{H}) \boldsymbol{H}^{T}$.

In order to complete the formulation, it is necessary to develop a NewtonRaphson iterative process and the appropriate tangent operators. For this purpose, note first that a process equivalent to equation (88) for the extended set of variables is established by first solving a linear system for the 
increments of this set of variables $\left\{\boldsymbol{u}, \Delta \boldsymbol{F}, \Delta \boldsymbol{H}, \Delta J, \Delta \boldsymbol{\Sigma}_{\boldsymbol{F}}, \Delta \boldsymbol{\Sigma}_{\boldsymbol{H}}, \Delta \Sigma_{J}\right\}$ as:

$$
\begin{aligned}
& D_{1 \ldots 7 ; 1 \ldots 7}^{2} \Pi_{M}\left[\delta \boldsymbol{v}, \delta \boldsymbol{F}, \delta \boldsymbol{H}, \delta J, \delta \boldsymbol{\Sigma}_{\boldsymbol{F}}, \delta \boldsymbol{\Sigma}_{\boldsymbol{H}}, \delta \Sigma_{J} ; \boldsymbol{u}, \Delta \boldsymbol{F}, \Delta \boldsymbol{H}, \Delta J, \Delta \boldsymbol{\Sigma}_{\boldsymbol{F}}, \Delta \boldsymbol{\Sigma}_{\boldsymbol{H}}, \Delta \Sigma_{J}\right]= \\
& -D_{1 \ldots 7} \Pi_{M}\left[\delta \boldsymbol{v}, \delta \boldsymbol{F}, \delta \boldsymbol{H}, \delta J, \delta \boldsymbol{\Sigma}_{\boldsymbol{F}}, \delta \boldsymbol{\Sigma}_{\boldsymbol{H}}, \delta \Sigma_{J}\right] ; \quad \forall \delta \boldsymbol{v}, \delta \boldsymbol{F}, \delta \boldsymbol{H} \delta J, \delta \boldsymbol{\Sigma}_{\boldsymbol{G}}, \delta \boldsymbol{\Sigma}_{\boldsymbol{H}}, \delta \Sigma_{J} .
\end{aligned}
$$

This is followed by the incremental updates:

$$
\begin{aligned}
& \boldsymbol{x}_{k+1}=\boldsymbol{x}_{k}+\boldsymbol{u} ; \quad \boldsymbol{F}_{k+1}=\boldsymbol{F}_{k}+\Delta \boldsymbol{F} ; \quad \boldsymbol{H}_{k+1}=\boldsymbol{H}_{k}+\Delta \boldsymbol{H} ; \quad J_{k+1}=J_{k}+\Delta J ; \\
& \boldsymbol{\Sigma}_{\boldsymbol{F}}^{k+1}=\boldsymbol{\Sigma}_{\boldsymbol{F}}^{k}+\Delta \boldsymbol{\Sigma}_{\boldsymbol{F}} ; \quad \boldsymbol{\Sigma}_{\boldsymbol{H}}^{k+1}=\boldsymbol{\Sigma}_{\boldsymbol{H}}^{k}+\Delta \boldsymbol{\Sigma}_{\boldsymbol{H}} ; \quad \Sigma_{J}^{k+1}=\Sigma_{J}^{k}+\Delta \Sigma_{J} .
\end{aligned}
$$

The second derivatives that make up the linear operator in equation (97) can be derived with relative ease component by component. For instance, the second derivative with respect to the geometry, is obtained differentiating again the principle of virtual work, equation (92), with the help of equation (93) to give the "initial stress" component of the tangent operator as:

$$
D_{1 ; 1}^{2} \Pi_{M}[\delta \boldsymbol{v} ; \boldsymbol{u}]=\int_{V}\left(\boldsymbol{\Sigma}_{\boldsymbol{H}}+\Sigma_{J} \boldsymbol{F}_{\boldsymbol{x}}\right):\left(\boldsymbol{\nabla}_{0} \delta \boldsymbol{v} \times \boldsymbol{\nabla}_{0} \boldsymbol{u}\right) d V
$$

The terms involving second derivatives with respect to the strain variables emerge from the Hessian of the strain energy function $W(\boldsymbol{F}, \boldsymbol{H}, J)$ as:

$D_{2,3,4,2,3,4}^{2} \Pi_{M}[\delta \boldsymbol{F}, \delta \boldsymbol{H}, \delta J ; \Delta \boldsymbol{F}, \Delta \boldsymbol{H}, \Delta J]=\int_{V}[\delta \boldsymbol{F}: \quad \delta \boldsymbol{H}: \quad \delta J]\left[\mathbb{H}_{W}\right]\left[\begin{array}{c}: \Delta \boldsymbol{F} \\ : \Delta \boldsymbol{H} \\ \Delta J\end{array}\right] d V$

The second derivative with respect to stresses vanishes as the functional is linear with respect to the stress tensors. There are, however, a number of cross derivative terms that do not vanish. These are, the cross derivatives with respect to strains and stresses and their symmetric counterpart, which are easily derived from either equation (94) or (95) to give:

$$
\begin{aligned}
& D_{2,3,4 ; 5,6,7}^{2} \Pi_{M}\left[\delta \boldsymbol{F}, \delta \boldsymbol{H}, \delta J ; \Delta \boldsymbol{\Sigma}_{\boldsymbol{F}}, \Delta \boldsymbol{\Sigma}_{\boldsymbol{H}}, \Delta \Sigma_{J}\right]=-\int_{V}\left(\delta \boldsymbol{F}: \Delta \boldsymbol{\Sigma}_{\boldsymbol{F}}+\delta \boldsymbol{H}: \Delta \boldsymbol{\Sigma}_{\boldsymbol{H}}+\delta J \Delta \Sigma_{J}\right) d V \\
& D_{5,6,7 ; 2,3,4}^{2} \Pi_{M}\left[\delta \boldsymbol{\Sigma}_{\boldsymbol{F}}, \delta \boldsymbol{\Sigma}_{\boldsymbol{H}}, \delta \Sigma_{J} ; \Delta \boldsymbol{F}, \Delta \boldsymbol{H}, \Delta J\right]=-\int_{V}\left(\delta \boldsymbol{\Sigma}_{\boldsymbol{F}}: \Delta \boldsymbol{F}+\delta \boldsymbol{\Sigma}_{\boldsymbol{H}}: \Delta \boldsymbol{H}+\delta \Sigma_{J} \Delta J\right) d V .
\end{aligned}
$$


And the cross derivatives with respect to geometry and stresses, which emerge after some simple algebra from equations (92), (93) and (95) as:

$$
\begin{aligned}
D_{1 ; 5,6,7}^{2} \Pi_{M}\left[\delta \boldsymbol{v} ; \Delta \boldsymbol{\Sigma}_{\boldsymbol{F}}, \Delta \boldsymbol{\Sigma}_{\boldsymbol{H}}, \Delta \Sigma_{J}\right] & =\int_{V}\left[\boldsymbol{\nabla}_{0} \delta \boldsymbol{v}: \Delta \boldsymbol{\Sigma}_{\boldsymbol{F}}+\boldsymbol{F}_{\boldsymbol{x}}:\left(\boldsymbol{\nabla}_{0} \delta \boldsymbol{v} \times \Delta \boldsymbol{\Sigma}_{\boldsymbol{H}}\right)\right. \\
& \left.+\Delta \Sigma_{J} \boldsymbol{H}_{\boldsymbol{x}}: \boldsymbol{\nabla}_{0} \delta \boldsymbol{v}\right] d V \\
D_{5,6,7 ; 1}^{2} \Pi_{M}\left[\delta \boldsymbol{\Sigma}_{\boldsymbol{F}}, \delta \boldsymbol{\Sigma}_{\boldsymbol{H}}, \delta \Sigma_{J} ; \boldsymbol{u}\right] & =\int_{V}\left[\boldsymbol{\nabla}_{0} \boldsymbol{u}: \delta \boldsymbol{\Sigma}_{\boldsymbol{F}}+\boldsymbol{F}_{\boldsymbol{x}}:\left(\boldsymbol{\nabla}_{0} \boldsymbol{u} \times \delta \boldsymbol{\Sigma}_{\boldsymbol{H}}\right)\right. \\
& \left.+\delta \Sigma_{J} \boldsymbol{H}_{\boldsymbol{x}}: \boldsymbol{\nabla}_{0} \boldsymbol{u}\right] d V .
\end{aligned}
$$

The set of equations derived in this section enables the use of arbitrary discretisation spaces for each of the problem variables. This level of flexibility may be useful but it is costly given the large number of unknowns generated in the process. An alternative approach that significantly reduces the number of problem variables is presented in the next section.

\subsection{Complementary Energy Principle}

In order to derive a variational principle in terms of the complementary energy, recall first the mixed variational principle (91) with a different ordering of terms:

$$
\begin{aligned}
& \Pi_{M}\left(\boldsymbol{x}^{*}, \boldsymbol{F}^{*}, \boldsymbol{H}^{*}, J^{*}, \boldsymbol{\Sigma}_{\boldsymbol{F}}^{*}, \boldsymbol{\Sigma}_{\boldsymbol{H}}^{*}, \Sigma_{J}^{*}\right)=\min _{\boldsymbol{x}, \boldsymbol{F}, \boldsymbol{H}, J}\left\{\max _{\boldsymbol{\Sigma}_{\boldsymbol{F}}, \boldsymbol{\Sigma}_{\boldsymbol{H}}, \Sigma_{J}}\{\right. \\
& -\int_{V}\left[\boldsymbol{\Sigma}_{\boldsymbol{F}}: \boldsymbol{F}+\boldsymbol{\Sigma}_{\boldsymbol{H}}: \boldsymbol{H}+\Sigma_{J} J-W(\boldsymbol{F}, \boldsymbol{H}, J)\right] d V-\int_{V} \boldsymbol{f}_{0} \cdot \boldsymbol{x} d V-\int_{\partial_{t} V} \boldsymbol{t}_{0} \cdot \boldsymbol{x} d A \\
& \left.\left.+\int_{V} \boldsymbol{\Sigma}_{\boldsymbol{F}}: \boldsymbol{F}_{\boldsymbol{x}} d V+\int_{V} \boldsymbol{\Sigma}_{\boldsymbol{H}}: \boldsymbol{H}_{\boldsymbol{x}} d V+\int_{V} \Sigma_{J} J_{\boldsymbol{x}} d V\right\}\right\} .
\end{aligned}
$$

Comparing the term in square brackets in the first integral with the definition of the complementary energy given by equation (42), enables a com- 
plementary variational principle to be established $\mathrm{as}^{3}$ :

$$
\begin{aligned}
& \Pi_{M}\left(\boldsymbol{x}^{*}, \boldsymbol{\Sigma}_{\boldsymbol{F}}^{*}, \boldsymbol{\Sigma}_{\boldsymbol{H}}^{*}, \Sigma_{J}^{*}\right)=\min _{\boldsymbol{x} \in H}\left\{\operatorname { m a x } _ { \boldsymbol { \Sigma } _ { \boldsymbol { F } } , \boldsymbol { \Sigma } _ { \boldsymbol { H } } , \Sigma _ { J } } \left\{-\int_{V} \Upsilon\left(\boldsymbol{\Sigma}_{\boldsymbol{F}}, \boldsymbol{\Sigma}_{\boldsymbol{H}}, \Sigma_{J}\right) d V\right.\right. \\
& \left.\left.+\int_{V} \boldsymbol{\Sigma}_{\boldsymbol{F}}: \boldsymbol{F}_{\boldsymbol{x}} d V+\int_{V} \boldsymbol{\Sigma}_{\boldsymbol{H}}: \boldsymbol{H}_{\boldsymbol{x}} d V+\int_{V} \Sigma_{J} J_{\boldsymbol{x}} d V-\int_{V} \boldsymbol{f}_{0} \cdot \boldsymbol{x} d V-\int_{\partial_{t} V} \boldsymbol{t}_{0} \cdot \boldsymbol{x} d A\right\}\right\} .
\end{aligned}
$$

This represents a Helinger-Reissner type of variational principle [1]. The stationary condition of this principle with respect to its first variable, the geometry, enforces equilibrium in a manner identical to equations (92) and (93), that is,

$$
\begin{gathered}
D_{1} \Pi_{C}[\delta \boldsymbol{v}]=D_{1} \Pi_{M}[\delta \boldsymbol{v}]=\int_{V} \boldsymbol{P}_{M}: \boldsymbol{\nabla}_{0} \delta \boldsymbol{v} d V-\int_{V} \boldsymbol{f}_{0} \cdot \delta \boldsymbol{v} d V-\int_{\partial_{t} V} \boldsymbol{t}_{0} \cdot \delta \boldsymbol{v} d A=0 \\
\boldsymbol{P}_{C}=\boldsymbol{P}_{M}=\boldsymbol{\Sigma}_{\boldsymbol{F}}+\boldsymbol{\Sigma}_{\boldsymbol{H}} \mathbf{x} \boldsymbol{F}_{\boldsymbol{x}}+\Sigma_{J} \boldsymbol{H}_{\boldsymbol{x}}
\end{gathered}
$$

Similarly, the stationary conditions with respect to stresses, enforce the geometric compatibility conditions, now expressed as,

$$
\begin{aligned}
D_{2,3,4} \Pi_{C}\left[\delta \boldsymbol{\Sigma}_{\boldsymbol{F}}, \delta \boldsymbol{\Sigma}_{\boldsymbol{H}}, \delta \Sigma_{J}\right]= & \int_{V} \delta \boldsymbol{\Sigma}_{\boldsymbol{F}}:\left(\boldsymbol{F}_{\boldsymbol{x}}-\frac{\partial \Upsilon}{\partial \boldsymbol{\Sigma}_{\boldsymbol{F}}}\right) d V+\int_{V} \delta \boldsymbol{H}:\left(\boldsymbol{H}_{\boldsymbol{x}}-\frac{\partial \Upsilon}{\partial \boldsymbol{\Sigma}_{\boldsymbol{H}}}\right) d V \\
& \int_{V} \delta \Sigma_{J}\left(J_{\boldsymbol{x}}-\frac{\partial \Upsilon}{\partial \Sigma_{J}}\right) d V .
\end{aligned}
$$

The second derivatives of the complementary energy functional required for a Newton-Raphson process are mostly the same as those derived in the previous section for the mixed variational principle. In particular, the fol-

\footnotetext{
${ }^{3}$ Note that this step relies on the strong duality property of the mixed functional which allows the order of the min and max operations with respect to strains and stresses to be swapped. This is the case here given the convexity of the strain energy function.
} 
lowing terms are identical:

$$
\begin{aligned}
D_{1 ; 1}^{2} \Pi_{C}[\delta \boldsymbol{v} ; \boldsymbol{u}] & =D_{1 ; 1}^{2} \Pi_{M}[\delta \boldsymbol{v} ; \boldsymbol{u}] \\
D_{2,3,4 ; 1}^{2} \Pi_{C}\left[\delta \boldsymbol{\Sigma}_{\boldsymbol{F}}, \delta \boldsymbol{\Sigma}_{\boldsymbol{H}}, \delta \Sigma_{J} ; \boldsymbol{u}\right] & =D_{5,6,7 ; 1}^{2} \Pi_{M}\left[\delta \boldsymbol{\Sigma}_{\boldsymbol{F}}, \delta \boldsymbol{\Sigma}_{\boldsymbol{H}}, \delta \Sigma_{J} ; \boldsymbol{u}\right] \\
D_{1 ; 2,3,4}^{2} \Pi_{C}\left[\delta \boldsymbol{v} ; \Delta \boldsymbol{\Sigma}_{\boldsymbol{F}}, \Delta \boldsymbol{\Sigma}_{\boldsymbol{H}}, \Delta \Sigma_{J}\right] & =D_{1 ; 5,6,7}^{2} \Pi_{M}\left[\delta \boldsymbol{v} ; \Delta \boldsymbol{\Sigma}_{\boldsymbol{F}}, \Delta \boldsymbol{\Sigma}_{\boldsymbol{H}}, \Delta \Sigma_{J}\right] .
\end{aligned}
$$

A new term, however, emerges when taking the second derivatives with respect to stresses, leading to a constitutive expression similar to equation (100) but now involving the second derivative of the complementary energy function. This term can be evaluated by differentiating again equation (107) to give:

$$
\begin{array}{r}
D_{2,3,4 ; 2,3,4}^{2} \Pi_{C}\left[\delta \boldsymbol{\Sigma}_{\boldsymbol{F}}, \delta \boldsymbol{\Sigma}_{\boldsymbol{H}}, \delta \Sigma_{J} ; \Delta \boldsymbol{\Sigma}_{\boldsymbol{F}}, \Delta \boldsymbol{\Sigma}_{\boldsymbol{H}}, \Delta \Sigma_{J}\right]= \\
-\int_{V}\left[\delta \boldsymbol{\Sigma}_{\boldsymbol{F}}: \quad \delta \boldsymbol{\Sigma}_{\boldsymbol{H}}: \quad \delta \Sigma_{J}\right]\left[\mathbb{H}_{\Upsilon}\right]\left[\begin{array}{c}
: \Delta \boldsymbol{\Sigma}_{\boldsymbol{F}} \\
: \Delta \boldsymbol{\Sigma}_{\boldsymbol{H}} \\
\Delta \Sigma_{J}
\end{array}\right] d V .
\end{array}
$$

Where $\left[\mathbb{H}_{\Upsilon}\right]$ denotes the Hessian matrix of the complementary energy function. Note that this component is clearly negative on account of the variational principle involving a maximisation with respect to stresses. It is possible to change this sign by simply changing the overall the sign of the principle (105). This would also change the sign of $D_{1 ; 1}^{2} \Pi_{C}[\delta \boldsymbol{v} ; \boldsymbol{u}]$ but this term is neither positive nor negative since it contains tensor cross products of the gradient of $\delta \boldsymbol{v}$ and $\boldsymbol{u}$. For practical purposes, however, this will not be necessary, as the stress variables will typically be eliminated locally in each finite element. What is essential is that the Hessian matrix is invertible, which is ensured by the convexity of the function $\Upsilon$.

\subsection{Variational principles for incompressible and nearly incompressible mod- els}

Many applications of practical importance rely on the decomposition of the strain energy into isochoric and volumetric components. For such cases, it is possible to modify the variational formulations above in such a way that different approaches are used for the isochoric and volumetric components. In particular, it is often useful to follow a standard displacement based formulation for the isochoric component and a mixed approach for the volumetric terms [2]. In the present framework, this leads to the following hybrid mixed 
variational principle:

$$
\begin{aligned}
\hat{\Pi}_{M}\left(\boldsymbol{x}^{*}, J^{*}, p^{*}\right) & =\min _{\boldsymbol{x}, J}\left\{\operatorname { m a x } _ { p } \left\{\int_{V} \hat{W}\left(\boldsymbol{F}_{\boldsymbol{x}}, \boldsymbol{H}_{\boldsymbol{x}}, J_{\boldsymbol{x}}\right) d V+\int_{V} U(J) d V+\int_{V} p\left(J_{\boldsymbol{x}}-J\right) d V\right.\right. \\
& \left.\left.-\int_{V} \boldsymbol{f}_{0} \cdot \boldsymbol{x} d V-\int_{\partial_{t} V} \boldsymbol{t}_{0} \cdot \boldsymbol{x} d A\right\}\right\}
\end{aligned}
$$

where $\hat{W}$ and $U$ are the isochoric and volumetric components of the strain energy defined in Section 3.5. Note that, in general $\hat{W}$, will be a direct function of volume ratio. This volume ratio is expressed differently in the two terms making up the strain energy: it is directly evaluated from the geometry in the iscochoric strain energy, whereas it is expressed an independent variable $J$ in the volumetric component. The third integral term above enforces the compatibility between these two measures. The particular case of full incompressibility can be obtained by simply taking $J=1$ in the above expression to give:

$$
\begin{aligned}
\hat{\Pi}_{M}^{I}\left(\boldsymbol{x}^{*}, p^{*}\right) & =\min _{\boldsymbol{x}, J}\left\{\operatorname { m a x } _ { p } \left\{\int_{V} \hat{W}\left(\boldsymbol{F}_{\boldsymbol{x}}, \boldsymbol{H}_{\boldsymbol{x}}, J_{\boldsymbol{x}}\right) d V+\int_{V} p\left(J_{\boldsymbol{x}}-1\right) d V\right.\right. \\
& \left.\left.-\int_{V} \boldsymbol{f}_{0} \cdot \boldsymbol{x} d V-\int_{\partial_{t} V} \boldsymbol{t}_{0} \cdot \boldsymbol{x} d A\right\}\right\} .
\end{aligned}
$$

The stationary conditions of these hybrid functionals are evaluated in the same fashion as above. For instance, the first derivative with respect to geometry gives the principle of virtual work as:

$$
\begin{aligned}
D_{1} \hat{\Pi}_{M}[\delta \boldsymbol{v}]=D_{1} \hat{\Pi}_{M}^{I}[\delta \boldsymbol{v}] & =\int_{V} \boldsymbol{P}_{I}: \nabla_{0} \delta \boldsymbol{v} d V-\int_{V} \boldsymbol{f}_{0} \cdot \delta \boldsymbol{v} d V \\
& -\int_{\partial_{t} V} \boldsymbol{t}_{0} \cdot \delta \boldsymbol{v} d A=0
\end{aligned}
$$

where the first Piola-Kirchoff stress tensor is now evaluated as:

$$
\boldsymbol{P}_{I}=\boldsymbol{\Sigma}_{\boldsymbol{F}}^{\boldsymbol{x}}+\boldsymbol{\Sigma}_{\boldsymbol{H}}^{\boldsymbol{x}} \times \boldsymbol{F}_{\boldsymbol{x}}+\Sigma_{J} \boldsymbol{H}_{\boldsymbol{x}} ; \quad \Sigma_{J}=\hat{\Sigma}_{J}^{\boldsymbol{x}}+p
$$


and the last term in (113) indicates that the volumetric conjugate stress includes a component due to the independent variable $p$ as well as a contribution due to the isochoric strain energy function as:

$$
\hat{\Sigma_{J}^{\boldsymbol{x}}}=\frac{\partial \hat{W}\left(\boldsymbol{F}_{\boldsymbol{x}}, \boldsymbol{H}_{\boldsymbol{x}}, J_{\boldsymbol{x}}\right)}{\partial J_{\boldsymbol{x}}} .
$$

The first derivative with respect to $J$ enforces the volumetric component of the constitutive model as:

$$
D_{2} \hat{\Pi}_{M}[\delta J]=\int_{V}\left(U^{\prime}-p\right) \delta J d V=0 .
$$

Finally, the stationary condition with respect to the pressure enforces geometric compatibility between $J$ and $\operatorname{det} \boldsymbol{\nabla}_{0} \boldsymbol{x}$ as:

$$
\begin{aligned}
& D_{3} \hat{\Pi}_{M}[\delta p]=\int_{V}\left(J_{\boldsymbol{x}}-J\right) \delta p d V=0 \\
& D_{2} \hat{\Pi}_{M}^{I}[\delta p]=\int_{V}\left(J_{\boldsymbol{x}}-1\right) \delta p d V=0 .
\end{aligned}
$$

The evaluation of second derivatives required for a Newton-Raphson process proceeds along the same lines as in the previous sections. For instance, the second derivative with respect to geometry contains the isochoric tangent operator as given by equation (71):

$$
D_{1 ; 1}^{2} \hat{\Pi}_{M}[\delta \boldsymbol{v} ; \boldsymbol{u}]=D_{1 ; 1}^{2} \hat{\Pi}_{M}^{I}[\delta \boldsymbol{v} ; \boldsymbol{u}]=\int_{V} D^{2} \Psi[\delta \boldsymbol{v} ; \boldsymbol{u}] d V .
$$

The second derivative with respect to $J$ is easily evaluated from equation (115) to give:

$$
D_{2 ; 2}^{2} \hat{\Pi}_{M}[\delta J ; \Delta J]=\int_{V} U^{\prime \prime} \delta J \Delta J d V
$$

There are also cross derivative terms different from zero. For instance, the cross derivative with respect to $J$ and $p$ is:

$$
D_{2 ; 3}^{2} \hat{\Pi}_{M}[\delta J ; \Delta p]=-\int_{V} \Delta p \delta J d V ; \quad D_{3 ; 2}^{2} \hat{\Pi}_{M}[\delta p ; \Delta J]=-\int_{V} \delta p \Delta J d V .
$$


Finally, the cross derivative terms between pressure and geometry are:

$$
\begin{aligned}
& D_{1 ; 3}^{2} \hat{\Pi}_{M}[\delta \boldsymbol{v} ; \Delta p]=D_{1 ; 2}^{2} \hat{\Pi}_{M}^{I}[\delta \boldsymbol{v} ; \Delta p]=\int_{V}\left(\boldsymbol{H}_{x}: \nabla_{0} \delta \boldsymbol{v}\right) \Delta p d V \\
& D_{3 ; 1}^{2} \hat{\Pi}_{M}[\delta p ; \boldsymbol{u}]=D_{2 ; 1}^{2} \hat{\Pi}_{M}^{I}[\delta p ; \boldsymbol{u}]=\int_{V} \delta p\left(\boldsymbol{H}_{x}: \nabla_{0} \boldsymbol{u}\right) d V
\end{aligned}
$$

\section{Finite Element implementation}

It is not the purpose of this paper to provide an exhaustive analysis of different finite element interpolation strategies to discretise the equations derived in previous section. However, two particular examples will be provided in order to demonstrate the validity of the formulation presented.

\subsection{General remarks}

The implementation of the various variational principles described in the previous section is based on a finite element partition of the domain into a set of elements. Inside each element the problem variables are interpolated in terms of a set of shape functions $N_{a}$ as:

$$
\boldsymbol{x}=\sum_{a=1}^{n_{x}} \boldsymbol{x}_{a} N_{a}^{\boldsymbol{x}} ; \quad \boldsymbol{F}=\sum_{a=1}^{n_{F}} \boldsymbol{F}_{a} N_{a}^{\boldsymbol{F}} ; \quad \ldots ; \quad \boldsymbol{x}=\sum_{a=1}^{n_{\Sigma_{F}}} \boldsymbol{\Sigma}_{\boldsymbol{F} a} N_{a}^{\boldsymbol{\Sigma}_{\boldsymbol{F}}} ; \quad \ldots
$$

where $a$ denotes the nodes or other degrees of freedom used in the interpolation of the above variables. In general, different interpolations can (and are often) used to describe different variables. However, the same interpolation space will invariably be used for strain-stress conjugate pairs; that is, $N_{a}^{\boldsymbol{F}}=N_{a}^{\boldsymbol{\Sigma}_{\boldsymbol{F}}}$, etc. The virtual and incremental equivalents of the variables are also interpolated using the same spaces as:

$$
\begin{gathered}
\delta \boldsymbol{v}=\sum_{a=1}^{n_{x}} \delta \boldsymbol{v}_{a} N_{a}^{\boldsymbol{x}} ; \quad \delta \boldsymbol{F}=\sum_{a=1}^{n_{F}} \delta \boldsymbol{F}_{a} N_{a}^{\boldsymbol{F}} ; \quad \ldots ; \quad \delta \boldsymbol{\Sigma}_{\boldsymbol{F}}=\sum_{a=1}^{n_{F}} \delta \boldsymbol{\Sigma}_{\boldsymbol{F}}^{\boldsymbol{a}} N_{a}^{\boldsymbol{\Sigma}_{\boldsymbol{F}}} \\
\boldsymbol{u}=\sum_{a=1}^{n_{x}} \boldsymbol{u}_{a} N_{a}^{\boldsymbol{x}} ; \quad \Delta \boldsymbol{F}=\sum_{a=1}^{n_{F}} \Delta \boldsymbol{F}_{a} N_{a}^{\boldsymbol{F}} ; \quad \ldots ; \quad \Delta \boldsymbol{\Sigma}_{\boldsymbol{F}}=\sum_{a=1}^{n_{F}} \Delta \boldsymbol{\Sigma}_{\boldsymbol{F}}^{\boldsymbol{a}} N_{a}^{\boldsymbol{\Sigma}_{\boldsymbol{F}}}
\end{gathered}
$$

Finite element equations are derived by simply substituting the above expressions into the functional expressions provided in the previous section. 
In many cases this is a rather standard process and leads to well established equations. For instance, substituting the above interpolation for the virtual velocity into any of the virtual work statements given above by equations (84), (92), (106) or (112) leads to the standard definition of the residual forces as:

$D_{1} \Pi[\delta \boldsymbol{v}]=\sum_{a} \boldsymbol{R}_{\boldsymbol{x}}^{a} \cdot \delta \boldsymbol{x} ; \quad \boldsymbol{R}_{\boldsymbol{x}}^{a}=\int_{V} \boldsymbol{P} \nabla_{0} N_{a}^{\boldsymbol{x}} d V-\int_{V} \boldsymbol{f}_{0} N_{a}^{\boldsymbol{x}} d V-\int_{\partial_{t} V} \boldsymbol{t}_{0} N_{a}^{\boldsymbol{x}} d A$

where the Piola-Kirchhoff stress above will be evaluated in accordance with each of the formulations presented in the previous section. Similar expressions for other residual terms can be easily derived. For instance, the geometric compatibility residuals emerge from the discretisation of equations (95) or (107) as:

$$
\begin{gathered}
\boldsymbol{R}_{\Sigma_{\boldsymbol{F}}}^{a}=\int_{V}\left(\boldsymbol{F}_{x}-\boldsymbol{F}\right) N_{a}^{\boldsymbol{F}} d V ; \quad \boldsymbol{R}_{\Sigma_{\boldsymbol{H}}}^{a}=\int_{V}\left(\boldsymbol{H}_{x}-\boldsymbol{H}\right) N_{a}^{\boldsymbol{H}} d V ; \\
\boldsymbol{R}_{\Sigma_{J}}^{a}=\int_{V}\left(J_{x}-J\right) N_{a}^{J} d V .
\end{gathered}
$$

Very similar equations emerge in the case of the mixed potential for the constitutive equation residuals:

$$
\begin{gathered}
\boldsymbol{R}_{\boldsymbol{F}}^{a}=\int_{V}\left(\frac{\partial W}{\partial \boldsymbol{F}}-\boldsymbol{\Sigma}_{\boldsymbol{F}}\right) N_{a}^{\boldsymbol{F}} d V ; \quad \boldsymbol{R}_{\boldsymbol{H}}^{a}=\int_{V}\left(\frac{\partial W}{\partial \boldsymbol{H}}-\boldsymbol{\Sigma}_{\boldsymbol{H}}\right) N_{a}^{\boldsymbol{H}} d V ; \\
\boldsymbol{R}_{J}^{a}=\int_{V}\left(\frac{\partial W}{\partial J}-\Sigma_{J}\right) N_{a}^{J} d V .
\end{gathered}
$$

In order to complete the finite element formulation it is necessary to derive equations for the components of the tangent matrix by discretising the tangent operators defined in the previous section. For the case of the mixed formulation, the resulting matrix operator can be represented as:

$$
D^{2} \Pi_{M}[\delta \boldsymbol{v}, \delta \boldsymbol{D}, \delta \boldsymbol{\Sigma} ; \boldsymbol{u}, \Delta \boldsymbol{D}, \Delta \boldsymbol{\Sigma}]=\left[\begin{array}{lll}
\delta \boldsymbol{v} & \delta \boldsymbol{D} & \delta \boldsymbol{\Sigma}
\end{array}\right]\left[\begin{array}{ccc}
\boldsymbol{K}_{\boldsymbol{x} \boldsymbol{x}} & \mathbf{0} & \boldsymbol{K}_{\boldsymbol{x} \boldsymbol{\Sigma}} \\
\mathbf{0} & \boldsymbol{K}_{\boldsymbol{D} \boldsymbol{D}} & \boldsymbol{K}_{\boldsymbol{D} \boldsymbol{\Sigma}} \\
\boldsymbol{K}_{\boldsymbol{\Sigma} \boldsymbol{x}} & \boldsymbol{K}_{\boldsymbol{L} \boldsymbol{D}} & \mathbf{0}
\end{array}\right]\left[\begin{array}{c}
\boldsymbol{u} \\
\Delta \boldsymbol{D} \\
\Delta \boldsymbol{\Sigma}
\end{array}\right]
$$


where $\boldsymbol{D}=\left\{\boldsymbol{F}_{a}, \boldsymbol{H}_{a}, J_{a}\right\}$ denotes the set of strain variables and $\boldsymbol{\Sigma}=$ $\left\{\boldsymbol{\Sigma}_{\boldsymbol{F}_{a}}, \boldsymbol{\Sigma}_{\boldsymbol{H}_{a}}, \Sigma_{J_{a}}\right\}$ denotes the set of stress conjugate variables. Alternatively, for the case of a complementary energy potential the equivalent expression becomes:

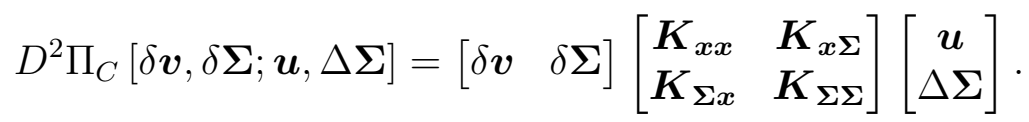

Some of the terms in the above matrices are very straightforward to obtain. For instance the term $\boldsymbol{K}_{\boldsymbol{D} \boldsymbol{\Sigma}}$ relating stresses and strains in equation (127) follows from the discretisation of the corresponding tangent operator component given in equation (101) as:

$$
\boldsymbol{K}_{\boldsymbol{D} \boldsymbol{\Sigma} \boldsymbol{a}}^{a b}=-\int_{V}\left[\begin{array}{ccc}
N_{a}^{\boldsymbol{F}} N_{b}^{\boldsymbol{F}} \boldsymbol{\mathcal { I }} & \mathbf{0} & \mathbf{0} \\
\mathbf{0} & N_{a}^{\boldsymbol{H}} N_{b}^{\boldsymbol{H}} \mathcal{I} & \mathbf{0} \\
\mathbf{0} & \mathbf{0} & N_{a}^{J} N_{b}^{J} \mathcal{I}
\end{array}\right] d V
$$

where $\mathcal{I}$ denotes the components of the fourth order identity tensor.

The diagonal components of the tangent matrix relating associated with strains or stresses, $\boldsymbol{K}_{\boldsymbol{D} \boldsymbol{D}}$ and $\boldsymbol{K}_{\boldsymbol{\Sigma} \boldsymbol{\Sigma}}$ respectively, can be obtained from the discretisation of the corresponding Hessian term in the tangent operator. For instance, the strain term becomes:

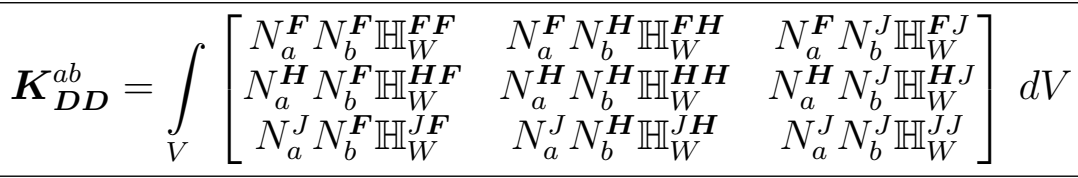

And the corresponding tangent stress term:

$$
\boldsymbol{K}_{\boldsymbol{\Sigma} \boldsymbol{\Sigma}}^{a b}=-\int_{V}\left[\begin{array}{ccc}
N_{a}^{\boldsymbol{F}} N_{b}^{\boldsymbol{F}} \mathbb{H}_{\Upsilon}^{\boldsymbol{\Sigma}_{\boldsymbol{F}} \boldsymbol{\Sigma}_{\boldsymbol{F}}} & N_{a}^{\boldsymbol{F}} N_{b}^{\boldsymbol{H}} \mathbb{H}_{\Upsilon}^{\boldsymbol{\Sigma}_{\boldsymbol{F}} \boldsymbol{\Sigma}_{\boldsymbol{H}}} & N_{a}^{\boldsymbol{F}} N_{b}^{J} \mathbb{H}_{\Upsilon}^{\boldsymbol{\Sigma}_{\boldsymbol{F}} \Sigma_{J}} \\
N_{a}^{\boldsymbol{H}} N_{b}^{\boldsymbol{F}} \mathbb{H}_{\Upsilon}^{\boldsymbol{\Sigma}_{\boldsymbol{H}} \boldsymbol{\Sigma} \boldsymbol{\Sigma}_{\boldsymbol{F}}} & N_{a}^{\boldsymbol{H}} N_{b}^{\boldsymbol{H}} \mathbb{H}_{\Upsilon}^{\boldsymbol{\Sigma}_{H} \boldsymbol{\Sigma}_{\boldsymbol{H}}} & N_{a}^{\boldsymbol{H}} N_{b}^{J} \mathbb{H}_{\Upsilon}^{\boldsymbol{\Sigma}_{\boldsymbol{H}} \Sigma_{J}} \\
N_{a}^{J} N_{b}^{\boldsymbol{F}} \mathbb{H}_{\Upsilon}^{\Sigma_{J} \boldsymbol{\Sigma}_{\boldsymbol{F}}} & N_{a}^{J} N_{b}^{\boldsymbol{H}} \mathbb{H}_{\Upsilon}^{\Sigma_{J} \boldsymbol{\Sigma}_{\boldsymbol{H}}} & N_{a}^{J} N_{b}^{J} \mathbb{H}_{\Upsilon}^{\Sigma_{J} \Sigma_{J}}
\end{array}\right] d V
$$

The final two terms of the tangent matrix involve derivatives with respect to geometry and require more careful analysis. Consider first the top diagonal component $\boldsymbol{K}_{\boldsymbol{x} \boldsymbol{x}}$. This term emerges from the discretisation of the initial stress component of the tangent operator defined in equation (99). After 
discretisation and some simple algebra using property (16) of the tensor cross product, the component relating nodes $a, b$ of this operator becomes:

$$
\begin{aligned}
D_{1,1}^{2} \Pi_{M}\left[\delta \boldsymbol{v}_{a} N_{a}^{\boldsymbol{x}} ; \boldsymbol{u}_{b} N_{b}^{\boldsymbol{x}}\right] & =\int_{V}\left(\boldsymbol{\Sigma}_{\boldsymbol{H}}+\Sigma_{J} \boldsymbol{F}_{\boldsymbol{x}}\right):\left[\left(\delta \boldsymbol{v}_{a} \otimes \boldsymbol{\nabla}_{0} N_{a}^{\boldsymbol{x}}\right) \times\left(\boldsymbol{u}_{b} \otimes \boldsymbol{\nabla}_{0} N_{b}^{\boldsymbol{x}}\right)\right] d V \\
& =\int_{V}\left(\boldsymbol{\Sigma}_{\boldsymbol{H}}+\Sigma_{J} \boldsymbol{F}_{\boldsymbol{x}}\right):\left[\left(\delta \boldsymbol{v}_{a} \times \boldsymbol{u}_{b}\right) \otimes\left(\boldsymbol{\nabla}_{0} N_{a}^{\boldsymbol{x}} \times \boldsymbol{\nabla}_{0} N_{b}^{\boldsymbol{x}}\right)\right] d V \\
& =\left(\delta \boldsymbol{v}_{a} \times \boldsymbol{u}_{b}\right) \cdot \int_{V}\left(\boldsymbol{\Sigma}_{\boldsymbol{H}}+\Sigma_{J} \boldsymbol{F}_{\boldsymbol{x}}\right)\left(\boldsymbol{\nabla}_{0} N_{a}^{\boldsymbol{x}} \times \boldsymbol{\nabla}_{0} N_{b}^{\boldsymbol{x}}\right) d V \\
& =\left(\delta \boldsymbol{v}_{a} \times \boldsymbol{u}_{b}\right) \cdot \boldsymbol{k}_{\boldsymbol{x} \boldsymbol{x}}^{a b} \\
& =\delta \boldsymbol{v}_{a} \cdot \boldsymbol{K}_{\boldsymbol{x} \boldsymbol{x}}^{a b} \boldsymbol{u}_{b}
\end{aligned}
$$

where the initial stress vector $\boldsymbol{k}_{\boldsymbol{x} \boldsymbol{x}}^{a b}$ and equivalent skew symmetric matrix $\boldsymbol{K}_{\boldsymbol{x} \boldsymbol{x}}^{a b}$ are:

$$
\begin{aligned}
& \boldsymbol{k}_{\boldsymbol{x} \boldsymbol{x}}^{a b}=\int_{V}\left(\boldsymbol{\Sigma}_{\boldsymbol{H}}+\Sigma_{J} \boldsymbol{F}_{\boldsymbol{x}}\right)\left(\boldsymbol{\nabla}_{0} N_{a}^{\boldsymbol{x}} \times \boldsymbol{\nabla}_{0} N_{b}^{\boldsymbol{x}}\right) d V \\
& {\left[\boldsymbol{K}_{\boldsymbol{x} \boldsymbol{x}}^{a b}\right]_{i j}=\mathcal{E}_{i j k}\left[\boldsymbol{k}_{\boldsymbol{x} \boldsymbol{x}}^{a b}\right]_{k}}
\end{aligned}
$$

Similar derivations starting from equation (102) yield the final component relating geometry changes to stress changes $\boldsymbol{K}_{\boldsymbol{x} \boldsymbol{\Sigma}}$. For clarity, however, the terms relating to each one of the conjugate stresses is derived individually. The first term relating geometry to conjugate stress $\boldsymbol{\Sigma}_{\boldsymbol{F}}$ is obtained as:

$$
\begin{aligned}
D_{\boldsymbol{x} ; \boldsymbol{\Sigma}_{\boldsymbol{F}}}^{2} \Pi_{M}\left[\delta \boldsymbol{v}_{a} N_{a}^{\boldsymbol{x}} ; \Delta \boldsymbol{\Sigma}_{\boldsymbol{F}}^{b} N_{b}^{\boldsymbol{F}}\right] & =\int_{V}\left(\delta \boldsymbol{v}_{a} \otimes \boldsymbol{\nabla}_{0} N_{a}^{\boldsymbol{x}}\right): \Delta \boldsymbol{\Sigma}_{\boldsymbol{F}}^{b} N_{b}^{\boldsymbol{F}} d V \\
& =\delta \boldsymbol{v}_{a} \cdot\left[\int_{V}\left(\boldsymbol{I} \otimes \boldsymbol{\nabla}_{0} N_{a}^{\boldsymbol{x}}\right) N_{b}^{\boldsymbol{F}} d V\right]: \Delta \boldsymbol{\Sigma}_{\boldsymbol{F}}^{b} \\
& =\delta \boldsymbol{v}_{a} \cdot \mathcal{K}_{\boldsymbol{x} \boldsymbol{\Sigma}_{\boldsymbol{F}}}^{a b}: \Delta \boldsymbol{\Sigma}_{\boldsymbol{F}}^{b}
\end{aligned}
$$

where the third order tensor $\mathcal{K}_{\boldsymbol{x} \boldsymbol{\Sigma}_{\boldsymbol{F}}}^{a b}$ is:

$$
\mathcal{K}_{\boldsymbol{x} \boldsymbol{\Sigma}_{\boldsymbol{F}}}^{a b}=\int_{V}\left(\boldsymbol{I} \otimes \boldsymbol{\nabla}_{0} N_{a}^{\boldsymbol{x}}\right) N_{b}^{\boldsymbol{F}} d V
$$


The third term relating geometry to conjugate stress $\Sigma_{J}$ is obtained as:

$$
\begin{aligned}
D_{\boldsymbol{x} ; \Sigma_{J}}^{2} \Pi_{M}\left[\delta \boldsymbol{v}_{a} N_{a}^{\boldsymbol{x}} ; \Delta \Sigma_{J}^{b} N_{b}^{J}\right] & =\int_{V} \Delta \Sigma_{J}^{b} N_{b}^{J} \boldsymbol{H}_{\boldsymbol{x}}:\left(\delta \boldsymbol{v}_{a} \otimes \boldsymbol{\nabla}_{0} N_{a}^{\boldsymbol{x}}\right) d V \\
& =\delta \boldsymbol{v}_{a} \cdot\left[\int_{V} N_{b}^{J} \boldsymbol{H}_{\boldsymbol{x}} \boldsymbol{\nabla}_{0} N_{a}^{\boldsymbol{x}} d V\right] \Delta \Sigma_{J}^{b} \\
& =\delta \boldsymbol{v}_{a} \cdot \boldsymbol{k}_{\boldsymbol{x} \Sigma_{J}}^{a b} \Delta \Sigma_{J}^{b}
\end{aligned}
$$

where the vector $\boldsymbol{k}_{\boldsymbol{x} \Sigma_{J}}^{a b}$ is:

$$
\boldsymbol{k}_{\boldsymbol{x} \Sigma_{J}}^{a b}=\int_{V} N_{b}^{J} \boldsymbol{H}_{\boldsymbol{x}} \boldsymbol{\nabla}_{0} N_{a}^{\boldsymbol{x}} d V
$$

Finally, the term relating geometry to changes in $\boldsymbol{\Sigma}_{\boldsymbol{H}}$ is:

$$
\begin{aligned}
D_{\boldsymbol{x} ; \boldsymbol{\Sigma}_{\boldsymbol{H}}}^{2} \Pi_{M}\left[\delta \boldsymbol{v}_{a} N_{a}^{\boldsymbol{x}} ; \Delta \boldsymbol{\Sigma}_{\boldsymbol{H}}^{b} N_{b}^{\boldsymbol{H}}\right] & =\int_{V}\left[\boldsymbol{F}_{\boldsymbol{x}} \times\left(\delta \boldsymbol{v}_{a} \otimes \boldsymbol{\nabla}_{0} N_{a}^{\boldsymbol{x}}\right)\right]: \Delta \boldsymbol{\Sigma}_{\boldsymbol{H}}^{b} N_{b}^{\boldsymbol{H}} d V \\
& =-\delta \boldsymbol{v}_{a} \times\left[\int_{V}\left(\boldsymbol{F}_{\boldsymbol{x}} \times \nabla_{0} N_{a}^{\boldsymbol{x}}\right) N_{b}^{\boldsymbol{H}} d V\right]: \Delta \boldsymbol{\Sigma}_{\boldsymbol{H}}^{b} \\
& =\delta \boldsymbol{v}_{a} \cdot \mathcal{K}_{\boldsymbol{x} \boldsymbol{\Sigma}_{\boldsymbol{H}}}^{a b}: \Delta \boldsymbol{\Sigma}_{\boldsymbol{H}}^{b}
\end{aligned}
$$

with:

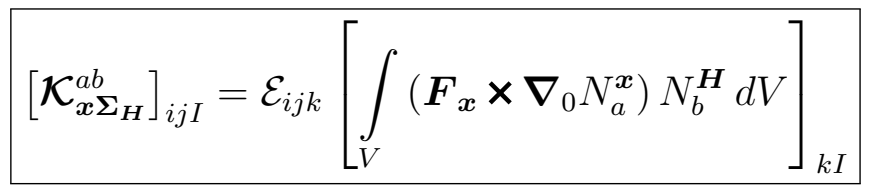

\subsection{Complementary energy application case}

The equations provided above can be implemented using a variety of finite element spaces. Of course, not all choices will lead to effective or valid finite element formulations. Moreover, the cost of implementation of mixed formulation may be significantly higher than that of standard displacement based approaches given the number of additional unknowns created. However, careful analysis of the continuity required for each of the variables, shows that only displacements need to be continuous across elements. Stress and strain 
variables can actually be discretised independently on each element of the mesh. This enables a static condensation process to be carried out before assembly of the global tangent matrix. In order to illustrate this process and as an example of the complementary energy formulation proposed, this section describes a choice of interpolation functions that permits such a condensation. In particular, a quadratic tetrahedron element is proposed for the geometry discretisation, with linear element by element interpolations for the stresses conjugate to the deformation gradient and its co-factor and a constant interpolation for the stress conjugate to the Jacobian. The resulting element is very similar to that proposed in reference [24].

The resulting formulation is obtained by applying the set of equations derived in the previous section with shape functions $N_{a}^{\boldsymbol{x}}(a=1, \ldots, 10)$ that are quadratic and continuous across elements; $N_{a}^{\boldsymbol{F}}=N_{a}^{\boldsymbol{H}}(a=1, \ldots, 4)$ linear and discontinuous across elements and $N_{a}^{J}(a=1)$ constant and discontinuous across elements. The fact that the shape functions associated with stresses are discontinuous across elements allows for the elimination of stress unknowns inside each element to take place. In order to illustrate this, note that for a given element the system of equations to be solved can be written as:

$$
\left[\begin{array}{ll}
\boldsymbol{K}_{\boldsymbol{x} \boldsymbol{x}}^{e} & \boldsymbol{K}_{\boldsymbol{x} \boldsymbol{\Sigma}}^{e} \\
\boldsymbol{K}_{\boldsymbol{\Sigma} \boldsymbol{x}}^{e} & \boldsymbol{K}_{\boldsymbol{\Sigma} \boldsymbol{\Sigma}}^{e}
\end{array}\right]\left[\begin{array}{c}
\boldsymbol{u}^{e} \\
\Delta \boldsymbol{\Sigma}^{e}
\end{array}\right]=-\left[\begin{array}{l}
\boldsymbol{R}_{\boldsymbol{x}}^{e} \\
\boldsymbol{R}_{\Sigma}^{e}
\end{array}\right]
$$

The second row of equations enables the stress increments to be expressed in terms of displacements as:

$$
\Delta \boldsymbol{\Sigma}^{e}=-\left[\boldsymbol{K}_{\boldsymbol{\Sigma} \boldsymbol{\Sigma}}^{e}\right]^{-1}\left(\boldsymbol{K}_{\boldsymbol{\Sigma} \boldsymbol{x}}^{e} \boldsymbol{u}^{e}+\boldsymbol{R}_{\boldsymbol{\Sigma}}^{e}\right) .
$$

Substituting this relationship into the first row of equation (140) gives an augmented set of equations for the displacement vector:

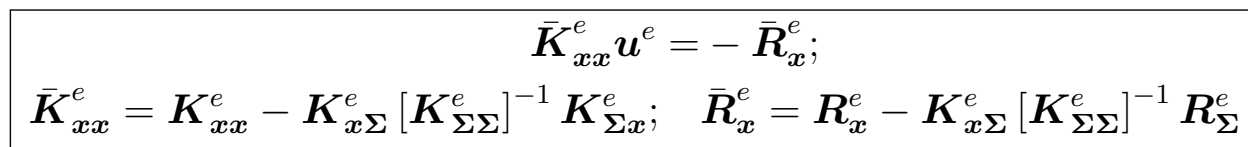

These augmented stiffness matrix and residual vector can now be assembled into a global system in the usual finite element manner.

\subsection{Stabilised linear tetrahedron for incompressible elasticity}

As a final case study of the application of the present framework, a linear tetrahedron for modelling full incompressible elasticity is described. This 
will also serve as an example of the introduction of Petrov-Galerkin type of stabilisation to a mixed formulation in which the discretisation spaces do not satisfy the LBB condition.

In the proposed formulation, both the geometry and pressure are discretised using linear tetrahedral elements in terms of the same standard shape functions $N_{a}$ as:

$$
\boldsymbol{x}=\sum_{a=1}^{4} \boldsymbol{x}_{a} N_{a} ; \quad p=\sum_{a=1}^{4} p_{a} N_{a} .
$$

Note that in this case the pressure is continuous across elements and therefore cannot be eliminated locally. This adds to the number of global degrees of freedom but the resulting tetrahedron will be capable of simulating incompressible deformations without locking [47]. Unfortunately, it is well know that an incompressible mixed formulation with linear displacements and linear pressures on tetrahedral element spaces does not satisfy the necessary LBB condition and leads to unstable solutions [3]. The classical solution to this problem is to introduce Petrov-Galerkin stabilisation [46-49]. In particular, this is done here by defining the stabilised virtual pressures and velocities as:

$$
\begin{aligned}
\delta p^{s t} & =\delta p-\tau_{p}\left(\boldsymbol{H}_{\boldsymbol{x}}: \boldsymbol{\nabla}_{0} \delta \boldsymbol{v}\right) \\
\delta \boldsymbol{v}^{s t} & =\delta \boldsymbol{v}-\tau_{\boldsymbol{v}}\left(\boldsymbol{H}_{\boldsymbol{x}} \boldsymbol{\nabla}_{0} \delta p\right)
\end{aligned}
$$

Note that in the usual Petrov-Galerkin manner, the term in parenthesis in (144a) is the derivative of the constraint equation $\operatorname{det} \boldsymbol{\nabla}_{0} \boldsymbol{x}=1$ and that $\tau_{p}$ has the same units as an elastic constant. The stabilised term in (144b) is defined following [46], namely $\tau_{\boldsymbol{v}}=\frac{\alpha h^{2}}{2 \mu}$, where $\mu$ is an elastic constant, $h$ is the mesh size and $\alpha$ is a non-dimensional stabilisation parameter. Substituting expression (144a) into the constraint equation (116) leads to an additional term in the principle of virtual work (112) as:

$$
D_{1} \hat{\Pi}_{M}^{s t}[\delta \boldsymbol{v}]=\int_{V} \boldsymbol{P}_{s t}: \nabla_{0} \delta \boldsymbol{v} d V-\int_{V} \boldsymbol{f}_{0} \cdot \delta \boldsymbol{v} d V-\int_{\partial_{t} V} \boldsymbol{t}_{0} \cdot \delta \boldsymbol{v} d A=0
$$

where the stabilised first Piola-Kirchhoff tensor is:

$$
\boldsymbol{P}_{s t}=\Sigma_{\boldsymbol{F}}^{\boldsymbol{x}}+\boldsymbol{\Sigma}_{\boldsymbol{H}}^{\boldsymbol{x}} \times \boldsymbol{F}_{\boldsymbol{x}}+\Sigma_{J}^{s t} \boldsymbol{H}_{\boldsymbol{x}} ; \quad \Sigma_{J}^{s t}=\hat{\Sigma}_{\boldsymbol{J}}^{\boldsymbol{x}}+p+\tau_{p}\left(J_{\boldsymbol{x}}-1\right) .
$$


Substituting equation (144b) into the principle of virtual work (112) leads to an additional term in the constraint equation (116),

$$
D_{2} \hat{\Pi}_{M}^{s t}[\delta p]=\int_{V} \delta p\left(J_{\boldsymbol{x}}-1\right) d V-\int_{V} \tau_{\boldsymbol{v}}\left(\boldsymbol{H}_{\boldsymbol{x}} \boldsymbol{\nabla}_{0} \delta p\right) \cdot\left(\boldsymbol{H}_{\boldsymbol{x}} \boldsymbol{\nabla}_{0} p\right) d V .
$$

Clearly, as the mesh is refined and the enforcement of the volumetric constraint is improved, the stabilisation terms in (146) and (147) will tend to zero. Introducing the discretisation equations for the geometry and pressure gives the equilibrium and constraints residuals as:

$$
\begin{aligned}
\boldsymbol{R}_{\boldsymbol{x}}^{a} & =\int_{V} \boldsymbol{P}_{s t} \boldsymbol{\nabla}_{0} N_{a} d V-\int_{V} \boldsymbol{f}_{0} N_{a}^{\boldsymbol{x}} d V-\int_{\partial_{t} V} \boldsymbol{t}_{0} N_{a}^{\boldsymbol{x}} d A \\
R_{p}^{a} & =\int_{V}\left(J_{\boldsymbol{x}}-1\right) N_{a} d v-\int_{V} \tau_{\boldsymbol{v}}\left(\boldsymbol{H}_{\boldsymbol{x}} \boldsymbol{\nabla}_{0} N_{a}\right) \cdot\left(\boldsymbol{H}_{\boldsymbol{x}} \boldsymbol{\nabla}_{0} p\right) d V .
\end{aligned}
$$

The system of liner equations to be solved at each Newton-Raphson iteration is now expressed globally as:

$$
\left[\begin{array}{cc}
\boldsymbol{K}_{\boldsymbol{x} \boldsymbol{x}}^{s t} & \boldsymbol{k}_{\boldsymbol{x} p} \\
\boldsymbol{k}_{p \boldsymbol{x}}^{s t} & \boldsymbol{k}_{p p}^{s t}
\end{array}\right]\left[\begin{array}{c}
\boldsymbol{u} \\
\Delta \boldsymbol{p}
\end{array}\right]=-\left[\begin{array}{c}
\boldsymbol{R}_{\boldsymbol{x}} \\
\boldsymbol{R}_{p}
\end{array}\right]
$$

The off-diagonal term $\boldsymbol{k}_{\boldsymbol{x} p}$ of (149) is in fact identical to that obtained in the previous section relating the volumetric stress and geometry via equation (137), that is:

$$
\boldsymbol{k}_{\boldsymbol{x} p}^{a b}=\int_{V} N_{b} \boldsymbol{H}_{\boldsymbol{x}} \nabla_{0} N_{a} d V
$$

The diagonal component $\boldsymbol{K}_{\boldsymbol{x} \boldsymbol{x}}^{\text {st }}$ in the above tangent matrix can be broken down into three terms: a constitutive component due to the Hessian of the isochoric strain energy $\hat{W}$, an initial stress term similar to that derived in equation (133) plus a contribution from geometric derivative of the stabilisation term $\tau_{p}\left(J_{\boldsymbol{x}}-1\right)$, that is:

$$
\boldsymbol{K}_{\boldsymbol{x} \boldsymbol{x}}^{s t}=\left(\boldsymbol{K}_{\hat{W}}+\boldsymbol{K}_{0}+\boldsymbol{K}_{\tau_{p}}\right) .
$$


The stabilisation component is easily evaluated for equations (145) and (146) as:

$$
\begin{aligned}
\delta \boldsymbol{v}_{a} \cdot \boldsymbol{K}_{\tau_{p}}^{a b} \boldsymbol{u}_{b} & =\int_{V} \tau_{p}\left[\boldsymbol{H}_{\boldsymbol{x}}:\left(\delta \boldsymbol{v}_{a} \otimes \boldsymbol{\nabla}_{0} N_{a}\right)\right] D J_{\boldsymbol{x}}\left[\boldsymbol{u}_{b} N_{b}\right] d V \\
& =\int_{V} \tau_{p}\left[\boldsymbol{H}_{\boldsymbol{x}}:\left(\delta \boldsymbol{v}_{a} \otimes \boldsymbol{\nabla}_{0} N_{a}\right)\right]\left[\boldsymbol{H}_{\boldsymbol{x}}:\left(\boldsymbol{u}_{b} \otimes \boldsymbol{\nabla}_{0} N_{b}\right)\right] d V \\
& =\int_{V} \tau_{p}\left(\delta \boldsymbol{v}_{a} \cdot \boldsymbol{H}_{\boldsymbol{x}} \boldsymbol{\nabla}_{0} N_{a}\right)\left(\boldsymbol{u}_{b} \cdot \boldsymbol{H}_{\boldsymbol{x}} \boldsymbol{\nabla}_{0} N_{b}\right) d V \\
& =\delta \boldsymbol{v}_{a} \cdot\left[\int_{V} \tau_{p}\left(\boldsymbol{H}_{\boldsymbol{x}} \boldsymbol{\nabla}_{0} N_{a}\right) \otimes\left(\boldsymbol{H}_{\boldsymbol{x}} \boldsymbol{\nabla}_{0} N_{b}\right) d V\right] \boldsymbol{u}_{b}
\end{aligned}
$$

which gives:

$$
\boldsymbol{K}_{\tau_{p}}^{a b}=\int_{V} \tau_{p}\left(\boldsymbol{H}_{\boldsymbol{x}} \boldsymbol{\nabla}_{0} N_{a}\right) \otimes\left(\boldsymbol{H}_{\boldsymbol{x}} \boldsymbol{\nabla}_{0} N_{b}\right) d V
$$

The initial stress component is given by equation (133) with a volumetric stress component that includes the additional stabilisation term, that is:

$$
\begin{aligned}
& \boldsymbol{k}_{0}^{a b}=\int_{V}\left(\boldsymbol{\Sigma}_{\boldsymbol{H}}+\Sigma_{J} \boldsymbol{F}_{x}\right)\left(\boldsymbol{\nabla}_{0} N_{a} \times \boldsymbol{\nabla}_{0} N_{b}\right) d V \\
& {\left[\boldsymbol{K}_{0}^{a b}\right]_{i j}=\mathcal{E}_{i j k}\left[\boldsymbol{k}_{0}^{a b}\right]_{k}}
\end{aligned}
$$

Finally, the constitutive component is given by the Hessian of the isochoric strain energy as:

$$
\delta \boldsymbol{v}_{a} \cdot \boldsymbol{K}_{\hat{W}}^{a b} \boldsymbol{u}_{b}=\int_{V}\left[D \boldsymbol{F}_{\boldsymbol{x}}\left[\delta \boldsymbol{v}_{a} N_{a}\right]: \quad D \boldsymbol{H}_{\boldsymbol{x}}\left[\delta \boldsymbol{v}_{a} N_{a}\right]: \quad D J_{\boldsymbol{x}}\left[\delta \boldsymbol{v}_{a} N_{a}\right]\right]\left[\mathbb{H}_{\Upsilon}\right]\left[\begin{array}{c}
: D \boldsymbol{F}_{\boldsymbol{x}}\left[\boldsymbol{u}_{b} N_{b}\right] \\
: D \boldsymbol{H}_{\boldsymbol{x}}\left[\boldsymbol{u}_{b} N_{b}\right] \\
D J_{\boldsymbol{x}}\left[\boldsymbol{u}_{b} N_{b}\right]
\end{array}\right] d V
$$

The off-diagonal term $\boldsymbol{k}_{p \boldsymbol{x}}^{s t}$ in (149) can be broken down into three terms stemming from the linearisation of the right hand side of equation (147) with respect to the geometry, that is: 


$$
\boldsymbol{k}_{p \boldsymbol{x}}^{s t}=\boldsymbol{k}_{J}-\boldsymbol{k}_{\boldsymbol{H}_{1}}-\boldsymbol{k}_{\boldsymbol{H}_{2}}
$$

where

$$
\delta p_{a} \boldsymbol{k}_{J}^{a b} \cdot \boldsymbol{u}_{b}=\int_{V} \delta p_{a} N_{a} D J_{\boldsymbol{x}}\left[\boldsymbol{u}_{b} N_{b}\right] d V=\delta p_{a}\left[\int_{V} N_{a} \boldsymbol{H}_{\boldsymbol{x}} \boldsymbol{\nabla}_{0} N_{b} d V\right] \boldsymbol{u}_{b}
$$

which gives

$$
\boldsymbol{k}_{J}^{a b}=\int_{V} N_{a} \boldsymbol{H}_{\boldsymbol{x}} \boldsymbol{\nabla}_{0} N_{b} d V
$$

Computation of the directional derivative of the second term on the right hand side of equation (147) and use of properties (7) and (11) yields:

$$
\begin{aligned}
\delta p_{a} \boldsymbol{k}_{\boldsymbol{H}_{1}}^{a b} \cdot \boldsymbol{u}_{b} & =\delta p_{a} \int_{V} \tau_{\boldsymbol{v}}\left(\boldsymbol{H}_{\boldsymbol{x}} \boldsymbol{\nabla}_{0} N_{a}\right) \cdot\left(D \boldsymbol{H}_{\boldsymbol{x}}\left[\boldsymbol{u}_{b} N_{b}\right] \boldsymbol{\nabla}_{0} p\right) d V \\
& =\delta p_{a} \int_{V} \tau_{\boldsymbol{v}}\left(\boldsymbol{H}_{\boldsymbol{x}} \boldsymbol{\nabla}_{0} N_{a}\right) \cdot\left[\left(\boldsymbol{F}_{\boldsymbol{x}} \times\left(\boldsymbol{u}_{b} \otimes \boldsymbol{\nabla}_{0} N_{b}\right)\right) \boldsymbol{\nabla}_{0} p\right] d V \\
& =\delta p_{a} \int_{V} \tau_{\boldsymbol{v}}\left[\left(\boldsymbol{H}_{\boldsymbol{x}} \boldsymbol{\nabla}_{0} N_{a} \otimes \boldsymbol{\nabla}_{0} p\right) \times \boldsymbol{F}_{\boldsymbol{x}}\right]:\left(\boldsymbol{u}_{b} \otimes \boldsymbol{\nabla}_{0} N_{b}\right) d V \\
& =\delta p_{a}\left[\int_{V} \tau_{\boldsymbol{v}}\left[\left(\boldsymbol{H}_{\boldsymbol{x}} \boldsymbol{\nabla}_{0} N_{a} \otimes \boldsymbol{\nabla}_{0} p\right) \times \boldsymbol{F}_{\boldsymbol{x}}\right] \boldsymbol{\nabla}_{0} N_{b} d V\right] \cdot \boldsymbol{u}_{b}
\end{aligned}
$$

which gives

$$
\boldsymbol{k}_{\boldsymbol{H}_{1}}^{a b}=\int_{V} \tau_{\boldsymbol{v}}\left[\left(\boldsymbol{H}_{\boldsymbol{x}} \boldsymbol{\nabla}_{0} N_{a} \otimes \boldsymbol{\nabla}_{0} p\right) \times \boldsymbol{F}_{\boldsymbol{x}}\right] \boldsymbol{\nabla}_{0} N_{b} d V
$$

Analogously,

$$
\boldsymbol{k}_{\boldsymbol{H}_{2}}^{a b}=\int_{V} \tau_{\boldsymbol{v}}\left[\left(\boldsymbol{H}_{\boldsymbol{x}} \boldsymbol{\nabla}_{0} p \otimes \boldsymbol{\nabla}_{0} N_{a}\right) \times \boldsymbol{F}_{\boldsymbol{x}}\right] \boldsymbol{\nabla}_{0} N_{b} d V
$$


Finally, the diagonal term $\boldsymbol{k}_{p p}^{s t}$ is given as,

$$
k_{p p}^{s t, a b}=-\int_{V} \tau_{\boldsymbol{v}}\left(\boldsymbol{H}_{\boldsymbol{x}} \boldsymbol{\nabla}_{0} N_{a}\right) \cdot\left(\boldsymbol{H}_{\boldsymbol{x}} \boldsymbol{\nabla}_{0} N_{b}\right) d V
$$

\section{Numerical examples}

The objective of this section is to present a series of numerical examples in order to prove the robustness, accuracy and applicability of the computational framework presented above. Numerical results dealing with both compressible and incompressible polyconvex constitutive models will be presented.

For the compressible case, four different formulations are presented: i) a standard displacement $\{\boldsymbol{x}\}$ based formulation as described in Section 4.1, hereby denoted as DF; ii) a seven field $\left\{\boldsymbol{x}, \boldsymbol{F}, \boldsymbol{H}, J, \boldsymbol{\Sigma}_{\boldsymbol{F}}, \boldsymbol{\Sigma}_{\boldsymbol{H}}, \Sigma_{J}\right\}$ mixed formulation as described in Section 4.2, denoted as $\mathbf{M 7 F}$; iii) a four field $\left\{\boldsymbol{x}, \boldsymbol{\Sigma}_{\boldsymbol{F}}, \boldsymbol{\Sigma}_{\boldsymbol{H}}, \Sigma_{J}\right\}$ mixed formulation based on the complementary energy principle described in Section 4.3, denoted by MCF and iv) an alternative five field $\left\{\boldsymbol{x}, \boldsymbol{H}, J, \boldsymbol{\Sigma}_{\boldsymbol{H}}, \Sigma_{J}\right\}$ mixed formulation, denoted as $\mathbf{M 5 F}$. In this last case, the deformation gradient is enforced to coincide strongly with the material gradient of the spatial coordinates, namely $\boldsymbol{F}=\boldsymbol{\nabla}_{0} \boldsymbol{x}$.

All of the numerical results presented correspond to the following selection of functional spaces: continuous quadratic interpolation of the displacement field (geometry) $\boldsymbol{x}$, piecewise linear interpolation of the strain and stress fields $\boldsymbol{F}, \boldsymbol{H}, \boldsymbol{\Sigma}_{\boldsymbol{F}}$ and $\boldsymbol{\Sigma}_{\boldsymbol{H}}$ and piecewise constant interpolation of the Jacobian $J$ and its associated stress conjugate $\Sigma_{J}$. With these functional spaces, the three mixed formulations $\mathbf{M} 7 \mathbf{F}, \mathbf{M C F}$ and $\mathbf{M} 5 \mathbf{F}$ will render identical results.

For the incompressible case, a two field $\{\boldsymbol{x}, p\}$ mixed formulation is employed, as described in Section 4.4. In this case, two interpolations will be compared. First, a P1-P1 linear continuous interpolation for both displacement and pressure fields (e.g. with the help of stabilisation) and, second, a P2-P0 continuous quadratic interpolation for the displacement field and piecewise constant interpolation for the pressure field (e.g. without stabilisation).

From the post-processing standpoint, the numerical results in terms of stresses will be reported for the Cauchy stress components $\boldsymbol{\sigma}$ and the hydrostatic pressure $p$ (e.g. $p=\frac{1}{3} \boldsymbol{\sigma}: \boldsymbol{I}$ ). It is important to emphasise that their evaluation is carried out via equation (82). 


\subsection{Patch test}

The first numerical example includes a standard three dimensional patch test in order to assess the correctness of the computational implementation. This problem was already presented in [24]. Two different polyconvex compressible constitutive models are considered. The first polyconvex model is a standard Mooney-Rivlin model, based on equation (33), as follows:

$$
\begin{aligned}
W_{s}(\boldsymbol{F}, \boldsymbol{H}, J) & =\alpha_{s}(\boldsymbol{F}: \boldsymbol{F})+\beta_{s}(\boldsymbol{H}: \boldsymbol{H})+f_{s}(J) \\
f_{s}(J) & =-4 \beta_{s} \ln J-2 \alpha_{s} \ln J+\frac{\lambda_{s}}{2 \varepsilon_{s}^{2}}\left(J^{\varepsilon_{s}}-J^{-\varepsilon_{s}}\right)
\end{aligned}
$$

where $\alpha_{s}, \beta_{s}, \lambda_{s}$ and $\varepsilon_{s}$ are user defined material parameters given by

$$
\alpha_{s}=126 k P a, \quad \beta_{s}=252 k P a, \quad \lambda_{s}=81512 k P a, \quad \varepsilon_{s}=20 .
$$

A second polyconvex constitutive model, based on that presented in [24], is defined as follows,

$$
\begin{aligned}
W_{q}(\boldsymbol{F}, \boldsymbol{H}, J) & =\alpha_{q}(\boldsymbol{F}: \boldsymbol{F})^{2}+\beta_{q}(\boldsymbol{H}: \boldsymbol{H})^{2}+f_{q}(J) \\
f_{q}(J) & =-24 \beta_{q} \ln J-12 \alpha_{q} \ln J+\frac{\lambda_{q}}{2 \varepsilon_{q}^{2}}\left(J^{\varepsilon_{q}}-J^{-\varepsilon_{q}}\right)
\end{aligned}
$$

where $\alpha_{q}, \beta_{q}, \lambda_{q}$ and $\varepsilon_{q}$ are user defined material parameters. Note that for this second polyconvex model $W_{q}, \boldsymbol{F}$ and $\boldsymbol{H}$ can be expressed in terms of their respective conjugate stresses $\boldsymbol{\Sigma}_{\boldsymbol{F}}$ and $\boldsymbol{\Sigma}_{\boldsymbol{H}}$, respectively, as,

$$
\begin{aligned}
\boldsymbol{F} & =\frac{1}{4 \alpha_{q}}\left(\boldsymbol{\Sigma}_{\boldsymbol{F}}: \boldsymbol{\Sigma}_{\boldsymbol{F}}\right)^{-1 / 3} \boldsymbol{\Sigma}_{\boldsymbol{F}} \\
\boldsymbol{H} & =\frac{1}{4 \beta_{q}}\left(\boldsymbol{\Sigma}_{\boldsymbol{H}}: \boldsymbol{\Sigma}_{\boldsymbol{H}}\right)^{-1 / 3} \boldsymbol{\Sigma}_{\boldsymbol{H}} .
\end{aligned}
$$

Above equations (166) enable the explicit computation of the strain variables in terms of their stress conjugates without having to resort to a numerical solution via a Newton-Raphson nonlinear solver. This will be of interest when using the complementary energy mixed formulation MCF. On the contrary, the Jacobian $J$ can only be obtained in terms of its conjugate stress $\Sigma_{J}$ after solving numerically above equations (163b) and (165b).

The material parameters used for this constitutive model are taken as:

$$
\alpha_{q}=21 k P a, \quad \beta_{q}=42 k P a, \quad \lambda_{q}=8000 k P a, \quad \varepsilon_{q}=20 .
$$


For both constitutive models $W_{s}$ and $W_{q}$, the linear elasticity constitutive operator in the reference configuration renders the same material parameters, namely shear modulus $\mu=756 k \mathrm{~Pa}$ and Poisson's ratio $\nu=0.49546$.

A homogeneous deformation mapping is defined through a stretch $\Delta L / L=$ 0.5 in the $O X$ direction, applied to a cubic shape domain of side $L$, as depicted in Figure 2. To achieve this deformation, non-zero normal Dirichlet boundary conditions are applied on the boundary faces perpendicular to the $O X$ axis and zero normal Dirichlet boundary conditions are defined on two adjacent faces perpendicular to the $O Y$ and $O Z$ axes. Zero Neumann boundary conditions are defined everywhere else.

The domain is discretised using two different meshes of $(2 \times 2 \times 2) \times 6$ tetrahedral elements. First, a structured mesh is shown in Figure 2(a) and, second, a distorted mesh is shown in Figure 2(b) (as presented in reference [24]) where the interior node is displaced randomly. The objective of this example is to demonstrate that the same solution is obtained for both meshes.

As expected, hence passing the patch test, for the three mixed formulations defined above, namely $\mathbf{M} 7 \mathbf{F}, \mathbf{M C F}$ and $\mathbf{M} 5 \mathbf{F}$, the results are identical for both meshes (the only numerical difference due to machine accuracy). A resulting Cauchy stress component of $\sigma_{x x}=929.9 \mathrm{kPa}$ is obtained for the model $W_{s}$ and a value of $\sigma_{x x}=1,087.4 k P a$ is obtained for the model $W_{q}$, which is identical to that reported in reference [24]. The homogeneous deformation gradient tensors for both constitutive models, namely $\boldsymbol{F}_{W_{s}}$ and $\boldsymbol{F}_{W_{q}}$, are

$$
\boldsymbol{F}_{W_{s}}=\left[\begin{array}{ccc}
1.5 & 0 & 0 \\
0 & 0.8161 & 0 \\
0 & 0 & 0.8161
\end{array}\right], \quad \boldsymbol{F}_{W_{q}}=\left[\begin{array}{ccc}
1.5 & 0 & 0 \\
0 & 0.8170 & 0 \\
0 & 0 & 0.8170
\end{array}\right]
$$

For completeness, the quadratic convergence of the Newton-Raphson algorithm is displayed in Figure 3. 


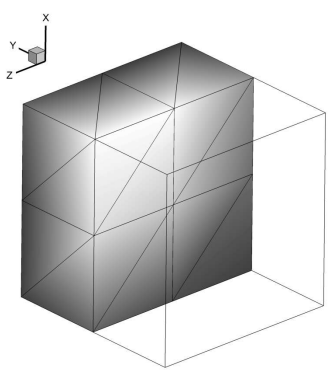

(a)

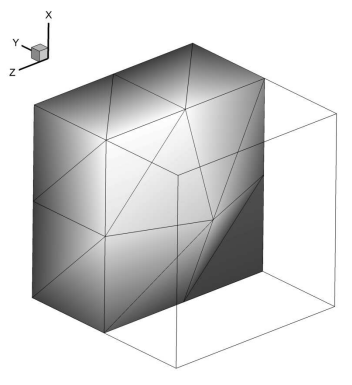

(b)

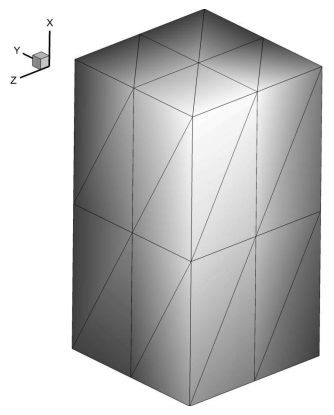

(c)

Figure 2: Three dimensional patch test. (a) View of half undistorted mesh in the reference configuration. (b) View of half distorted mesh in the reference configuration. (c) Example of deformed geometry after stretching of $\Delta L / L=0.5$ in the $O X$ direction for a MooneyRivlin model $W_{s}$ defined in (163) with parameters defined in (164).

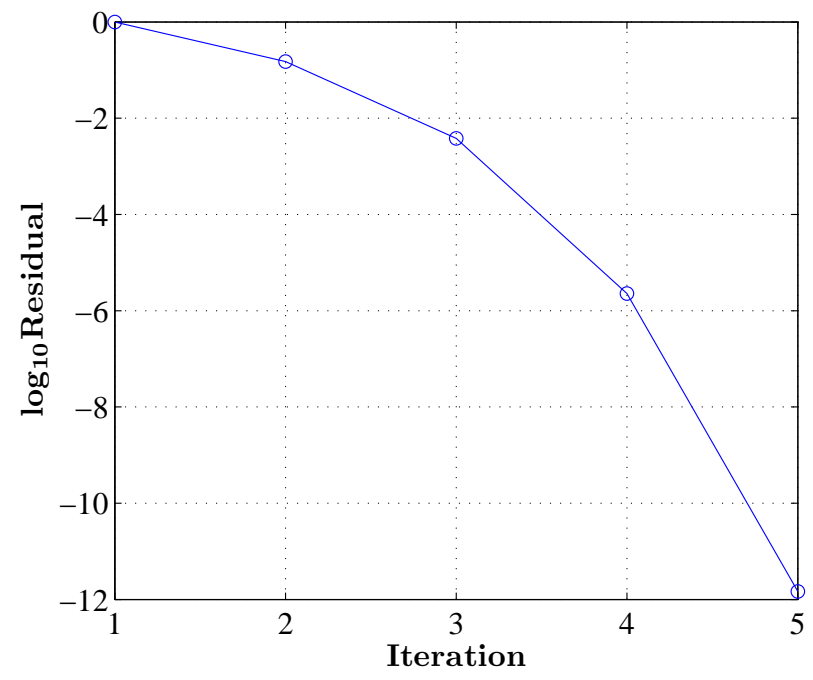

Figure 3: Three dimensional patch test. Quadratic convergence of the Newton-Raphson linearisation procedure. 


\subsection{Cook type cantilever problem.}

In this section, we analyse the same problem presented in [24] where a Cook type cantilever problem of thickness $t=10 \mathrm{~m}$ is analysed. The geometry of the problem is shown in Figure 4(a) where, as it can be observed, the cantilever is clamped on its left end and subjected to an upwards parabolic shear force distribution applied on its right end of maximum value $\tau_{\max }=$ $16 \mathrm{kPa}$. Note that this force is not considered to be a follower-load during the deformation process.

The problem is analysed with two polyconvex compressible constitutive models. The first polyconvex model is given by a Mooney-Rivlin material defined as,

$$
\begin{array}{r}
W_{p}(\boldsymbol{F}, \boldsymbol{H}, J)=\alpha_{p}(\boldsymbol{F}: \boldsymbol{F})+\beta_{p}(\boldsymbol{H}: \boldsymbol{H})+f_{p}(J) \\
f_{p}(J)=-4 \beta_{p} \ln J-2 \alpha_{p} \ln J+\frac{\lambda_{p}}{2}(J-1)^{2}
\end{array}
$$

where the material parameters are chosen as

$$
\alpha_{p}=126 k P a, \quad \beta_{p}=252 k P a, \quad \lambda_{p}=81512 k P a .
$$

The second polyconvex model, $W_{q}$, is identical to that presented in (165) with material parameters those in (167). This renders for both $W_{p}$ and $W_{q}$ models an identical linear elasticity operator in the reference state defined by a shear modulus $\mu=756 \mathrm{kPa}$ and a Poisson's ratio $\nu=0.49546$. The nearly incompressible nature of the material will emphasise the differences between the $\mathbf{D F}$ formulation and the alternative mixed formulations, particularly in terms of stresses.

Notice that in the case of the polyconvex model defined by $W_{p}$, the stress conjugate $\Sigma_{J}$ to the Jacobian $J$ is obtained as,

$$
\Sigma_{J}=-\frac{2\left(\alpha_{p}+2 \beta_{p}\right)}{J}+\lambda_{p}(J-1) .
$$

Notice also that this simple expression enables, without the need to employ a Newton-Raphson procedure, to express directly the Jacobian $J$ in 


\begin{tabular}{|c|c|c|c|c|}
\hline Mesh & Elems. & Dofs. $\boldsymbol{x}$ & Dofs. $\boldsymbol{F}, \boldsymbol{H}, \boldsymbol{\Sigma}_{\boldsymbol{F}}, \boldsymbol{\Sigma}_{\boldsymbol{H}}$ & Dofs. $J, \Sigma_{J}$ \\
\hline Coarse & 1,470 & $2,475 \times 3$ & $1,470 \times 4 \times 9$ & $1,470 \times 1$ \\
Medium & 3,600 & $5,730 \times 3$ & $3,600 \times 4 \times 9$ & $3,600 \times 1$ \\
Fine & 5,880 & $9,251 \times 3$ & $5,880 \times 4 \times 9$ & $5,880 \times 1$ \\
\hline
\end{tabular}

Table 1: Cook type cantilever problem. Mesh discretisation details. Column 2: number of tetrahedral elements (Elems.). Column 3: number of degrees of freedom (Dofs.) associated to the spatial coordinates $\boldsymbol{x}$. Column 4: number of degrees of freedom (Dofs.) associated to the strain/stress fields $\boldsymbol{F}, \boldsymbol{H}, \boldsymbol{\Sigma}_{\boldsymbol{F}}, \boldsymbol{\Sigma}_{\boldsymbol{H}}$. Column 5: number of degrees of freedom (Dofs.) associated to the strain/stress fields $J, \Sigma_{J}$.

terms of its conjugate stress $\Sigma_{J}$ as follows,

$$
\begin{array}{r}
J=\frac{\left(\lambda_{s}+\Sigma_{J}\right)+\sqrt{\left(\lambda_{s}+\Sigma_{J}\right)^{2}+8 \lambda_{s}\left(\alpha_{s}+2 \beta_{s}\right)}}{2 \lambda_{s}}, \text { if } \lambda_{s}>0 \\
J=-\frac{2\left(\alpha_{s}+2 \beta_{s}\right)}{\Sigma_{J}}, \text { if } \lambda_{s}=0 .
\end{array}
$$

These expressions are particularly useful when using the complementary energy formulation MCF. Three discretisations are considered, namely coarse, medium and fine, comprised of $(7 \times 7 \times 5) \times 6,(10 \times 10 \times 6) \times 6$ and $(14 \times 14 \times 5) \times 6$ tetrahedral elements, respectively. The fine mesh is displayed, as an example, in all of the numerical results presented in Figures 4(c)-(d) and thereafter. For completeness, Table 1 displays the discretisation details for each of the meshes employed. Notice how the number of degrees of freedom associated to the strain/stress variables is proportional to the number of tetrahedral elements of the mesh. As presented in previous sections, these degrees of freedom are condensed out at an element level.

For the three discretisations and the two constitutive models above described, the four implementations DF, M7F, MCF and M5F where analysed. For instance, Figures 4(c) and 4(d) display the displacement field $u_{y}$ and the stress field $\sigma_{x x}$ for the fine discretisation and the constitutive model $W_{q}$ by using the various mixed formulations (i.e. the three implementations render identical results).

Figures 5 to 7 display the contour plot of different stress magnitudes $\left(\sigma_{x x}\right.$, $\sigma_{y y}$ and pressure) using the constitutive model $W_{p}$ and the fine discretisa- 
tion. Results are presented comparing the mixed formulations, which render identical results (see Figures 5(a), 6(a) and 7(a)) versus the DF formulation (see Figures 5(b), 6(b) and 7(b)).

A more detailed comparison is established in Tables 2 and 3, where results obtained with the four formulations, namely the three mixed formulations and the DF formulation are displayed. Results are presented for both stresses and displacements sampled at points $A, B$ and $C$, as depicted in Figure 4(b). As can be noticed, the $\mathbf{D F}$ implementation underestimates the displacements obtained with the alternative mixed formulations. It can be seen how the DF implementation converges from below whereas the alternative mixed formulations converge from above. In addition, the results obtained with the constitutive model $W_{q}$ match very well those presented in reference [24].

As expected (e.g nearly incompressible material), regarding the stresses, the differences are more significant, specially for the $W_{q}$ constitutive model. The higher nonlinearity of the $W_{q}$ model with respect to the $W_{p}$ model highlights the differences between both formulations. Whereas the results for the DF formulation do not seem to converge with clear pressure oscillations, the results for the alternative mixed formulations show a very defined convergence pattern. The results obtained with the constitutive model $W_{q}$ match very well those presented in reference [24].

Finally, it is important to mention that the equivalence between the $\mathbf{M C F}$ formulation and the other mixed formulations can be badly affected with the choice of the parameter $\varepsilon_{q}$ used in equation $(165)\left(\varepsilon_{s}\right.$ in equation (163)). The higher the coefficient $\varepsilon_{q}\left(\varepsilon_{s}\right)$ the higher the error tolerance must be when solving numerically equation (165b) (or equation (163b)) via the NewtonRaphson nonlinear solver. Otherwise, small variations in the Jacobian $J$ can introduce significant changes in the conjugate stress $\Sigma_{J}$ and make the MCF formulation to yield different results to those of the $\mathbf{M 7 F}$ and $\mathbf{M 5 F}$ formulations. 


\begin{tabular}{|r|rrr|rrr|} 
& \multicolumn{3}{c}{$W_{p}$ model } & \multicolumn{3}{c}{$W_{q}$ model } \\
\cline { 2 - 7 } & Fine & Medium & Coarse & Fine & Medium & Coarse \\
\hline$\sigma_{x x}^{A}$ & -103.12 & -105.31 & -106.64 & -114.36 & -117.63 & -116.46 \\
$\sigma_{x x}^{B}$ & 120.35 & 121.98 & 118.17 & 171.37 & 172.83 & 166.76 \\
$\sigma_{y y}^{A}$ & -113.89 & -116.18 & -117.27 & -147.38 & -150.96 & -148.07 \\
$\sigma_{y y}^{B}$ & 196.49 & 197.99 & 194.78 & 366.43 & 365.96 & 364.85 \\
$u_{x}^{C}$ & -20.76 & -20.78 & -20.81 & -20.32 & -20.30 & -20.25 \\
$u_{y}^{C}$ & 18.93 & 18.95 & 18.98 & 18.66 & 18.66 & 18.66 \\
$u_{z}^{C}$ & 0.010 & 0.016 & 0.036 & -0.001 & -0.0005 & 0.007 \\
\hline
\end{tabular}

Table 2: Cook type cantilever problem. Stress components $(k P a)$ and displacements $(m)$ at points $A, B$ and $C$ as displayed in Figure 4(b). Results are obtained using the mixed formulations. Coarse, medium and fine discretisations of $(7 \times 7 \times 5) \times 6,(10 \times 10 \times 6) \times 6$ and $(14 \times 14 \times 5) \times 6$ tetrahedral elements, respectively. $W_{p}$ model (columns 2 to 4$)$ defined in (169) with parameters given in (170). $W_{q}$ model (columns 5 to 7 ) defined in (165) with material parameters in (167).

\begin{tabular}{|r|rrr|rrr|} 
& \multicolumn{3}{c}{$W_{p}$ model } & \multicolumn{3}{c}{$W_{q}$ model } \\
\cline { 2 - 7 } & Fine & Medium & Coarse & Fine & Medium & Coarse \\
\hline$\sigma_{x x}^{A}$ & -94.40 & -75.28 & -189.83 & -98.39 & -79.41 & -192.31 \\
$\sigma_{x x}^{B}$ & 232.99 & 305.44 & 88.03 & 238.37 & 310.57 & 94.58 \\
$\sigma_{y y}^{A}$ & -134.17 & -114.81 & -223.26 & -132.36 & -115.06 & -221.94 \\
$\sigma_{y y}^{B}$ & 419.31 & 485.52 & 279.44 & 426.22 & 492.28 & 286.87 \\
$u_{x}^{C}$ & -20.55 & -20.43 & -20.21 & -20.20 & -20.00 & -19.86 \\
$u_{y}^{C}$ & 18.83 & 18.80 & 18.74 & 18.60 & 18.57 & 18.51 \\
$u_{z}^{C}$ & -0.060 & -0.086 & -0.143 & -0.061 & -0.087 & -0.143 \\
\hline
\end{tabular}

Table 3: Cook type cantilever problem. Stress components $(\mathrm{kPa})$ and displacements $(m)$ at points $A, B$ and $C$ as displayed in Figure 4(b). Results obtained using the DF formulation. Coarse, medium and fine discretisations of $(7 \times 7 \times 5) \times 6,(10 \times 10 \times 6) \times 6$ and $(14 \times 14 \times 5) \times 6$ tetrahedral elements, respectively. $W_{p}$ model (columns 2 to 4$)$ defined in (169) with parameters given in (170). $W_{q}$ model (columns 5 to 7 ) defined in (165) with material parameters in (167). 


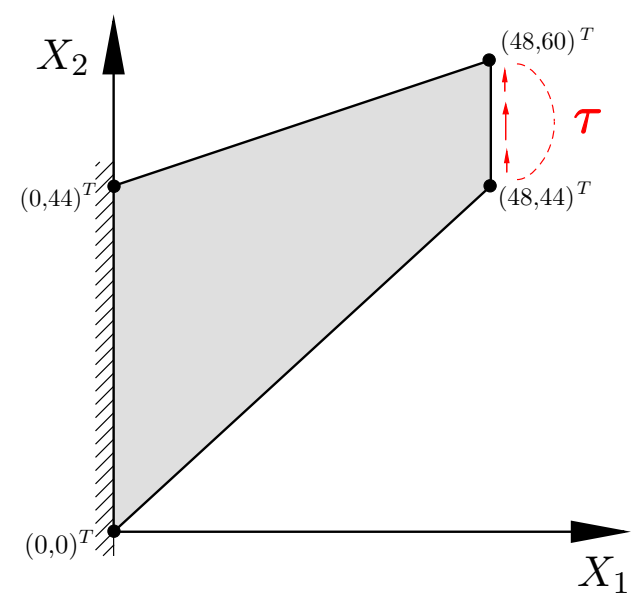

(a)

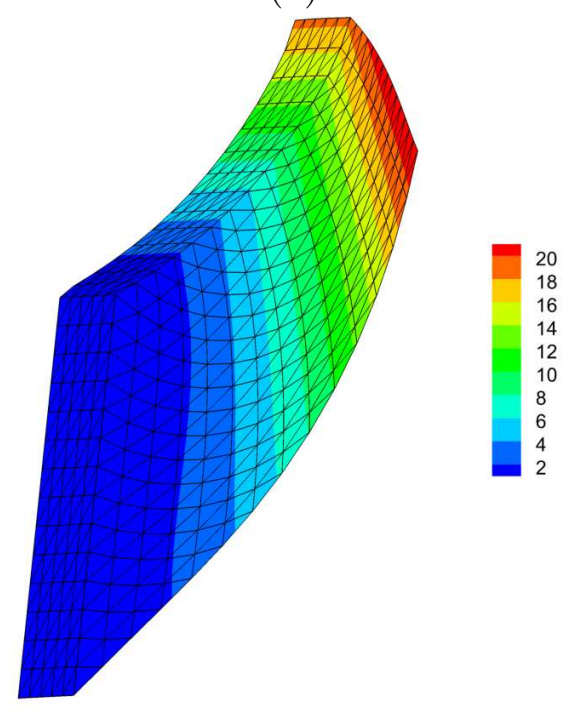

(c)

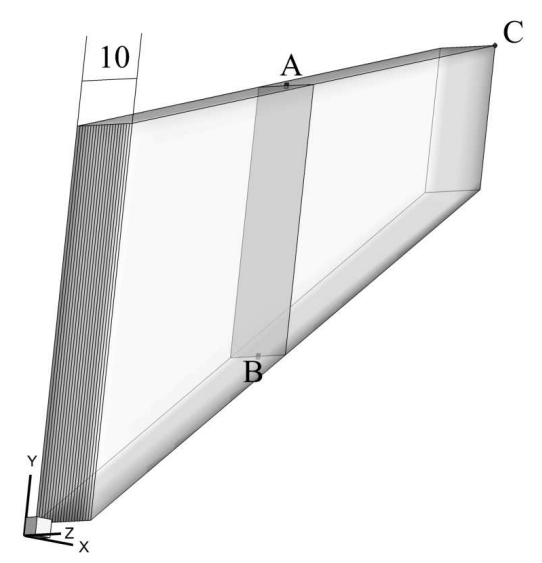

(b)

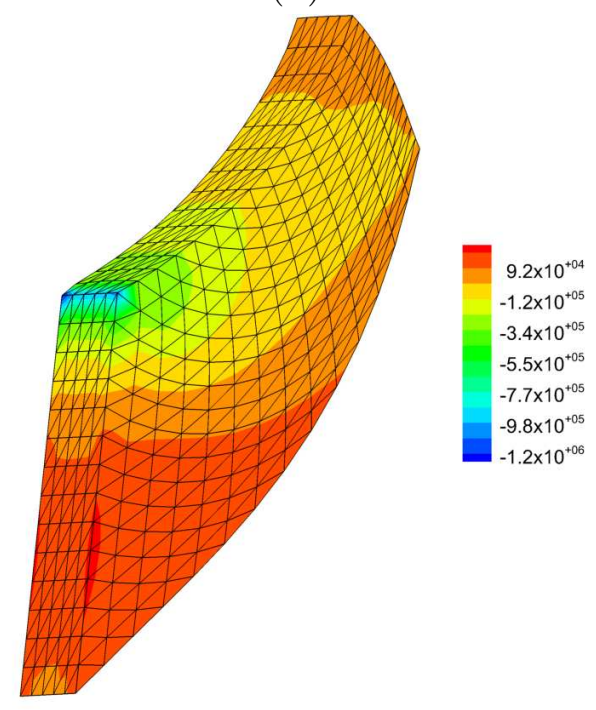

(d)

Figure 4: Cook type cantilever problem. (a) Geometry of the problem and boundary conditions, where a parabolic upwards shear stress distribution with maximum value $\tau_{\max }=300 \mathrm{kPa}$ is applied on its right end. Axes $O X_{1}$ and $O X_{2}$ coincide with $o x$ and $o y$ in (b), respectively. (b) Representation of the finest discretisation employed with $(14 \times 14 \times 5) \times 6$ tetrahedral elements. (c) Contour plot of displacement field $u_{y}(m)$ for the polyconvex model $W_{q}$ defined in (165) with material parameters in (167). (d) Contour plot of stress field $\sigma_{x x}(\mathrm{~Pa})$ for the polyconvex model $W_{q}$ defined in (165) with material parameters in (167). 


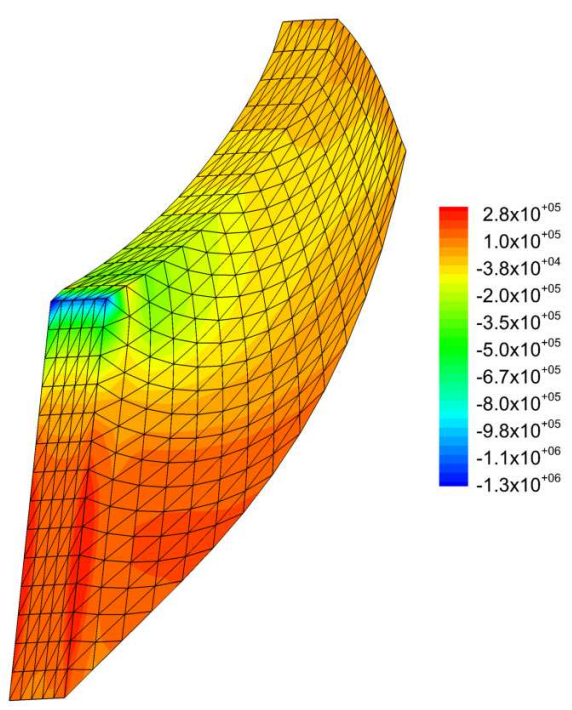

(a)

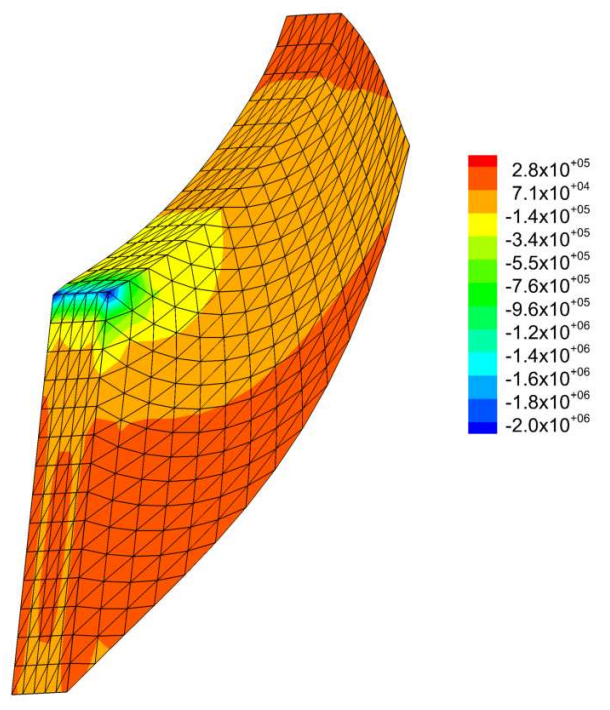

(b)

Figure 5: Cook type cantilever problem. Contour plot of the distribution of stress coefficient $\sigma_{x x}(\mathrm{~Pa})$ for (a) mixed formulations and (b) DF formulation. Mooney-Rivlin model $W_{p}$ defined in (169) with material parameters given in (170). Discretisation of $(14 \times 14 \times 5) \times 6$ tetrahedral elements. 


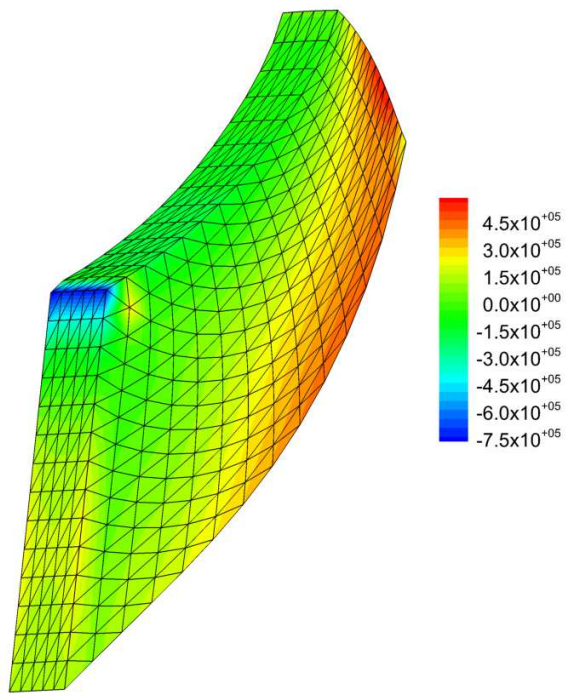

(a)

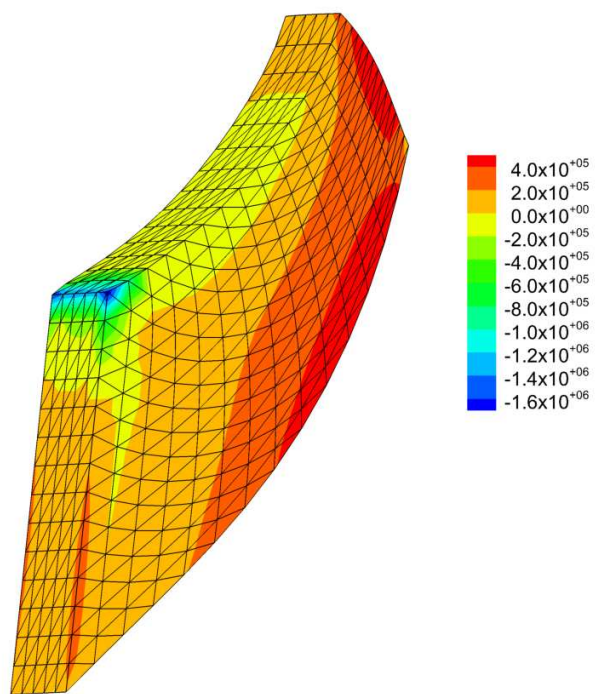

(b)

Figure 6: Cook type cantilever problem. Contour plot of the distribution of stress coefficient $\sigma_{y y}(\mathrm{~Pa})$ for (a) mixed formulations and (b) DF formulation. Mooney-Rivlin model $W_{p}$ defined in (169) with material parameters given in (170). Discretisation of $(14 \times 14 \times 5) \times 6$ tetrahedral elements. 


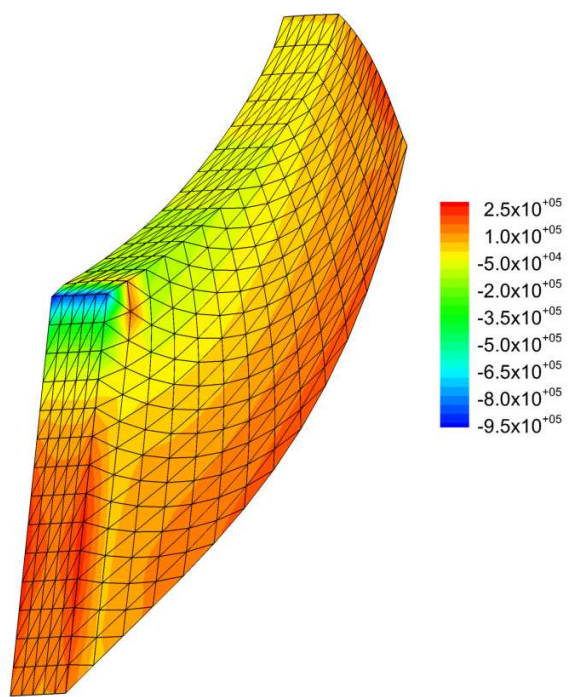

(a)

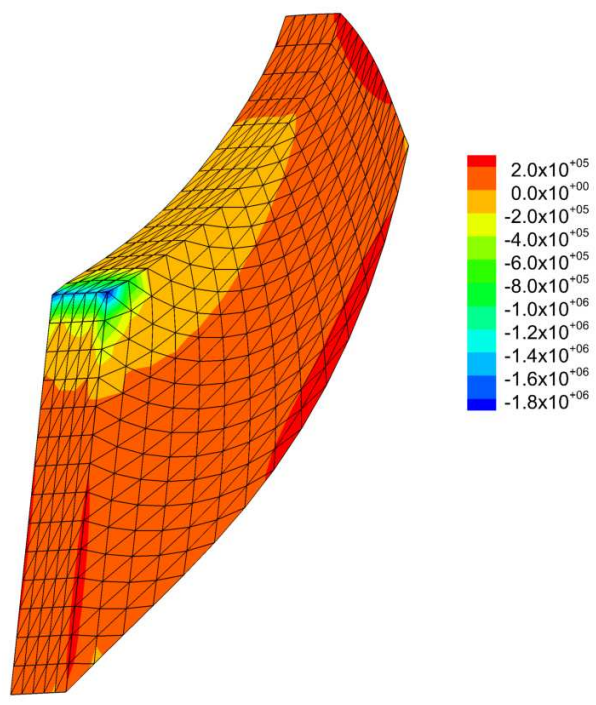

(b)

Figure 7: Cook type cantilever problem. Contour plot of the distribution of the hydrostatic pressure $p(\mathrm{~Pa})$ for (a) mixed formulations and (b) DF formulation. Mooney-Rivlin model $W_{p}$ defined in (169) with material parameters given in (170). Discretisation of $(14 \times 14 \times 5) \times 6$ tetrahedral elements. 


\subsection{Compressible short column subjected to transverse shear force.}

In this example, a short column of squared cross sectional area is analysed when subjected to large deformations, as depicted in Figure 8(a). The height of the column is $h=5 m$ and the size of the square defining the cross section is $a=1 \mathrm{~m}$. The column is clamped at its bottom end and it is subjected at the top end to a shear stress distribution parabolic in the $O Y$ direction and constant in the $O X$ direction, of maximum value $\tau_{\max }=76 \mathrm{kPa}$. Note that this force is not considered to be a follower-load during the deformation process.

The constitutive model is a compressible Mooney-Rivlin type material defined by an energy function $W_{c}$ as

$$
\begin{aligned}
W_{c}(\boldsymbol{F}, \boldsymbol{H}, J) & =\alpha_{c}(\boldsymbol{F}: \boldsymbol{F})+\beta_{c}(\boldsymbol{H}: \boldsymbol{H})+f_{c}(J) \\
f_{c}(J) & =-4 \beta_{c} \ln J-2 \alpha_{c} \ln J
\end{aligned}
$$

where the material parameters are chosen as

$$
\alpha_{c}=47.25 \mathrm{kPa}, \quad \beta_{c}=141.75 \mathrm{kPa} .
$$

The selection of these material parameters yield a linear elasticity constitutive operator in the origin defined by a shear modulus $\mu=378 \mathrm{kPa}$ and a Poisson's ratio $\nu=0.3$.

The objective of this example is to demonstrate the $p$-order of accuracy of the different mixed formulations, as a function of the chosen finite element approximation spaces. For this purpose, the column is initially discretised with $(2 \times 2 \times 10) \times 6$ tetrahedral elements (see Figure $8(\mathrm{~b}))$ and, subsequently, $h$-refinement is carried out generating a total of four discretisations. As a closed form solution is not available for this problem, the finest mesh is used to generate numerically the so-called "benchmark" solution (for each mixed formulation) for comparison purposes. The error between the benchmark

solution and the other discretisations is measured in the $L^{2}$ norm for all the unknown variables.

Let us define for a tensor (e.g. scalar, vector or second order) field, the $L^{2}$ norm as

$$
\|\boldsymbol{\zeta}\|_{L^{2}}=\left[\int_{V}(\boldsymbol{\zeta}: \boldsymbol{\zeta}) d V\right]^{1 / 2}
$$

associated with the magnitude of the tensor field $\boldsymbol{\zeta}$. In our case, $\boldsymbol{\zeta}$ can be any of the kinematic or kinetic unknowns, namely $\boldsymbol{x}, \boldsymbol{F}, \boldsymbol{H}, J, \boldsymbol{\Sigma}_{\boldsymbol{F}}, \boldsymbol{\Sigma}_{\boldsymbol{H}}$ and $\Sigma_{J}$. 
This enables the definition of the following error norm $\left\|\boldsymbol{\zeta}_{i}-\boldsymbol{\zeta}_{b}\right\|_{L^{2}} /\left\|\boldsymbol{\zeta}_{b}\right\|_{L^{2}}$, where $\boldsymbol{\zeta}_{b}$ stands for the benchmark solution and $\boldsymbol{\zeta}_{i}$ the solution of the $i$-th mesh, with $i=1, \ldots,(b-1)$. This can then be used to assess the convergence of the algorithm under $h$-refinement.

Figure 9 displays the contour plot distribution of the stress $\sigma_{x x}$ for different stages of the deformation. The results presented are identical for all of the mixed formulations. As can be observed, the stress distribution is smooth (absence of pressure modes) and the deformation does not show any locking in its deformation pattern.

Figure 10 shows the order of accuracy of the different unknown variables for the mixed formulations (all yielding identical convergence pattern). Figure 10(a) displays the convergence of the kinematic variables $\boldsymbol{x}, \boldsymbol{F}, \boldsymbol{H}$ and $J$ whereas Figure 10(b) displays the convergence of the kinetic variables $\boldsymbol{\Sigma}_{\boldsymbol{F}}, \boldsymbol{\Sigma}_{\boldsymbol{H}}$ and $\Sigma_{J}$. As expected, the convergence observed is $p+1$ in all the variables.

For completeness, Figure 11 has been included to demonstrate the quadratic convergence of the Newton-Raphson linearisation procedure. 


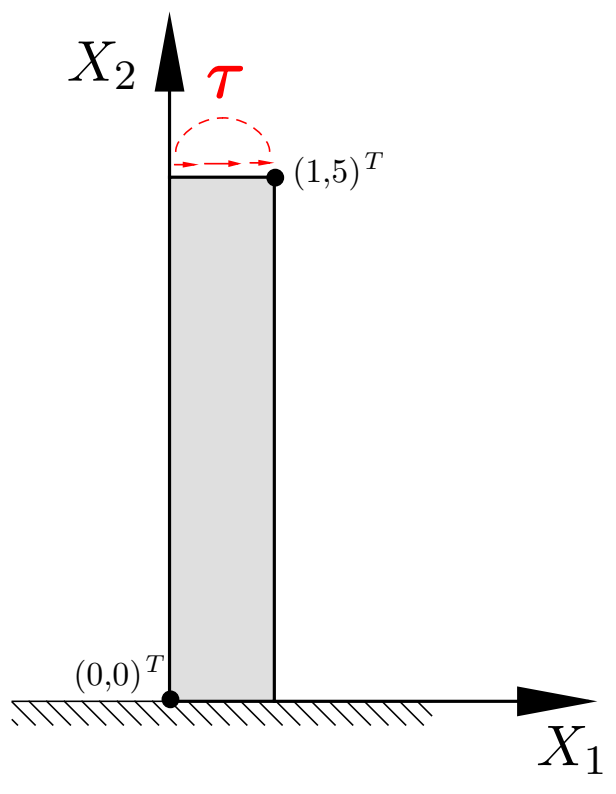

(a)

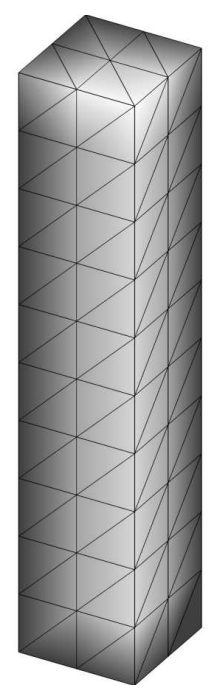

(b)

Figure 8: Compressible short column of height $h=5 \mathrm{~m}$ and squared cross section defined by its size $a=1 \mathrm{~m}$. (a) Boundary conditions: clamped bottom end and parabolic stress distribution at the top end. Axes $O X_{1}$ and $O X_{2}$ coincide with $o y$ and $o z$ in (b), respectively. (b) Example of finite element discretisation: coarsest mesh with $(2 \times 2 \times 10) \times 6$ tetrahedral elements. 


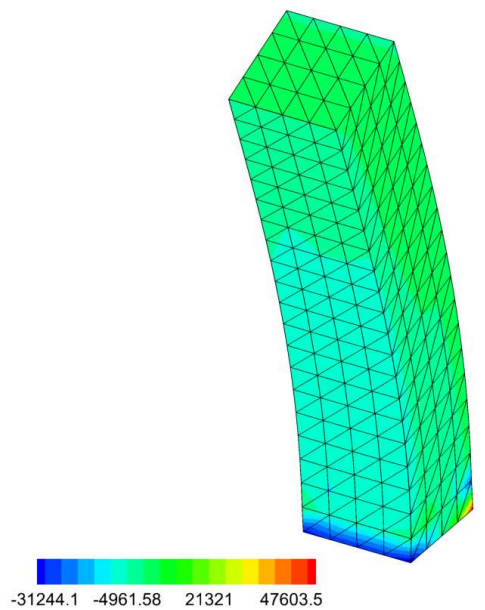

(a)

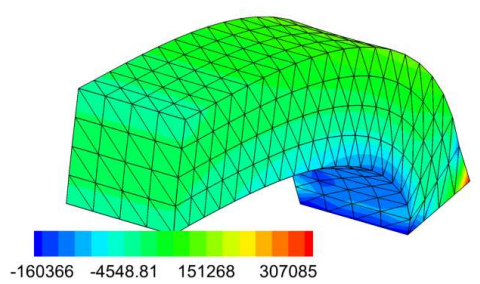

(c)

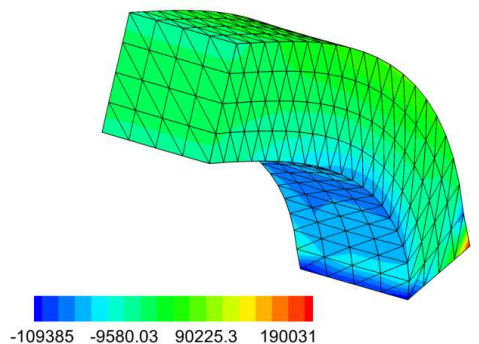

(b)

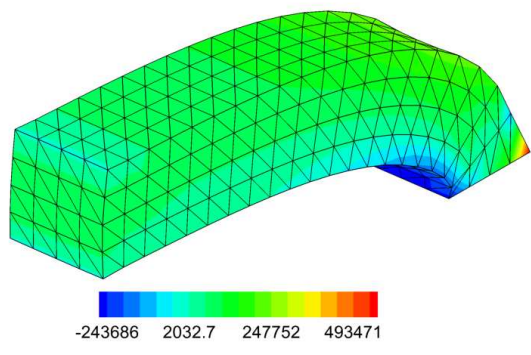

(d)

Figure 9: Contour plot of the stress $\sigma_{x x}(\mathrm{~Pa})$ distribution for the compressible short column example using a mixed formulation. Results after application of an incremental loading of (a) $5 \%$ (b) $25 \%$ (c) $50 \%$ and (d) $100 \%$ of the total external shear stress $\tau=\tau_{\max }$. MooneyRivlin model $W_{p}$ defined in (169) with material parameters given in (174). Results shown for a discretisation of $(4 \times 4 \times 20) \times 6$ tetrahedral elements $(3,321 \times 3$ degrees of freedom associated to the spatial coordinates $\boldsymbol{x}$ ). 


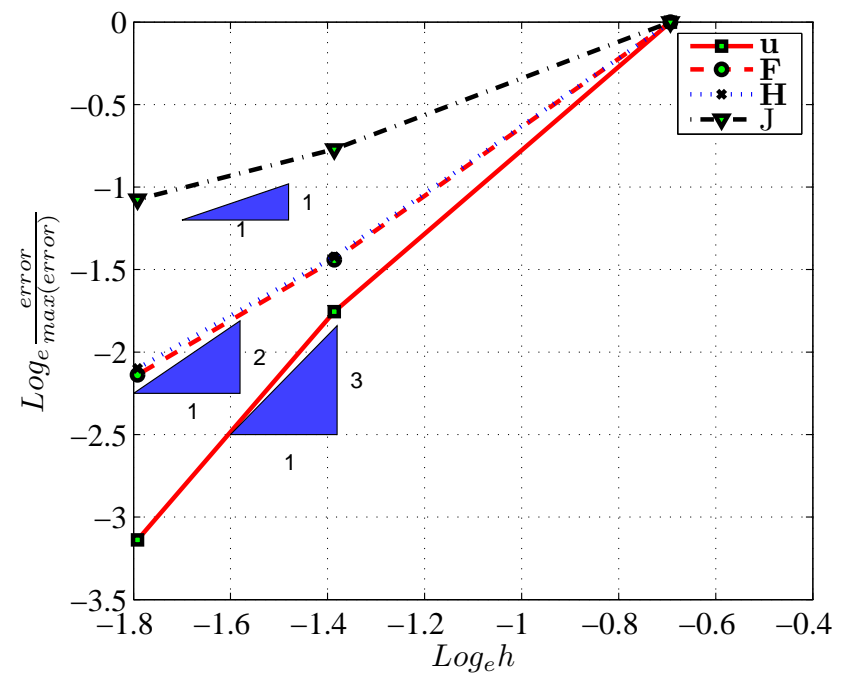

(a)

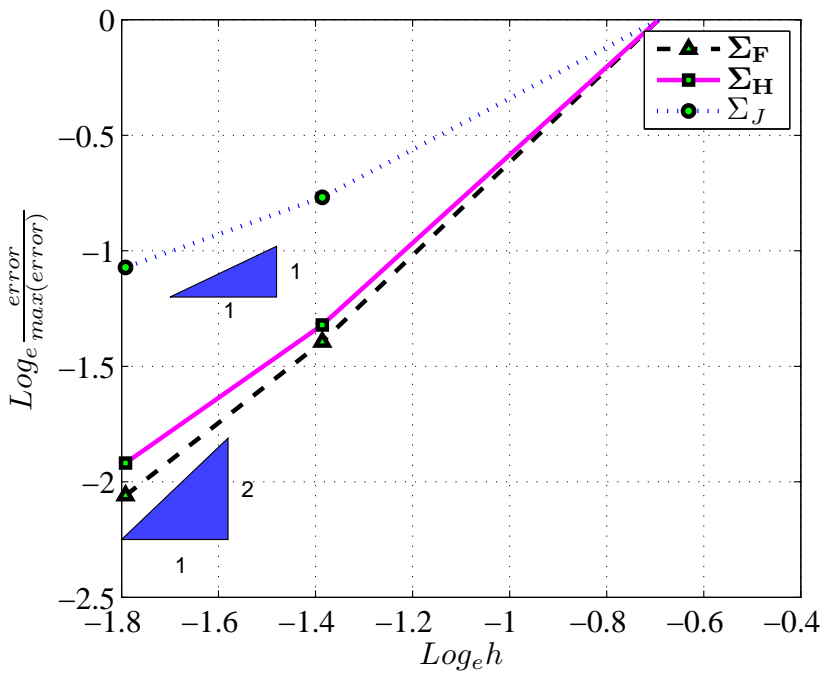

(b)

Figure 10: Compressible short column example: order of accuracy of different strain and stress magnitudes for the mixed formulations. (a) Order of accuracy of the kinematic variables $\boldsymbol{x}, \boldsymbol{F}, \boldsymbol{H}$ and $J$. (b) Order of accuracy of the kinetic variables $\boldsymbol{\Sigma}_{\boldsymbol{F}}, \boldsymbol{\Sigma}_{\boldsymbol{H}}$ and $\Sigma_{J}$. Mooney-Rivlin model $W_{p}$ defined in (169) with material parameters given in (174). 


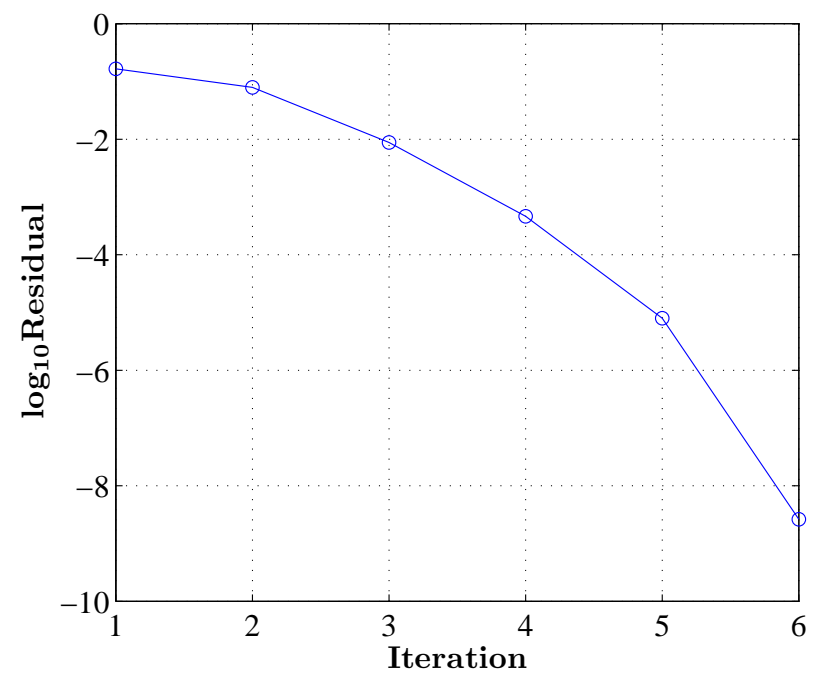

Figure 11: Compressible short column example: quadratic convergence of the NewtonRaphson linearisation procedure. Mooney-Rivlin model $W_{p}$ defined in (169) with material parameters given in (174). 


\subsection{Incompressible long column subjected to transverse shear force.}

A very similar example to that presented in the previous section is analysed. In this case, a longer column of height $h=10 \mathrm{~m}$ of squared cross sectional area is analysed when subjected to large deformations. As in the previous section, the size of the square defining the cross section remains $a=1 \mathrm{~m}$. The column is clamped at its bottom end and subjected to a parabolic (only in the $O Y$ direction) shear stress distribution at the top end of maximum value $\tau_{\max }=14.4 \mathrm{kPa}$. Note that this force is not considered to be a follower-load during the deformation process.

The constitutive model is taken as incompressible Mooney-Rivlin defined via a polyconvex isochoric energy function $\hat{W}_{i}$ defined as

$$
\hat{W}_{i}(\boldsymbol{F}, \boldsymbol{H}, J)=\nu_{i} J^{-2 / 3}(\boldsymbol{F}: \boldsymbol{F})+\gamma_{i} J^{-2}(\boldsymbol{H}: \boldsymbol{H})^{3 / 2}
$$

where the material parameters are chosen as

$$
\nu_{i}=189 k P a, \quad \gamma_{i}=72.75 k P a .
$$

The selection of these material parameters yield a linear elasticity constitutive operator in the origin defined by shear modulus $\mu=756 \mathrm{kPa}$ and Poisson's ratio $\nu=0.5$.

The objective of this example is to demonstrate the behaviour of the stabilisation technique as presented in Section 5.3. For this purpose, two interpolation techniques will be considered: first, a P1-P1 linear continuous interpolation for both displacement and pressure fields (e.g. with the help of stabilisation) and, second, a P2-P0 continuous quadratic interpolation for the displacement field and piecewise constant interpolation for the pressure field (e.g. without stabilisation).

For the P1-P1 interpolation, an unstructured mesh of 17,575 tetrahedral elements is used $(3,859 \times 3$ degrees of freedom associated to the spatial coordinates $\boldsymbol{x}$ ) (refer to Figures 12(a)-(c)) and for the P2-P0 interpolation, an unstructured mesh of 3,962 tetrahedral elements is used (6,675 $\times 3$ degrees of freedom associated to the spatial coordinates $\boldsymbol{x}$ ) (refer to Figures 12(d)). Notice that a very fine P2-P0 discretisation has been chosen in order to adequately benchmark the solution obtained with the alternative stabilised P1-P1 formulation.

Figures 12 and 13 display the contour plot distribution of the hydrostatic pressure and the displacement field $u_{x}$, respectively. Within these figures, the solution by using the non-stabilised P2-P0 implementation is displayed in the 
sub-figures (d) whereas sub-figures (a) to (c) present the results for the stabilised P1-P1 implementation, with stabilisation parameter $\alpha=0.1,0.2,0.5$, respectively. In all of the $\mathrm{P} 1-\mathrm{P} 1$ analyses, stabilisation coefficient $\tau_{p}=0$ was employed.

The results show an excellent agreement between the non-stabilised P2-P0 formulation (see Figures 12(d) and 13(d)) and the stabilised P1-P1 formulation when using $\alpha=0.2$ (see Figures 12(b) and 13(b)). Larger values of the stabilisation parameter $\alpha$ lead to stiffer solutions (i.e. volumetric and shear locking) and viceversa. It is also clear that for the stabilised P1-P1 implementation, smooth distributions of stresses are obtained, removing possible spurious pressure oscillations. 


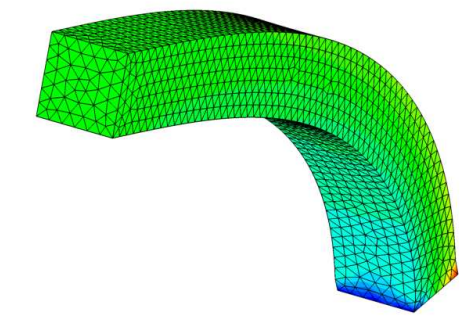

$-227519 \quad-86946.6 \quad 53625.9 \quad 194198$

(a)

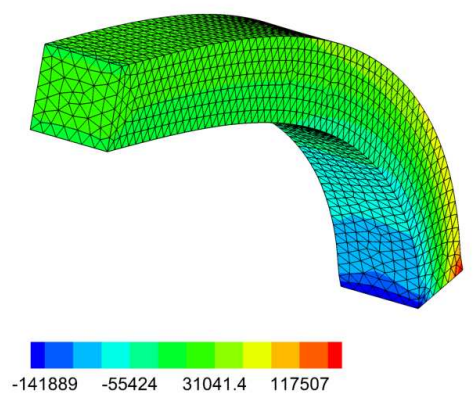

(c)

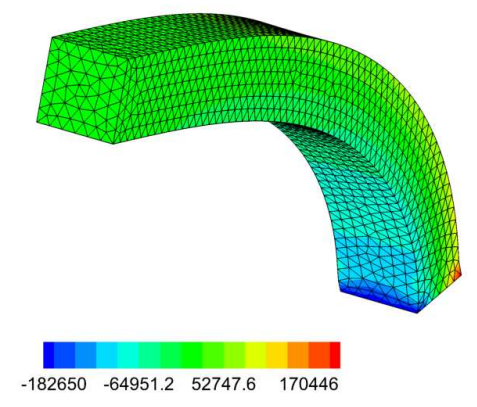

(b)

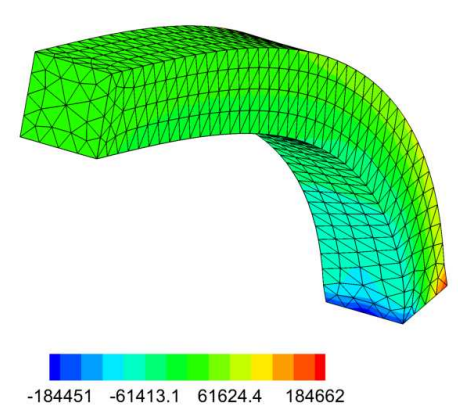

(d)

Figure 12: Contour plot of the hydrostatic pressure distribution $p(k P a)$ for the incompressible long column example. (a) Stabilised P1-P1 formulation with $\tau_{p}=0$ and $\alpha=0.1$. (b) Stabilised P1-P1 formulation with $\tau_{p}=0$ and $\alpha=0.2$. (c) Stabilised P1-P1 formulation with $\tau_{p}=0$ and $\alpha=0.5$. (d) Non-stabilised P2-P0 formulation. Incompresible Mooney-Rivlin model $\hat{W}_{i}$ defined in (176) with material parameters given in (177). Results shown in (a) to (c) are for a discretisation of 17,575 tetrahedral elements $(3,859 \times 3$ degrees of freedom associated to the spatial coordinates $\boldsymbol{x}$ ). Results shown in (d) are for a discretisation of 3,962 tetrahedral elements $(6,675 \times 3$ degrees of freedom associated to the spatial coordinates $\boldsymbol{x}$ ). 


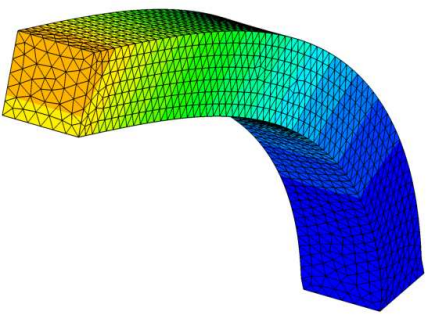

0.51 .52 .53 .54 .55 .56 .57 .58 .5

(a)

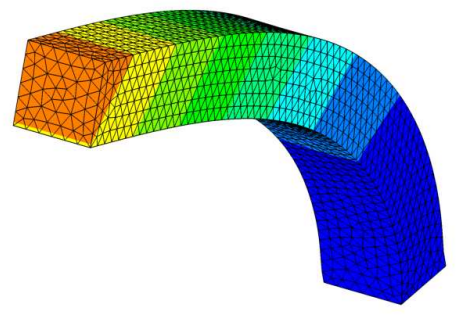

1.02 .03 .04 .05 .06 .07 .08 .0

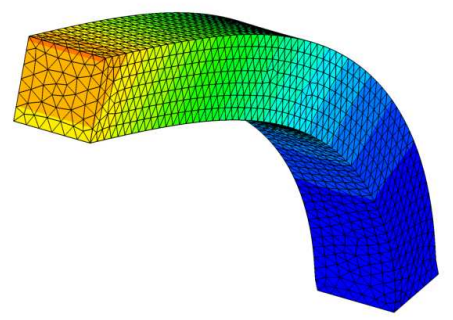

0.51 .52 .53 .54 .55 .56 .57 .58 .5

(b)

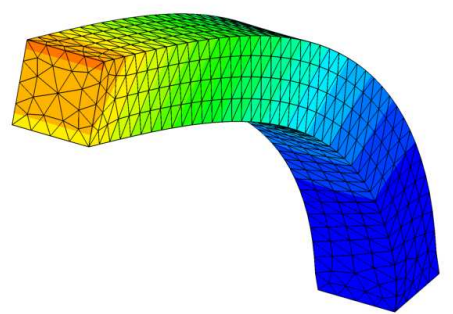

0.51 .52 .53 .54 .55 .56 .57 .58 .5

(d)

Figure 13: Contour plot of the displacement $u_{x}(m)$ distribution for the incompressible long column example. (a) Stabilised P1-P1 formulation with $\tau_{p}=0$ and $\alpha=0.1$. (b) Stabilised P1-P1 formulation with $\tau_{p}=0$ and $\alpha=0.2$. (c) Stabilised P1-P1 formulation with $\tau_{p}=0$ and $\alpha=0.5$. (d) Non-stabilised P2-P0 formulation. Incompresible Mooney-Rivlin model $\hat{W}_{i}$ defined in (176) with material parameters given in (177). Results shown in (a) to (c) are for a discretisation of 17,575 tetrahedral elements $(3,859 \times 3$ degrees of freedom associated to the spatial coordinates $\boldsymbol{x}$ ). Results shown in (d) are for a discretisation of 3,962 tetrahedral elements $(6,675 \times 3$ degrees of freedom associated to the spatial coordinates $\boldsymbol{x}$ ). 


\subsection{Twisting cantilever beam}

The last example includes the twisting of a cantilever beam of length $L=$ $6 m$ and a squared cross sectional area of side $a=1 \mathrm{~m}$. The beam is clamped at its left end and subjected to a torsion on its right end. This example is included to demonstrate the robustness of the mixed formulations. The torsion at the right end is generated through Dirichlet boundary conditions as follows

$$
\left(\boldsymbol{I}-\boldsymbol{E}_{Y} \otimes \boldsymbol{E}_{Y}\right) \boldsymbol{x}=\theta \boldsymbol{E}_{Y} \times \boldsymbol{X}
$$

where $\boldsymbol{E}_{Y}$ is the unit vector normal to the cross section in the reference configuration, $\boldsymbol{X}$ are the initial coordinates, $\theta$ is the angle of rotation and $\boldsymbol{x}$ are the final coordinates. As can be observed, the section is not restricted to in-plane torsion and zero Neumann boundary conditions are imposed normal to the cross sectional area. A similar example has been presented by the authors in previous references $[47,49]$.

The geometry and boundary conditions for the problem are depicted in Figure 14(a). The constitutive model is a compressible Mooney-Rivlin defined by an energy function $W_{u}$ defined by

$$
\begin{aligned}
W_{u}(\boldsymbol{F}, \boldsymbol{H}, J) & =\alpha_{u}(\boldsymbol{F}: \boldsymbol{F})+\beta_{u}(\boldsymbol{H}: \boldsymbol{H})+f_{u}(J) \\
f_{u}(J) & =-4 \beta_{u} \ln J-2 \alpha_{u} \ln J
\end{aligned}
$$

where the material parameters are chosen as

$$
\alpha_{u}=9.0 k P a, \quad \beta_{u}=9.0 k P a .
$$

The selection of these material parameters yield a linear elasticity constitutive operator in the origin defined by shear modulus $\mu=36 k P a$ and Poisson's ratio $\nu=0.25$. The analysis is carried out with a finite element discretisation comprised of 2,304 tetrahedral elements $(4,009 \times 3$ degrees of freedom associated to the spatial coordinates $\boldsymbol{x})$.

Figure 15 displays the contour plot distribution of the stresses $\sigma_{x y}$ (see Figure 15(a) and 15(b)) and $\sigma_{y y}$ (see Figure 15(c) and 15(d)). Results displayed in Figures 15(b) and 15(d) are obtained by using the DF implementation whereas results in Figures 15(a) and 15(c) are obtained by using the alternative mixed formulations, namely $\mathbf{M} 7 \mathbf{F}, \mathbf{M C F}$ and $\mathbf{M} 5 \mathbf{F}$, all yielding identical results. 
As can observed, important differences in terms of stresses can be observed between the $\mathbf{D F}$ formulation and the alternative mixed formulations. This deformation pattern is very demanding for the $\mathbf{D F}$ formulation and improved results would require $h$-refinement. It is important to emphasise that the results are similar in terms of deformation as the problem is displacement driven by the boundary conditions.

The extreme deformation undertaken by the beam leads to a buckled configuration that can be observed in Figure 16, where the out-of-plane configuration can be clearly observed along with the hydrostatic pressure distribution, where negative values (compressive areas) can be identified.

Finally, for completeness, different results concerning some of the components of the kinematic variables $\boldsymbol{F}, \boldsymbol{H}$ and $J$ are shown in Figures 17 and 18. It is possible to observe the smooth distribution of the different fields.

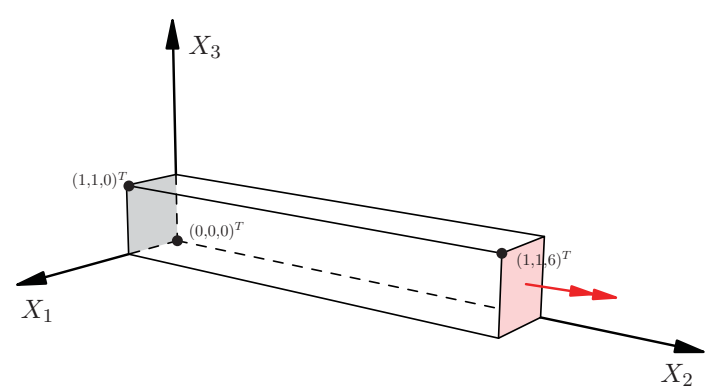

(a)

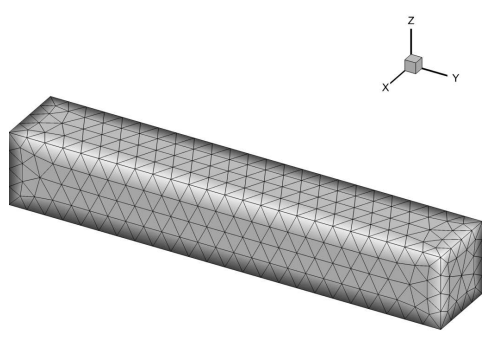

(b)

Figure 14: Twisting cantilever beam problem of length $h=6 m$ and squared cross section defined by its size $a=1 \mathrm{~m}$. (a) Boundary conditions: clamped left end and twisting rotation applied at the right end. Axes $O X_{1}, O X_{2}$ and $O X_{3}$ coincide with ox, oy and $o z$ in (b), respectively. (b) Example of finite element discretisation: 2,304 tetrahedral elements $(4,009 \times 3$ degrees of freedom associated to the spatial coordinates $\boldsymbol{x})$.

\section{Concluding remarks}

This paper has provided a novel approach to formulate large polyconvex large strain elasticity in the computational context. The key novel contributions of the work presented here are: 


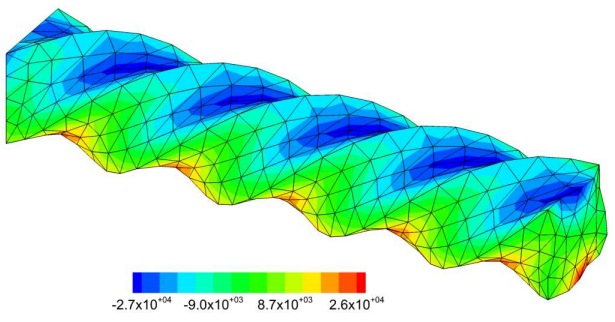

(a)

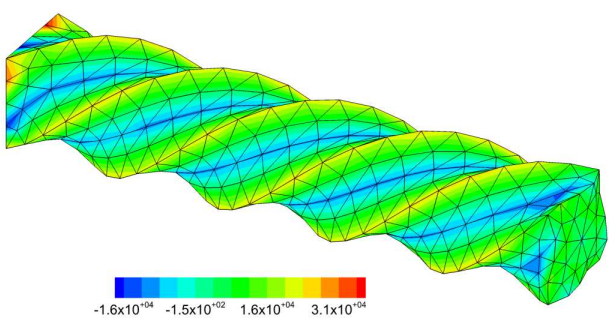

(c)

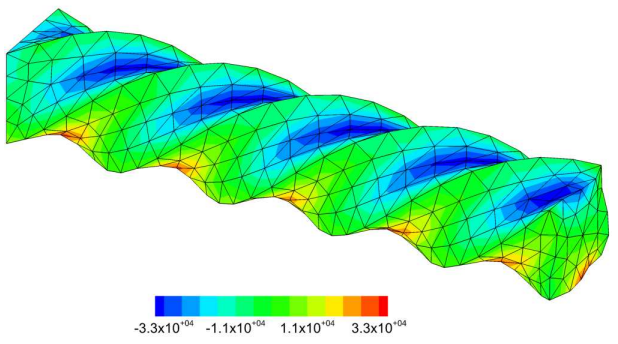

(b)

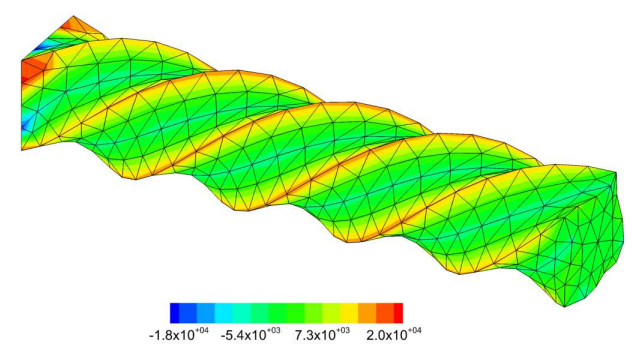

(d)

Figure 15: Contour plot of the stress $\sigma_{x y}(P a)((\mathrm{a})$ and $(\mathrm{b}))$ and $\sigma_{y y}(P a)((\mathrm{c})$ and $(\mathrm{d}))$ distribution for the twisting cantilever beam example. Results obtained with the DF formulation ((b) and (d)) and alternative mixed formulations ((a) and (c)). MooneyRivlin model $W_{u}$ defined in (179) with material parameters given in (180). Results shown for a discretisation of 2,304 tetrahedral elements $(4,009 \times 3$ degrees of freedom associated to the spatial coordinates $\boldsymbol{x}$ ). 

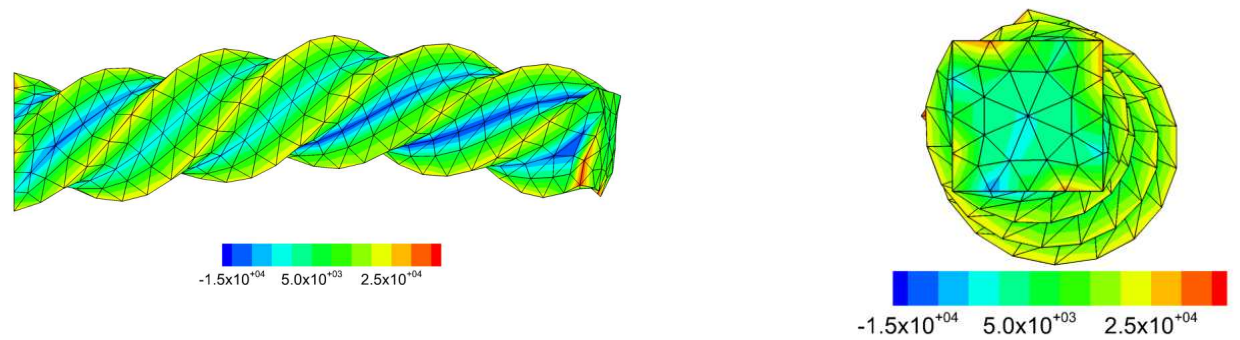

Figure 16: Lateral and plan view of the contour plot of the hydrostatic pressure distribution $p(P a)$ for the twisting cantilever beam example. Results obtained with the alternative mixed formulations. Mooney-Rivlin model $W_{u}$ defined in (179) with material parameters given in (180). Results shown for a discretisation of 2,304 tetrahedral elements $(4,009 \times 3$ degrees of freedom associated to the spatial coordinates $\boldsymbol{x}$ ).

- The definition of a tensor cross product which greatly facilitates the algebraic manipulations of expressions involving the adjoint of the deformation gradient and its derivatives.

- The definition of stresses $\left\{\boldsymbol{\Sigma}_{\boldsymbol{F}}, \boldsymbol{\Sigma}_{\boldsymbol{H}}, \Sigma_{J}\right\}$ conjugate to the main extended kinematic variable set $\{\boldsymbol{F}, \boldsymbol{H}, J\}$ and the development of relationships between these stresses and more classical stress tensors such as the Piola-Kirchhoff stress.

- The definition of a convex complementary strain energy functional in terms of the new set of conjugate stresses $\left\{\boldsymbol{\Sigma}_{\boldsymbol{F}}, \boldsymbol{\Sigma}_{\boldsymbol{H}}, \Sigma_{J}\right\}$.

- The derivation of the elasticity tensor using the simplified algebra facilitated by the tensor cross product definition.

- The formulation of several mixed variational principles including novel Hellinger-Reissner two field versions which reduce the number of problem variables over more traditional three field $\mathrm{Hu}$-Washizu type of principles.

- The introduction of a stabilised Petrov-Galerkin discretisation in the context of mixed principles described above. 


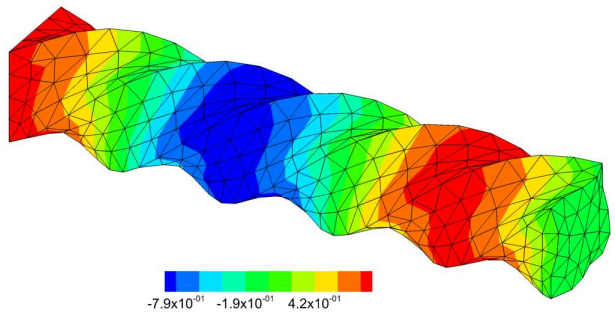

(a)

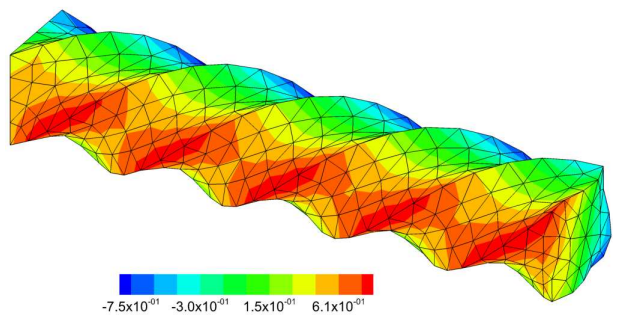

(c)

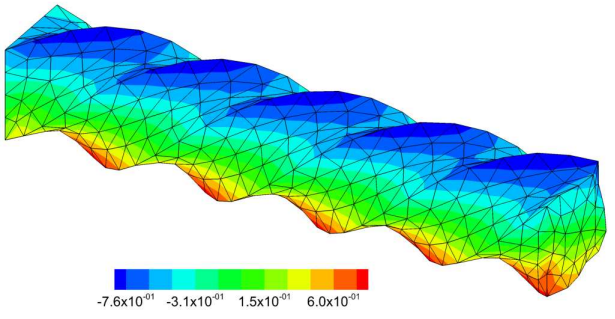

(b)

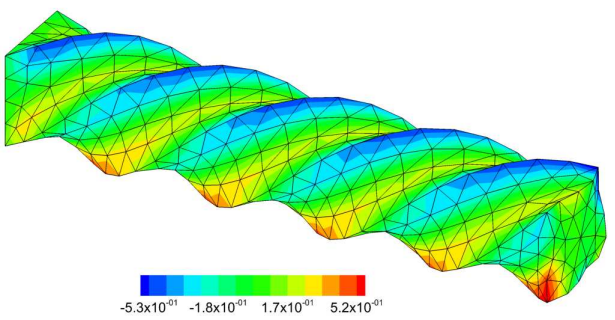

(d)

Figure 17: Contour plot of the distribution of the components (a) $F_{11}$, (b) $F_{12}$, (c) $F_{32}$, (d) $H_{12}$, for the twisting cantilever beam example. Results obtained with the alternative mixed formulations. Mooney-Rivlin model $W_{u}$ defined in (179) with material parameters given in (180). Results shown for a discretisation of 2,304 tetrahedral elements $(4,009 \times 3$ degrees of freedom associated to the spatial coordinates $\boldsymbol{x})$. 


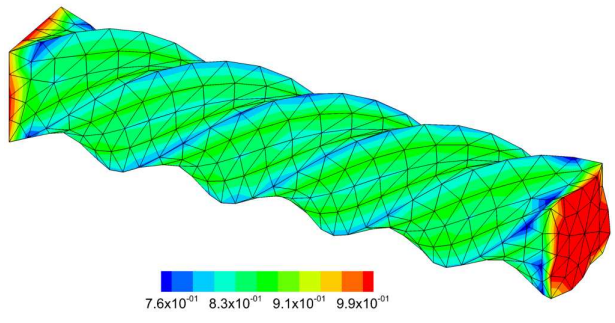

(a)

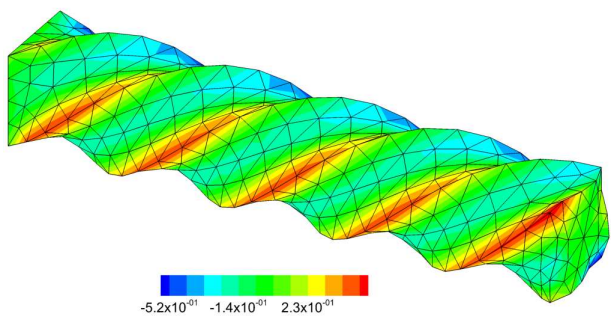

(c)

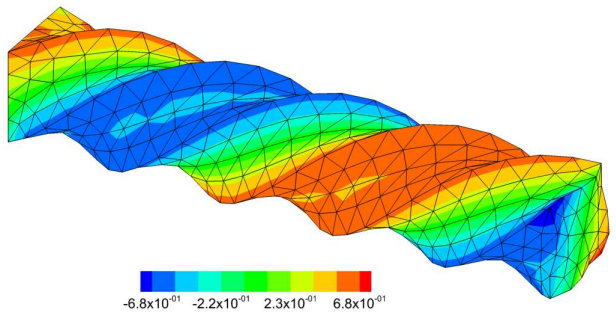

(b)

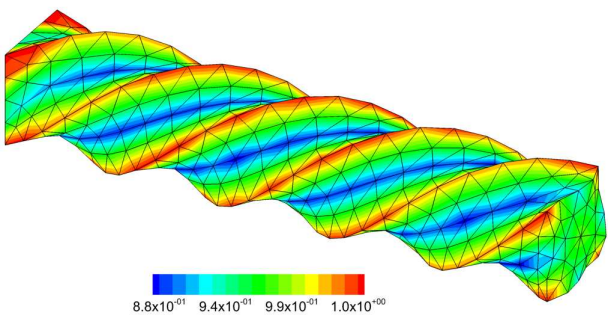

(d)

Figure 18: Contour plot of the distribution of the components (a) $H_{22}$, (b) $H_{21}$, (c) $H_{32}$, and (d) $J$, for the twisting cantilever beam example. Results obtained with the alternative mixed formulations. Mooney-Rivlin model $W_{u}$ defined in (179) with material parameters given in (180). Results shown for a discretisation of 2,304 tetrahedral elements $(4,009 \times 3$ degrees of freedom associated to the spatial coordinates $\boldsymbol{x}$ ). 
The examples provided in the paper have demonstrated that the formulation proposed is capable of modelling complex problems in large strain elasticity and provide order of convergence which is appropriate for the discretisation space chosen. The additional cost involved in the extra variables involved in mixed formulations is significantly reduced in the case of Hellinger-Reissner type of approaches. Moreover, the use of discretisation spaces in which deformation or stress fields are discontinuous across element faces enables these variables to be resolved locally leading to computational cost very comparable to those of displacement based approaches.

Future work will consider the extension of the present framework to solid dynamics using appropriate conservation laws for the extended set of kinematic variables in the manner proposed in references [47, 49-54].

\section{Acknowledgements}

The first author would like to acknowledge the financial support provided by the Sêr Cymru National Research Network for Advanced Engineering and Materials. The second author acknowledges the financial support received through "The Leverhulme Prize" awarded by The Leverhulme Trust, United Kingdom.

\section{References}

[1] J. Bonet, Nonlinear Continuum Mechanics for Finite Element Analysis, 1997.

[2] E. de Souza Neto, D. Períc, D. Owen, Computational Methods for Plasticity. Theory and Applications, 2008.

[3] T. J. R. Hughes, The finite element method: Linear static and dynamic finite element analysis, Dover Publications, 2000.

[4] T. Belytschko, W. K. Liu, B. Moran, Nonlinear finite elements for continua and structures, John Wiley and Sons, 2000.

[5] T. Sussman, K. J. Bathe, A finite element formulation for nonlinear incompressible elastic and inelastic analysis, Computers and Structures 26 (1987) 357-409. 
[6] D. J. Benson, Computational methods in Lagrangian and Eulerian hydrocodes, Computer Methods in Applied Mechanics and Engineering 99 (1992) 235-394.

[7] O. C. Zienkiewicz, J. Rojek, R. L. Taylor, M. Pastor, Triangles and tetrahedra in explicit dynamic codes for solids, International Journal for Numerical Methods in Engineering 43 (1998) 565-583.

[8] M. W. Gee, C. R. Dohrmann, S. W. Key, W. A. Wall, A uniform nodal strain tetrahedron with isochoric stabilization, International Journal for Numerical Methods in Engineering 78 (2009) 429-443.

[9] R. Hill, On uniqueness and stability in the theory of finite elastic strain, Journal of the Mechanics and Physics of Solids 5 (1957) 229-241.

[10] B. D. Coleman, W. Noll, On the thermostatics of continuous media, Archive for Rational Mechanics and Analysis 4 (1959) 97-128.

[11] P. G. Ciarlet, Mathematical Elasticity. Volume 1: Three Dimensional Elasticity, 1988.

[12] O. Gonzalez, A. M. Stuart, A first course in Continuum Mechanics, Cambridge University Press, 2008.

[13] J. E. Marsden, T. J. R. Hughes, Mathematical foundations of elasticity, 1994.

[14] J. M. Ball, Convexity conditions and existence theorems in nonlinear elasticity, Archive for Rational Mechanics and Analysis 63 (1976) 337403.

[15] J. M. Ball, Geometry, Mechanics and Dynamics, Springer, pp. 3-59.

[16] J. M. Ball, F. Murat, W quasiconvexity and variational problems for multiple integrals, Archive for Rational Mechanics and Analysis 63 (1976) 337-403.

[17] J. M. Ball, Energy-minimising configurations in nonlinear elasticity, Archive for Rational Mechanics and Analysis 63 (1976) 337-403. 
[18] K. Zhang, A construction of quasiconvex functions with linear growth at infinity, Annali della Scuola Normale Superiore di Pisa, Classe di Scienze 97 (1992) 313-326.

[19] B. Dacorogna, Direct Methods in the Calculus of Variations, Springer, 2008.

[20] P. Ciarlet, Existence theorems in intrinsic nonlinear elasticity, Journal des mathématiques pures et appliqués 94 (2010) 229-243.

[21] P. N. J. Schröder, Invariant formulation of hyperelastic transverse isotropy based on polyconvex free energy functions, International Journal of Solids and Structures 40 (2003) 401-445.

[22] J. Schröder, P. Neff, V. Ebbing, Anisotropic polyconvex energies on the basis of crystallographic motived structural tensors, Journal of Mechanics and Physics of Solids 56 (2008) 3486-3506.

[23] J. Schröder, P. Neff, V. Ebbing, Polyconvex energies for trigonal, tetragonal and cubic symmetry groups, in: IUTAM-Symposium on variational concepts with applications to the mechanics of materials : Proceedings of the IUTAM-Syposium.

[24] D. B. J. Schröder, P. Wriggers, A new mixed nite element based on different approximations of the minors of deformation tensors, Computational Methods for Applied Mechanical Engineering 200 (2011) 3583-3600.

[25] J. Schröder, Poly-, quasi- and rank-one convexity in Applied Mechanics, CISM Courses and Lectures.

[26] N. Kambouchev, R. Radovitzky, J. Fernández, Anisotropic materials which can be modeled by polyconvex strain energy functions, 47th AIAA/ASME/ASCE/AHS/ASC Structures, Structural Dynamics, and Materials Conference.

[27] K. Washizu, Variational methods in elasticity and plasticity, Pergamon Press, Oxford, 1975.

[28] J. C. Nagtegaal, D. M. Park, J. R. Rice, On numerically accurate finite element solutions in the fully plastic range, Computer Methods in Applied Mechanics and Engineering 4 (1974) 153-177. 
[29] D. S. Malkus, T. J. R. Hughes, Mixed finite element methods - Reduced and selective integration techniques: A unification of concepts, Computer Methods in Applied Mechanics and Engineering 15 (1978) 63-81.

[30] T. J. R. Hughes, Generalization of selective integration procedures to anisotropic and nonlinear media, International Journal for Numerical Methods in Engineering 15 (1980) 1413-1418.

[31] B. Moran, M. Ortiz, C. F. Shih, Formulation of implicit finite element methods for multiplicative finite deformation plasticity, International Journal for Numerical Methods in Engineering 29 (1990) 483-514.

[32] D. P. Flanagan, T. Belytschko, A uniform strain hexahedron and quadrilateral with orthogonal hourglass control, International Journal for Numerical Methods in Engineering 17 (1981) 679-706.

[33] J. Bonet, P. Bhargava, A uniform deformation gradient hexahedron element with artificial hourglass control, International Journal for $\mathrm{Nu}-$ merical Methods in Engineering 38 (1995) 2809-2828.

[34] J. C. Simo, F. Armero, K. S. Pister, Variational and projection methods for the volume constraint in finite deformation elasto-plasticity, Computational Methods for Applied Mechanical Engineering 51 (1985) 177208.

[35] R. Stenberg, A family of mixed nite elements for elasticity problems, Numerische Mathematik 48 (1988) 513-538.

[36] J. C. Simo, F. Armero, Geometrically non-linear enhanced strain mixed methods and the method of incompatible modes, International Journal for Numerical Methods in Engineering 33 (1992) 1413-1449.

[37] J. C. Simo, F. Armero, R. Taylor, Improved versions of assumed enhanced strain tri-linear elements for 3D finite deformation problems, Computational Methods for Applied Mechanical Engineering 110 (1993) 359-386.

[38] M. A. Puso, A highly efcient enhanced assumed strain physically stabilized hexahedral element, International Journal for Numerical Methods in Engineering 49 (2000) 1029-1064. 
[39] F. Auricchio, L. B. de Veiga, C. Lovadina, A. Reali, A stability study of some mixed nite elements for large deformation elasticity problems, Computational Methods for Applied Mechanical Engineering 194 (2005) 1075-1092.

[40] F. Auricchio, L. B. de Veiga, C. Lovadina, A. Reali, The importance of the exact satisfaction of the incompressibility constraint in nonlinear elasticity: mixed fems versus nurbs-based approximations, Computational Methods for Applied Mechanical Engineering 199 (2008) 314-323.

[41] M. Cervera, M. Chiumenti, R. Codina, Mixed stabilized finite element methods in nonlinear solid mechanics: Part I: Formulation, Computer Methods in Applied Mechanics and Engineering 199 (2010) 2559-2570.

[42] R. L. Taylor, A mixed-enhanced formulation for tetrahedral finite elements, International Journal for Numerical Methods in Engineering 47 (2000) 205-227.

[43] C. R. Dohrmann, M. W. Heinstein, J. Jung, S. W. Key, W. R. Witkowski, Node-based uniform strain elements for three-node triangular and four-node tetrahedral meshes, International Journal for Numerical Methods in Engineering 47 (2000) 1549-1568.

[44] Q. Tiehu, Symmetrizing nonlinear elastodynamic system, Journal of Elasticity 50 (1998) 245-252.

[45] D. H. Wagner, Symmetric-hyperbolic equations of motion for a hyperelastic material, Journal of Hyperbolic Differential Equations 06 (2009) 615-630.

[46] A. N. Brooks, T. J. R. Hughes, Streamline upwind/Petrov-Galerkin formulations for convection dominated flows with particular emphasis on the incompressible Navier-Stokes equations, Computer Methods in Applied Mechanics and Engineering 32 (1982) 199-259.

[47] A. J. Gil, C. H. Lee, J. Bonet, M. Aguirre, A stabilised PetrovGalerkin formulation for linear tetrahedral elements in compressible, nearly incompressible and truly incompressible fast dynamics, Computer Methods in Applied Mechanics and Engineering (2014) doi: 10.1016/j.cma.2014.04.006. 
[48] T. J. R. Hughes, L. P. Franca, M. Balestra, A new finite element formulation for computational fluid dynamics: V. Circumventing the Babuška-Brezzi condition: a stable Petrov-Galerkin formulation of the Stokes problem accommodating equal-order interpolations, Computer Methods in Applied Mechanics and Engineering 59 (1986) 85-99.

[49] C. H. Lee, A. J. Gil, J. Bonet, Development of a stabilised PetrovGalerkin formulation for conservation laws in Lagrangian fast solid dynamics, Computer Methods in Applied Mechanics and Engineering 268 (2014) 40-64.

[50] G. Kluth, B. Després, Discretization of hyperelasticity on unstructured mesh with a cell-centered Lagrangian scheme, Journal of Computational Physics 229 (2010) 9092-9118.

[51] C. H. Lee, A. J. Gil, J. Bonet, Development of a cell centred upwind finite volume algorithm for a new conservation law formulation in structural dynamics, Computer Methods in Applied Mechanics and Engineering 118 (2012) 13-38.

[52] N. C. Nguyen, J. Peraire, Hybridizable Discontinuous Galerkin methods for partial differential equations in continuum mechanics, Journal of Computational Physics 231 (2012) 5955-5988.

[53] K. Izian, C. H. Lee, A. J. Gil, J. Bonet, A Two-Step Taylor-Galerkin method for explicit solid dynamics, Engineering Computations 31 (2013) 366-387.

[54] M. Aguirre, A. J. Gil, J. Bonet, A. A. Carreno, A vertex centred finite volume Jameson-Schmidt-Turkel (JST) algorithm for a mixed conservation formulation in solid dynamics, Journal of Computational Physics 259 (2014) 672-699. 